\title{
1 Secondary metabolism drives ecological breadth in the Xylariaceae
}

3 Mario E.E. Franco ${ }^{1}$, Jennifer H. Wisecaver ${ }^{2}$, A. Elizabeth Arnold ${ }^{3}$, Yu-Ming Ju ${ }^{4}$, Jason C. Slot ${ }^{5}$, Steven

4 Ahrendt $^{6}$, Lillian P. Moore ${ }^{1}$, Katharine E. Eastman ${ }^{2}$, Kelsey Scott $^{5}$, Zachary Konkel ${ }^{5}$, Stephen J. Mondo ${ }^{6}$,

$5{\text { Alan } \mathrm{Kuo}^{6} \text {, Richard Hayes }}^{6}$, Sajeet Haridas ${ }^{6}$, Bill Andreopoulos ${ }^{6}$, Robert Riley ${ }^{6}$, Kurt LaButti $^{6}$, Jasmyn

6 Pangilinan $^{6}$, Anna Lipzen ${ }^{6}$, Mojgan Amirebrahimi ${ }^{6}$, Juying $\mathrm{Yan}^{6}$, Catherine Adam ${ }^{6}$, Keykhosrow

7 Keymanesh $^{6}$, Vivian $\mathrm{Ng}^{6}$, Katherine Louie ${ }^{6}$, Trent Northen ${ }^{6}$, Elodie Drula ${ }^{7}$, Bernard Henrissat ${ }^{7}$, Huei-Mei

8 Hsieh $^{4}$, Ken Youens-Clark ${ }^{1}$, François Lutzoni ${ }^{8}$, Jolanta Miadlikowska ${ }^{8}$, Daniel C. Eastwood ${ }^{9}$, Richard C.

9 Hamelin $^{10}$, Igor V. Grigoriev ${ }^{6,11}$, Jana M. U’Ren ${ }^{1 *}$

13 ' ${ }^{1}$ BIO5 Institute and Department of Biosystems Engineering, The University of Arizona, Tucson, AZ 1485721 USA; ${ }^{2}$ Department of Biochemistry, Purdue University, West Lafayette, IN 47907 USA; ${ }^{3}$ School 15 of Plant Sciences and Department of Ecology and Evolutionary Biology, The University of Arizona, 16 Tucson, AZ 85721 USA; ${ }^{4}$ Institute of Plant and Microbial Biology, Academic Sinica, Taipei, Taiwan;

$17{ }^{5}$ Department of Plant Pathology, The Ohio State University, Columbus, OH, 43210 USA; ${ }^{6}$ Department of 18 Energy, The Joint Genome Institute, Lawrence Berkeley National Laboratory, Berkeley, CA 94720 USA;

$19{ }^{7}$ INRAE, USC1408 Architecture et Fonction des Macromolécules Biologiques, 13009, Marseille, France;

$20{ }^{8}$ Department of Biology, Duke University, Durham, NC 27708 USA; ${ }^{9}$ Department of Biosciences,

21 Swansea University, Wales, UK; ${ }^{10}$ Department of Forest and Conservation Sciences, University of British

22 Columbia, Vancouver, British Columbia V6T 1Z4, Canada; ${ }^{11}$ Department of Plant and Microbial

23 Biology, University of California, Berkeley, California 94720 USA.

*Corresponding author: juren@email.arizona.edu, (520)626-0426

Keywords: specialized metabolism, secondary metabolite gene cluster, symbiosis, endophyte, saprotroph, 


\section{ABSTRACT}

36 Global, large-scale surveys of phylogenetically diverse plant and lichen hosts have revealed an extremely

37 high richness of endophytes in the Xylariales, one of the largest clades of filamentous fungi and a

38 significant source of novel secondary metabolites (SMs). Endophytes may produce host protective

39 antimicrobial or insecticidal SMs, as well as compounds that facilitate symbiotic establishment through

40 suppression or degradation of host immune response, but the ecological roles of most SMs are unknown.

41 Here we characterized metabolic gene clusters in 96 genomes of endophytes and closely related

42 saprotrophs and pathogens in two clades of Xylariales (Xylariaceae s.l. and Hypoxylaceae). Hundreds of

43 genes appear horizontally transferred to xylarialean fungi from distantly related fungi and bacteria,

44 including numerous genes in secondary metabolite gene clusters (SMGCs). Although all xylarialean

45 genomes contain hyperabundant SMGCs, we show that increased gene duplications, horizontal gene

46 transfers (HGTs), and SMGC content in Xylariaceae s.l. taxa are linked to greater phylogenetic host

47 breadth, larger biogeographic distributions, and increased capacity for lignocellulose decomposition

48 compared to Hypoxylaceae taxa. Overall, our results suggest that xylarialean endophytes capable of dual

49 ecological modes (symbiotic and saprotrophic) experience greater selection to diversify SMGCs to both

50 increase competitiveness within microbial communities and facilitate diverse symbiotic interactions. 


\section{INTRODUCTION}

Fungal endophytes inhabit asymptomatic, living photosynthetic tissues of all major lineages of plants and lichens to form one of earth's most prevalent groups of symbionts ${ }^{1}$. Known from a wide range of biomes and agroecosystems ${ }^{2,3}$, endophytes are a ubiquitous feature of plant biology ${ }^{4}$. Foliar fungal endophytes are horizontally transmitted, form localized infections, and represent highly diverse and often novel lineages ${ }^{3,5}$. Although classified together due to ecological similar patterns of colonization, transmission, and in planta biodiversity ${ }^{4}$, endophytic fungi represent a diversity of evolutionary histories, life history strategies, and functional traits ${ }^{6}$.

Global, large-scale surveys of phylogenetically diverse plant and lichen hosts have revealed an extremely high richness of endophytes from boreal, temperate, tropical, and subtropical forests in the Xylariales (Sordariomycetes, Pezizomycotina, Ascomycota) ${ }^{7}$, one of the largest clades of filamentous fungi with $>1,300$ named species ${ }^{8}$. Previous multilocus phylogenetic examination of xylarialean endophytes in conjunction with named species (typically found as saprotrophs in decomposing leaves, wood, bark, fruits, or flowers, or more rarely as pathogens in woody hosts) demonstrated that although often closely related to named species, over half of the $\sim 90$ endophyte taxa included in that study appeared as novel, undescribed species ${ }^{7}$. Moreover, the majority of xylarialean endophyte species appear to be host and substrate generalists that associate with multiple lineages of land plants and lichens, as well as in senesced leaves and leaf litter ${ }^{7,9}$.

In addition to their prominence as decomposers and as endophytes in a wide diversity of hosts, xylarialean fungi are a major source of novel metabolic products for use in medicine, agriculture, and industrial biofuel applications ${ }^{10}$. To date, $>500$ SMs have been described from xylarialean fungi, including various cytotoxic, antifungal, and antiparasitic agents ${ }^{10}$. Fungal SMs are often produced by co-

73 localized clusters of genes that are involved in the same metabolic pathway (i.e., SM gene clusters;

74 hereafter SMGCs) ${ }^{11}$. SMGCs typically contain one or more backbone genes (polyketide synthases, non-

75 ribosomal peptide synthetases, hybrid PKS/NRPSs, terpene synthases), as well as accessory genes that

76 modify the molecule through oxidation, reduction, methylation, or glycosylation ${ }^{11}$.

Xylariales genomes sequenced to date have revealed a rich repertoire of SMGCs ${ }^{12}$, often

78 exceeding the numbers reported for fungi well-known for their SM production ${ }^{13,14}$. Such SMs may have

79 various ecological roles: saprotrophic fungi often produce antibiotics and other toxins to inhibit microbial

80 competitors, plant-pathogenic fungi can produce phytotoxic compounds that contribute to virulence ${ }^{15}$, and

81 endophytes can produce antifungals or insect deterrents that protect their hosts ${ }^{16}$. Intense competition with

82 diverse communities of soil organisms is thought to increase selection to maintain and diversify

$83 \mathrm{SMGCs}^{17}$. However, we hypothesized that dual ecological modes of many xylarialean species (symbiotic 
and saprotrophic) ${ }^{7}$ may drive the horizontal gene transfers (HGTs) of SM genes and pathways necessary for both microbial competition and symbiotic establishment within diverse hosts ${ }^{17-20}$.

\section{RESULTS AND DISCUSSION}

Here, we investigated the connections between fungal ecological modes and metabolic gene cluster diversity with 96 genomes within two major clades of Xylariales (Hypoxylaceae and Xylariaceae s.1., hereafter Xylariaceae), including 88 newly sequenced genomes of endophytes, saprotrophs, and plant pathogens (Fig. 1a; Supplementary Fig. 1). Taxa correspond to the previously recognized family Xylariaceae $^{21}$ that was recently split into multiple families (Hypoxylaceae, Graphostromataceae, Barrmaeliaceae $^{22,23}$; Supplementary Table 1). Xylarialean genomes ranged in size from 33.7-60.3 Mbp (average 43.5 Mbp; Supplementary Fig. 2b) and contained ca. 8,000-15,000 predicted genes (average 11,871; Supplementary Fig. 2c), congruent with average genome and proteome sizes of Pezizomycotina ${ }^{24}$. The percentage of repetitive elements per genome ranged from $<1-24 \%$ (average 1.6\%; Supplementary Table 2), but unlike mycorrhizal fungi ${ }^{25}$, repeat content was not corrected with ecological mode (Supplementary Fig. 2d). genomes for SMGCs, as well as a custom pipeline to examine metabolic gene clusters involved in the degradation of a broad array of plant phenylpropanoids ${ }^{27}$ (hereafter, catabolic gene clusters: CGCs).

104 Across 96 xylarialean genomes we predicted a total of 6,879 putative SMGCs (belonging to 3,313 cluster

105 families) and 973 putative CGCs (belonging to 190 cluster families) (Supplementary Tables 3,4). In

106 comparison, recent large-scale analyses predicted 3,399 SMGCs (in 719 cluster families) across 101

107 Dothideomycetes genomes ${ }^{28}$ and 1,110 CGCs across 341 fungal genomes ${ }^{29}$. Only $25 \%$ of predicted

108 SMGCs ( $\mathrm{n}=1,711$, belonging to 816 cluster families) had BLAST hits to 168 unique $\mathrm{MIBiG}^{30}$ accession

109 numbers (Supplementary Table 3b).

110 Total SMGCs diversity in the Xylariaceae and Hypoxylaceae is reflected in a high number of

111 SMGCs per genome: the average number of SMGCs per genome was 71.2 (median 68), which is

112 significantly higher than the average for fungi in the Pezizomycotina (average 42.8; Fig. 1b). At least

113 eight xylarialean genomes contained more than 100 predicted SMGCs, with a maximum of 119 in

114 Anthostoma avocetta NRRL 3190 (Fig. 1b; Supplementary Table 3). In comparison, a recent study of 24

115 species of Penicillium found an average of 54.9 SMGCs per genome, with a maximum number of 78

116 SMGCs observed in P. polonicum ${ }^{13}$. Genomes of Xylariaceae and Hypoxylaceae contained on average 
117 3.3X more CGCs per genome (average 10.1; Supplementary Table 4) compared to genomes of

118 Pezizomycotina (average $3.0^{27}$ ).

119 Every xylarialean genome contained SMGGs for the production of polyketides (PK; 2,871 total),

120 non-ribosomal peptides (NRP; 2,482 total), and terpenes (1,322 total; Fig. 1b; Supplementary Table 3).

121 SMGCs for ribosomally synthesized and post-translationally modified peptides (RiPPs) and hybrid NRP-

122 PK compounds occurred less frequently (Fig. 1b). The most widely distributed and abundant CGCs were

123 pterocarpan hydroxylases $(n=93)$, putatively involved in isoflavonoid metabolism (Fig. 1d,e;

124 Supplementary Table 5). CGCs involved in the breakdown of plant salicylic $\operatorname{acid}^{31}(\mathrm{n}=251$ salicylate

125 hydroxylases) and plant flavonoids ${ }^{27}(\mathrm{n}=170$ naringenin 3-dioxygenases) also were abundant (Fig. 1d,e).

126 CGCs classified into nine other categories (e.g., phenol 2-monooxygenase, quinate dehydrogenase ${ }^{27}$ )

127 occured more rarely (Supplementary Table 4). Vanillyl alcohol oxidases, which were previously shown to

128 be enriched in genomes of soil saprotrophs ${ }^{27}$, were absent in xylarialean genomes.

Consistent with the hyperdiversity of SMGCs in the Hypoxylaceae and Xylariaceae, we observed that only ca. 10\% of SMGCs were shared among genomes from both Xylariaceae and Hypoxylaceae (Fig.

131 1c), and no SMGCs were universally present in both clades (Supplementary Table 3). On average, 21.4\%

132 and $28.2 \%$ of SMGCs per genome were unique to either taxa in the Hypoxylaceae or the Xylariaceae,

133 respectively (range 0-82\%; Fig. 1c; Supplementary Table 4), but no SMGCs were universally present

134 within either clade. For most isolates, the majority of SMGCs were unique (i.e., 'isolate specific'; Fig. 1c).

135 Isolate specific SMGCs represented an average of $36.6 \%(\mathrm{SD} \pm 21.1)$ of the clusters per genome (range 0-

$13685.7 \%$; Fig. 1c). Even when multiple isolates of the same species were compared (e.g., Nemania serpens

137 clade) $30-41 \%$ of the SMGCs appeared specific to a single isolate (Fig 1b; see also Supplementary Table

138 3), similar to intraspecific SMGC variation in Aspergillus flavus ${ }^{14}$.

140 Impact of HGT on xylarialean genome evolution. To assess the role of HGT in shaping the genome

141 evolution of Xylariaceae and Hypoxylaceae we performed two Alien Index (AI) analyses ${ }^{32-34}$. The first

142 AI screen - designed to detect candidate HGTs from more distantly related donor lineages (e.g., bacteria,

143 plants) —flagged 4,262 genes representing 647 orthogroups (Supplementary Table 5a). Using a custom

144 phylogenetic pipeline (see Methods) we then identified 168 potential HGT events to Xylariaceae and

145 Hypoxylaceae. Based on branch support and the presence of multiple xylarialean taxa in the recipient

146 clade, 92 of these genes were deemed high-confidence HGTs (Fig. 2; Supplementary Table 5b). Similar

147 to previous studies ${ }^{35,36}$, the majority of high-confidence HGTs are predicted to have been acquired from

148 bacteria $(n=86)($ Fig. 2). Other donor lineages include viruses $(n=3)$, Basidiomycota $(n=2)$ and plants

149 ( $\mathrm{n}=1)$ (Fig. 2; Supplementary Table 5b). On average, xylarialean genomes had 16.2 high-confidence 
HGT events per genome (range: 7-30; Supplementary Table 5c). The highest number of high-confidence HGT events per genome occurred in the genome of Xylaria flabelliformis CBS $123580(\mathrm{n}=30)$.

HGT candidate genes were typically distributed across taxa in numerous diverse clades $(n=85$ of 92 genes) rather than in monophyletic clades (Fig. 2). For example, an Enoyl-acyl carrier protein

154 reductase protein (EC 1.3.1.9) - a key enzyme of the type II fatty acid synthesis (FAS) system ${ }^{37}$ -

155 occurred in bacteria (putative donor) and four distantly related recipient taxa: Xylariales sp. PMI 506,

156 Hypoxylon rubiginosum ER1909; H. cercidicola CBS 119009; H. fuscum CBS 119018 (HGT0001;

157 Supplementary Table 5). Multiple evolutionary scenarios could result in patchy taxonomic distributions.

158 For example, multiple fungi could have independently acquired the same gene from closely related

159 bacterial donors ${ }^{36}$. Alternatively, an initial HGT from bacteria to fungi may have been followed by

160 fungal-fungal HGTs. In total, 38 HGT candidate genes occurred in genomes of both Sordariomycetes

161 outgroup and Xylariales genomes, 28 were found in only Xylariales genomes, and 26 were only observed

162 in genomes of Xylariaceae and Hypoxylaceae (Fig. 2; Supplementary Table 5b).

Functional annotation revealed the majority of candidate HGT genes were associated with at least one type of annotation (i.e., $95 \%$ of the highly confident and $82 \%$ of the ambiguous events; $43 \%$ of candidate HGT genes were predicted to be part of a SMGC (i.e., 40 of 92) (Fig. 2; Supplementary Tables 3,5). These include 13 genes predicted to have a biosynthetic function, such as a putative FsCacetyl coenzyme A-N²-transacetylase (HGT076; Supplementary Table 5), which is part of the siderophore

172 biosynthetic pathway in Aspergillus implicated in fungal virulence ${ }^{38}$.

173 Due to the high prevalence of HGT among genes predicted to be part of SMGCs, we performed a

174 second AI screen to detect intra-fungal HGT events of genes within the boundaries of SMGCs $(\mathrm{n}=$

175 93,066 genes) (see Methods; Supplementary Fig. 3). This analysis identified 1,148 genes in 660 SMGCs

176 (belonging to 594 cluster families) that were putatively transferred from other fungi to members of the

177 Xylariales (Supplementary Table 5). Candidate HGT genes were primarily for polyketide and non-

178 ribosomal peptide production (518 PKSs, 270 NRPSs, and 180 PKS-NRPS hybrid clusters). In addition,

$179>75 \%$ of hits to MIBiG contain genes identified by AI analyses as putative HGTs (127 of 168; see Fig. 3,

180 bottom). SMGCs with HGT candidate genes include those with $100 \%$ similarity to MIBiG accessions

181 from Aspergillus, Fusarium, and Parastagonospora involved in mycotoxin (e.g., cyclopiazonic acid,

182 alternariol, fusarin) and antimicrobial compound (asperlactone, koraiol) production, and clusters from

183 Alternaria that produce host-selective toxins (e.g., ACT-Toxin II) (Supplementary Tables 3,5). Although 
184 the second AI analysis did not identify each gene in these clusters as potential HGTs (e.g., 4 of the 19

185 genes in the alternariol cluster from Hypoxylon cercidicola CBS 119009 were predicted to be HGT;

186 Supplementary Table 5), the phylogenetic distribution of many of these SMGCs is consistent with the

187 acquisition of SMGCs via HGT (Fig. 3).

188 Within this phylogenomic framework we also identified additional SMGCs with high similarity

189 (i.e., calculated as the percentage of genes in an SMGC with significant BLAST hits to a known SMGC ${ }^{39}$ )

190 to fungal MIBiG accessions and phylogenetic distributions that support putative HGT to Xylariaceae and

191 Hypoxylaceae (Fig. 3), but were not flagged by the second AI analysis. For example, xylarialean SMGCs

192 with $>70 \%$ similarity to clusters for ergoline alkaloids and their precursors (e.g., loline, ergovaline, and

193 lysergic acid production) produced by Clavicipitaceae endophytes, as well as the phytotoxins cichorine

194 cluster from Aspergillus (Fig. 3; Supplementary Table 3). The griseofulvin cluster from Penicillium

195 aethiopicum, which produces a potent antifungal compound ${ }^{40}$, also appears horizontally transferred to the

196 clade containing X. castorea and X. flabelliformis isolates (Fig. 3; Supplementary Fig. 4). Our analyses of

197 HGT provide the highest support for HGTs from distantly related hosts such as bacteria (Fig. 2; see also

$198{ }^{36}$ ), but our data also support fungal-fungal HGT as an important mechanism of metabolic innovation in

199 the Xylariales. Although the discontinuous phylogenetic distributions of SMGCs observed here may

200 represent unequal gene loss across taxa ${ }^{11,17}$, the presence of entire clusters known from Eurotiomycetes

201 and Sordariomycetes in multiple endophytic and non-endophytic taxa provides additional support for

202 HGTs.

Expansion of Xylariaceae genomes due to increased gene duplication and HGTs. Despite the close

205 evolutionary relationship and similar ecological niches of taxa in the Xylariaceae and Hypoxylaceae, 206 genomes of Xylariaceae were on average ca. 7.2 Mbp larger than genomes of Hypoxylaceae (Fig. 4a;

207 Supplementary Table 6). Larger genome size was associated with higher repeat content: Xylariaceae

208 contained an average of 2-fold more repetitive elements (Fig. 4b; Supplementary Table 6) and had a

209 higher density of repetitive elements surrounding genes compared to Hypoxylaceae genomes

210 (Supplementary Fig. 5).

211 In addition to greater repeat content, Xylariaceae genomes also contained on average 750 more

212 protein-coding genes compared to Hypoxylaceae genomes $(\mathrm{P}<0.0001$; Supplementary Table 6b).

213 Ancestral state reconstructions reveal that Xylariaceae genomes have experienced significantly more gene

214 gains $(n=472)$, gene duplication events $(n=136)$, orthogroup gains $(n=313)$, and orthogroup expansion

215 events $(\mathrm{n}=90)$ compared to Hypoxylaceae clade since the radiation from their last common ancestor

216 (Fig. 4c-d), although both clades underwent similar numbers of gene losses $\left(\mathrm{t}_{95}=0.51, \mathrm{P}=0.61\right.$; 
217 Supplementary Table 6b). Xylariaceae genomes also experienced on average ca. 2-fold more HGTs

218 events compared to Hypoxylaceae genomes (Fig. 4e).

219 Increased genome sizes resulting from HGT were positively associated with increased numbers of

220 SMGCs across both clades (Fig. 4f), reflecting the fact that clustered metabolite genes in fungi are more

221 likely to undergo HGT compared to unclustered genes ${ }^{41}$. Genomes of Xylariaceae contained on average

222 ca. 20 more SMGCs than Hypoxylaceae genomes (Supplementary Table 6b) and ca. 2-fold greater

223 cumulative richness of SMGCs compared to Hypoxylaceae clade (2,336 vs 1,075 total; 587 vs 282 non-

224 singleton). Rarefaction analysis reveals the richness of non-singleton SMGCs increases at a greater rate in

225 the Xylariaceae clade (Fig. 4g). Genomes of Xylariaceae also contained a greater fraction of isolate

226 specific SMGCs compared to Hypoxylaceae, regardless of SMGC type (Xylariaceae: $31.2 \pm 16.1$;

227 Hypoxylaceae: $19.8 \pm 15.3$; P = 0.0007; Fig. 1c; Supplementary Fig. 6). Yet despite the high variation of

228 SMGCs among taxa, network analysis illustrates that the composition of SMGCs is more similar among

229 isolates from the same clade, regardless of ecological mode (Supplementary Fig. 7).

230 In contrast to the pattern observed for SMGCs, genomes of Hypoxylaceae contained a greater

231 number of CGCs than Xylariaceae genomes (Xylariaceae: $9.5 \pm 0.4$; Hypoxylaceae: $11.0 \pm 0.4 ; \mathrm{P}=$

232 0.0068; Supplementary Table 4) and different classes of CGC dominated the two clades (Fig. 1d,e). For

233 example, salicylate hydroxylases were the most abundant CGCs among Hypoxylaceae, but were absent

234 from 25\% Xylariaceae genomes (Fig. 1d). In contrast, CGCs classified as pterocarpan hydroxylases were

235 the most abundant CGC type in genomes of Xylariaceae (Fig. 1d). Four types of CGCs were universally

236 present across Hypoxylaceae: salicylate hydroxylase, pterocarpan hydroxylase, naringenin 3-dioxygenase,

237 phenol 2-monooxygenase (Fig. 1d). CGCs classified as naringenin 3-dioxygenase were the only CGC

238 type found across all Xylariaceae genomes.

239 In addition to different metabolic gene clusters content, additional differences between

240 Xylariaceae and Hypoxylaceae genomes suggest different functional capacities. Xylariaceae genomes

241 contain greater numbers of genes with signaling peptides, as well as genes annotated as effectors,

242 membrane transport proteins, transcription factors, peptidases, and CAZymes compared to Hypoxylaceae,

243 even after accounting for differences in genome size (Supplementary Table 6). For example, on average

244 genomes of Xylariaceae contained ca. 50 more CAZymes than Hypoxylaceae (Xylariaceae 579.9 \pm 7.7 ;

245 Hypoxylaceae 529.6 $\pm 9.1, \mathrm{P}<0.0001$ ), including a significant increase in PCWDEs involved in the

246 degradation of cellulose, hemicellulose, lignin, pectin, and starch (Supplementary Table 6). Additionally,

247 comparison of gene ontology (GO) terms for shared orthogroups significantly enriched in either

248 Xylariaceae or Hypoxylaceae (i.e., 74 and 26, respectively) revealed that the Hypoxylaceae had a

249 significant increase in the number of GO terms associated with membrane transport, whereas Xylariaceae 
had a significant increase in the number of GO terms for catalytic activities and binding (Supplementary Fig. 8b).

Xylariaceae genome evolution linked to ecological generalism. The majority of described Xylariaceae and Hypoxylaceae species are wood- or litter-degrading saprotrophs or woody pathogens ${ }^{42,43}$, although both culture-based and culture-free studies of healthy photosynthetic tissues of plants and lichens demonstrate the abundance and novel diversity represented by xylarialean endophytes ${ }^{7}$. Previous studies have identified isolates with highly similar ITS nrDNA sequences occurring in both living host tissues as well as decomposing plant materials ${ }^{7,9}$, which suggests that endophytism may represent only part of a complex life cycle that blurs the lines between distinct ecological modes ${ }^{7}$.

In support of such ecological generalism, we observed no clear distinctions in genome size or content among endophytic and non-endophytic taxa when all ingroup genomes were analyzed (Supplementary Table 6). One exception was the reduced genomes and CAZyme content of termiteassociated Xylaria spp. (i.e., X. nigripes YMJ 653, X. sp. CBS 124048, and X. intraflava YMJ725; specialization on termite nest substrates decomposed by a basidiomycete fungus ${ }^{43}$. The lack of clear genomic signal for endophytism in the Xylariaceae and Hypoxylaceae contrasts sharply with genome evolution in ectomycorrhizal fungi, where mycorrhizal clades have experienced convergent loss of genes that encode lignocellulose-degrading enzymes and an increase in small secreted effector-like proteins since their divergence from saprotrophic ancestors ${ }^{25}$. However, a recent analysis of 101 ecologically diverse Dothideomycetes revealed only six orthogroups predicted plant-pathogenic vs. saprotrophic ecological mode with $>95 \%$ accuracy $^{44}$, highlighting the complexity of linking genotype to phenotype for complex traits.

Despite their ecological similarities, genomes of Xylariaceae experienced more gene duplications,

274 gene family expansions, and HGT events, resulting in higher SMGC content as well as more genes

275 important for pathogenicity (e.g., effectors, peptidases) and saprotrophy (e.g, CAZymes, transporters) in

276 comparison with Hypoxylaceae (Supplementary Table 6). As genomes of fungi with saprotrophic

277 lifestyles typically contain more CAZymes and PCWDEs compared to plant pathogens and mycorrhizal

278 symbionts ${ }^{25,44,45}$, our genomic results are consistent with the potential for Xylariaceae fungi (including

279 endophytes) to have greater saprotrophic abilities compared to Hypoxylaceae fungi ${ }^{46}$. To test this

280 prediction, we compared the abilities of 20 isolates to degrade leaves of Pinus and Quercus. We found

281 that isolates of Xylariaceae with expanded CAZymes and PCWDEs repertoires caused greater mass loss

282 compared to taxa with fewer genes predicted to degrade lignocellulose (i.e., Hypoxylaceae and

283 Xylariaceae from animal-dung clade; Supplementary Fig. 10). 
The genomic and functional differences we observed are consistent with Xylariaceae species as ecological generalists encompassing both endophytic and saprotrophic life stages. Xylariaceae endophyte species also associate with a greater phylogenetic diversity of plant and lichen hosts compared to species of Hypoxylaceae endophytes ( $\mathrm{t}_{42}=2.25 ; \mathrm{P}=0.0294$; Supplementary Fig. 11a). Host breadth of Xylariaceae endophytes also is positively associated with the number of total HGT events and the number of SMGCs for non-ribosomal peptides (Supplementary Fig. 11b). Thus, we hypothesized Hypoxylaceae taxa may have undergone fewer HGT events and gene expansions due to species having more distinct ecological modes with less selection for metabolic versatility. To test this hypothesis, we performed

292 pairwise comparisons of 15 sister taxa across both clades with contrasting ecological modes, which 293 revealed that endophyte genomes in the Hypoxylaceae contain significantly fewer genes with signaling

294 peptides, protein coding genes, transporters, peptidases, PCWDEs (especially those involved in 295 decomposition of cellulose and lignin), SMGCs, and CGCs than non-endophyte genomes (Fig. 5). In

296 contrast, no significant differences were observed between endophytes and saprotrophs in the Xylariaceae clade (Fig. 5; Supplementary Table 6).

Increased metabolic diversity and host breadth of Xylariaceae species likely impacts their geographical distributions ${ }^{47}$. Xylariaceae genera such as Xylaria and Nemania occur worldwide as fruiting

300 bodies in temperate, subtropical, and tropical forests, whereas Hypoxylaceae genera such as Daldinia and

301 Hypoxylon are more common in boreal and temperate forests ${ }^{7}$, but taxonomic uncertainty for many

302 specimens and sequences ${ }^{48}$ combined with a lack of biome metadata for the majority of reference taxa $^{7}$

303 precludes robust statistical comparisons of biogeographic ranges for named taxa. However, a recent

304 global survey of boreal endophytes demonstrated that host generalist species occupy larger geographic

305 ranges $^{3}$ and re-analysis of data from previous ecological surveys in boreal, temperate montane, and

306 subtropical forests in Alaska, Arizona, North Carolina, and Florida reveals a higher fraction of

307 Xylariaceae endophyte species cultured from hosts in more than one site (i.e., 28\% Xylariaceae vs. 20\%

308 for Hypoxylaceae), including six Xylariaceae endophyte species that were found in $>3$ sites $^{7}$. In contrast, 309 no Hypoxylaceae endophyte species from that study were found in more than two sites ${ }^{7}$.

\section{CONCLUSIONS}

312 Our analysis of 96 phylogenetically and ecologically diverse Xylariaceae and Hypoxylaceae genomes

313 reveals that gene duplication, gene family expansion, and HGT of SMGCs from putative bacterial and

314 fungal donors, drives metabolic versatility in the Xylariaceae. Expanded metabolic diversity of

315 Xylariaceae taxa is associated with greater phylogenetic host breadth, larger biogeographic distributions, 316 and increased capacity for lignocellulose decomposition compared to Hypoxylaceae taxa. Yet despite 317 differences among clades, our data suggest that saprotrophs in both clades are under selection to maintain 
318 both large gene repertoires to degrade diverse lignocellulosic compounds ${ }^{44}$ and highly diverse SMGCs

319 that likely increase competitive abilities in diverse microbial communities ${ }^{11,49,50}$ (Supplementary Table

320 6e). In contrast, SMGC abundance in endophyte genomes appears unrelated to PCWDE content. Overall,

321 our results provide evidence that SMGCs may play a key role in facilitating endophyte colonization of

322 diverse hosts (e.g., through suppression or degradation of host immune responses ${ }^{17,18}$ ), further

323 highlighting the importance of symbioses to drive not only speciation and ecological diversification ${ }^{51}$, but

324 chemical biodiversity that can be leveraged for novel pharmaceuticals and agrochemicals ${ }^{52}$.

\section{METHODS}

327 Fungal strain selection and verification. Isolates were selected based on their phylogenetic position

328 (previously estimated using multilocus phylogenetic analyses ${ }^{7}$ ), as well as their ecological mode (i.e.,

329 endophyte, saprotroph, pathogen). To minimize the effect of phylogeny when assessing the impact of

330 ecological mode on genome evolution, we included 15 pairs of closely related sister taxa with contrasting

331 ecological modes (i.e., endophyte vs. non-endophyte) ${ }^{7}$. Ecological modes were assigned based on the

332 substrate of isolation: fungi isolated from living plants and lichens with no signs of disease were

333 classified as endophytic; fungi isolated from or collected as fruiting bodies from decomposing plant

334 tissues (e.g., litter, wood, dung) were classified as saprotrophs; and fungi isolated or collected as fruiting

335 bodies from living, diseased host tissues were classified as pathogens. For strains that lacked host and

336 substrate metadata, ecological modes were estimated based on information for that species in the

337 literature (see $\operatorname{ref}^{7}$ ).

338 In total, we sequenced 44 endophytic taxa ${ }^{2,53}$ and 44 named taxa of Xylariaceae s.1. and

339 Hypoxylaceae (Supplementary Table 1). Endophytic isolates are maintained as an axenic voucher in

340 sterile water at the Robert L. Gilbertson Mycological Herbarium at the University of Arizona (ARIZ).

341 Cultures of named taxa were obtained from the Westerdijk Fungal Biodiversity Institute (Netherlands) or

342 from Dr. Yu-Ming Ju. In total, we sequenced genomes representing ca. 24 genera and 80 species of

343 Xylariaceae s.l. and Hypoxylaceae, as well as an additional two undescribed species of endophytic

344 Xylariales (Pestalotiopsis sp. NC0098 and Xylariales sp. AK1849) included in the outgroup

345 (Supplementary Fig. 1).

346 Prior to genome and transcriptome sequencing, fungi were grown on 2\% malt extract agar (MEA)

347 to verify morphology and obtain tissue for a preliminary DNA extraction to verify isolate identity.

348 Briefly, DNA was extracted using Extract n Amp (Sigma) following ref ${ }^{54}$. For each isolate the ITS-LSU

349 nrDNA region was PCR amplified using the primer pair ITS1F/LR3 and Sanger sequenced for each

350 isolate as described by ref $^{2}$. Sequences were edited in Sequencher v5.4.6 (Gene Codes Corporation, Ann

351 Arbor, MI) and aligned with the original ITS nrDNA sequences for each isolate. For isolates without a 
352 prior ITS nrDNA sequence, we used T-BAS v2 ${ }^{55}$ to query sequences against the multilocus tree of the

353 Xylariaceae from $\operatorname{ref}^{7}$. In some cases, names of reference taxa (previously named based only on

354 morphological characters) were updated to reflect their phylogenetic placement (see Supplementary Table

$3551)$.

DNA and RNA purification. After strains were verified, we used two different mycelial growth and cultivation techniques to achieve the specific nucleic acid concentration and quality requirement for either Illumina or PacBio Single-Molecule Real-Time (SMRT) sequencing. For PacBio sequencing, isolates were first grown on multiple 2\% MEA plates overlaid with sterile, cellophane membrane to allow mycelial harvesting without media carry-over. After ca. 5-10 days of growth, mycelium was removed using sterile forceps and scalpels, placed in $150 \mathrm{~mL}$ of $1 \%$ malt extract (ME) media in a sterile, stainless steel Eberbach blender cup (Fisher Scientific) and homogenized with 3-5 short pulses using a Waring blender. After homogenization, two $75 \mathrm{~mL}$ aliquots were placed in Erlenmeyer flasks and incubated on a shaker at room temperature for 3-7 days. Once sufficient growth was obtained samples were then filtered through sterilized Miracloth (Millipore, 475855-1R) in a Buchner funnel (Fisher Scientific), placed in a $50 \mathrm{~mL}$ centrifuge tube, flash-frozen in liquid nitrogen, and stored at $-80^{\circ} \mathrm{C}$. If isolates grew slowly, the contents of the inoculated flask were re-blended with an equal volume of fresh 1\% ME media after 7 days, aliquoted into new flasks, and incubated on the shaker at room temperature for an additional 5-7 days prior to filtering. After filtration, mycelium was washed with sterile molecular grade water to remove media and excess polysaccharides.

DNA isolation for PacBio sequencing was performed using a modified phenol:chloroform extraction method (see ref ${ }^{56}$ ). Briefly, ca. $4 \mathrm{~g}$ (wet weight) of tissue was ground in liquid nitrogen with a sterile mortar and pestle. Ground tissue was transferred to a $50 \mathrm{~mL}$ Falcon tube containing $14 \mathrm{~mL}$ of SDS buffer and incubated at $65^{\circ} \mathrm{C}$ for 30 minutes, during which the tube was gently inverted $5 \mathrm{X}$ every 10 minutes. After incubation, $0.5 \mathrm{X}$ volume of $5 \mathrm{M} \mathrm{KOAc}(\mathrm{pH} 7.5)$ was added to each tube, mixed by inversion, and placed at $4^{\circ} \mathrm{C}$ for 30 minutes. Samples were then centrifuged at 4500 RPM for 10 minutes at $4^{\circ} \mathrm{C}$. After centrifugation, the supernatant was removed, placed into a new tube, $0.7 \mathrm{X}$ volume of molecular grade isopropanol was added, and the tube was gently inverted to mix. The sample was then centrifuged at $4500 \mathrm{RPM}$ for 20 minutes at $4^{\circ} \mathrm{C}$ to precipitate the DNA. After centrifugation the supernatant was removed, and the DNA pellet was washed with $5 \mathrm{~mL}$ of $70 \% \mathrm{EtOH}$ and centrifuged for an additional 5 minutes at 4500 RPM. Residual EtOH was removed with a pipette, and the pellet was air 383 dried. The DNA pellet was resuspended in $2 \mathrm{~mL}$ of TE buffer, $10 \mathrm{uL}$ of RNase $(20 \mathrm{mg} / \mathrm{mL}$; Invitrogen, 384 Waltham, MA) and the sample was placed in a $37^{\circ} \mathrm{C}$ water bath for 1 hour. After incubation, DNA was 385 purified with phenol:chloroform:IAA, washed with $0.3 \mathrm{X}$ volume of absolute molecular grade ethanol to 
remove polysaccharides, and precipitated by adding $1.7 \mathrm{X}$ volume of absolute molecular grade ethanol. The resulting DNA pellet was washed with $70 \% \mathrm{EtOH}$, air dried, and resuspended in low salt TE.

For Illumina sequencing, isolates were first grown on multiple 2\% MEA plates overlaid with sterile cellophane as described above, but harvested mycelium was placed in RNase free stainless-steel bead tubes (Next Advance, NAVYR5-RNA), flash frozen in liquid nitrogen, and stored at $-80^{\circ} \mathrm{C}$ until extraction. DNA for Illumina sequencing was extracted using similar methods as above for PacBio, with the exception that only a small amount of tissue was used, samples were homogenized in $2 \mathrm{~mL}$ tubes with stainless steel beads rather than grinding in liquid $\mathrm{N}$, and the initial purification with $5 \mathrm{M}$ KOAc was not performed (see ref ${ }^{57}$ ). DNA obtained from both methods was quantified with a Qubit fluorometer (Invitrogen, Carlsbad, CA) and sample purity was assessed with a NanoDrop 1000 (BioNordika, Herlev, Denmark). The purity of DNA for PacBio sequencing was also verified with a EcoRI (New England BioLabs, Ipswich, MA) restriction digest and sized via electrophoresis on a $1 \%$ agarose gel with a clamped homogeneous electric field (CHEF) apparatus ${ }^{58}$ as described in ref ${ }^{59}$.

RNA was extracted for each isolate with the Ambion Purelink RNA Kit (Thermo Fisher Scientific, Waltham, MA). Briefly, isolates were grown on 2\% MEA with sterile cellophane overlay. Mycelium was harvested after ca. one week of growth, placed in $2 \mathrm{~mL}$ tubes containing stainless steel beads, flash frozen in liquid $\mathrm{N}$, and stored at $-80^{\circ} \mathrm{C}$ until extraction. Frozen mycelium was homogenized for 5 seconds at 1400 RPM on a BioSpec, Mini-BeadBeater 96 115V (MP Biomedicals) with stainless steel beads. Following homogenization, $1 \mathrm{~mL}$ of TRIzol was added to each tube and the sample was incubated for 5 minutes at room temperature, followed by centrifugation at $4^{\circ} \mathrm{C}$ for 15 minutes at 12,000 RPM. Following centrifugation, the supernatant was transferred to a new tube and $0.2 \mathrm{~mL}$ of chloroform was added, mixed gently by inversion, and transferred to a column following the manufacturer's instructions. RNA was quantified with a Qubit fluorometer (Invitrogen) and sample purity was assessed with a NanoDrop (BioNordika). RNA was then treated with DNase (Thermo Fisher Scientific) following the manufacturer's instructions and RNA integrity was assessed on a BioAnalyzer at the University of Arizona Genomics Core Facility.

Genome and transcriptome sequencing and assembly. Genomes were generated the Department of

414 Energy (DOE) Joint Genome Institute (JGI) using Illumina and PacBio technologies (Supplementary

415 Table 1). For 66 isolates, Illumina standard shotgun libraries (insert sizes of 300bp or 600bp) were

416 constructed and sequenced using the NovaSeq platform. Raw reads were filtered for artifact/process

417 contamination using the JGI QC pipeline. An assembly of the target genome was generated using the

418 resulting non-organelle reads with SPAdes ${ }^{60}$. PacBio SMRT sequencing was performed for 22 isolates of

419 Xylariaceae s.l. and Hypoxylaceae and two additional endophytic Xylariales (Xylariales spp. NC0098 and 
AK1849) on a PacBio Sequel. Library preparation was performed either using the PacBio Low Input $10 \mathrm{~kb}$ or PacBio $>10 \mathrm{~kb}$ with AMPure Bead Size Selection. Filtered sub-read data were processed with the JGI QC pipeline and de novo assembled using Falcon (SEQUEL) or Flye (SEQUEL II). Stranded RNASeq libraries were created and quantified by qPCR. Transcriptome sequencing was performed on an Illumina NovaSeq S4. Raw reads were filtered and trimmed using the JGI QC Pipeline. Plate-based RNA sample prep was performed on the PerkinElmer Sciclone NGS robotic liquid handling system using Illumina's TruSeq Stranded mRNA HT sample prep kit utilizing poly-A selection of mRNA following the protocol outlined by Illumina in their user guide (https://support.illumina.com/sequencing/sequencing_kits/truseq-stranded-mrna.html) and with the following conditions: 1 ug of total RNA per sample and eight cycles of PCR for library amplification. The prepared libraries were quantified using KAPA Biosystems' next-generation sequencing library qPCR kit and run on a Roche LightCycler 480 real-time PCR instrument. Sequencing of the flowcell was performed on the Illumina NovaSeq sequencer using NovaSeq XP V1 reagent kits, S4 flowcell, following a 2x150 indexed run recipe. Raw reads were evaluated with BBDuk

434 (https://sourceforge.net/projects/bbmap/) for artifact sequences by kmer matching (kmer=25), allowing 1

435 mismatch and detected artifacts were trimmed from the 3' end of the reads. RNA spike-in reads, PhiX reads, and reads containing any Ns were removed. Quality trimming was performed using the phred trimming method set at Q6. Following trimming, reads under the length threshold were removed (minimum length 25 bases or $1 / 3$ of the original read length - whichever is longer). Filtered reads were assembled into consensus sequences using Trinity v2.3. $2^{61}$ with the -- normalize_reads (In-silico normalization routine) and --jaccard_clip (Minimizing fusion transcripts derived from gene dense genomes) options.

443 Genome annotation. Genomes were annotated using the JGI annotation pipeline ${ }^{62}$. Functional 444 annotations were obtained from InterPro ${ }^{63}, \mathrm{PFAM}^{64}$, Gene Ontology $\left(\mathrm{GO}^{65}\right)$, Kyoto Encyclopedia of 445 Genes and Genomes $\left(\mathrm{KEGG}^{66}\right)$, Eukaryotic Orthologous Groups of Proteins $\left(\mathrm{KOG}^{67}\right)$, the Carbohydrate446 Active EnZymes database $\left(\mathrm{CAZy}^{68}\right)$, MEROPS database ${ }^{69}$, the Transporter Classification Database

$447\left(\mathrm{TCDB}^{70}\right)$, and SignalP v3.0a ${ }^{71}$. CAZymes involved in the degradation of the plant cell wall were 448 separated according to ref ${ }^{72}$. Annotation information for each isolate is available through MycoCosm ${ }^{62}$.

449 We examined repetitive elements using RepeatScout ${ }^{73}$, which identifies novel repeats in the genomes, and 450 RepeatMasker (http://repeatmasker.org), which identifies known repeats based on the Repbase library ${ }^{74}$.

451 Candidate effectors were predicted using EffectorP v2.0 $0^{75}$. Genome sequencing yielded eukaryotic 452 Benchmarking Universal Single-Copy Orthologs (BUSCO) values $\geq 95 \%$ (Supplementary Table 1). On 453 average, ca. $90 \%$ of RNAseq reads mapped to each genome (Supplementary Table 1). 
Orthogroup prediction. For comparative analyses, data for 23 additional genomes of Sordariomycetes were obtained from MycoCosm ${ }^{62}$, including outgroup taxa belonging to the Hypocreales $(n=6)^{76-81}$, Glomerellales $(n=3)^{82-84}$, Sordariales $(n=2)^{85}$, Coniochaetales $(n=1)^{86}$, Togniniales $(n=1)^{87}$, Diaporthales $(n=1)^{88}$, Magnaporthales $(n=1)^{89}$, Ophiostomatales $(n=1)^{90}$, and five other families of Xylariales $(\mathrm{n}=7)^{91-93}$. Data from an additional eight taxa in Xylariaceae sensu lato ${ }^{94}$ also were obtained from MycoCosm ${ }^{62}$ (Supplementary Fig. 1; Supplementary Table 1). Orthologous gene families (i.e., orthogroups) for all 121 genomes (ingroup and outgroup) were inferred by OrthoFinder v2.3.3 ${ }^{95}$, which was executed using DIAMOND v0.9.22 ${ }^{96}$ for the all-versus-all sequence similarity search and MAFFT $\mathrm{v} 7.427^{97}$ for sequence alignment.

Phylogenomic analysis. Protein sequences of 1,526 single-copy orthogroups defined by OrthoFinder were aligned using MAFFT v7.427 ${ }^{97}$, concatenated, and analyzed using maximum-likelihood in IQ-TREE multicore v1.6.11 ${ }^{98}$ with the Le Gascuel (LG) substitution model. Node support was calculated with 1,000 ultrafast bootstrap replicates. Additional phylogenomic analyses with different models of evolution, gene sets, and outgroup taxa resulted in nearly identical topologies (see Supplementary Materials; Supplementary Fig. 12).

Analysis and functional annotation of orthologous gene families. Representative annotations from InterPro $^{63}, \mathrm{PFAM}^{64}, \mathrm{GO}^{65}, \mathrm{CAZy}^{68}, \mathrm{MEROPS}^{69}$ and $\mathrm{TCDB}^{70}$, SignalP $3.0^{71}$, and EffectorP $2.0^{75}$ were assigned to orthogroups KinFin $v 1.0^{99}$. The criteria for orthogroup annotation was (i) a minimum of $75 \%$ of the proteins in the orthogroup share the annotation and (ii) $30 \%$ of the taxa in the cluster with at least one protein annotated with that domain. KinFin was used to aid in the classification of orthogroups into different categories such as isolate-specific, subfamily-specific, and universal (Supplementary Fig. 2e; Supplementary Table 7) and to identify orthogroups that were significantly enriched or depleted in the two major clades (i.e., Xylariaceae and Hypoxylaceae) using the Mann-Whitney U test (Supplementary Fig. 8a). We also compared functional categories for universal (i.e,. "core”) vs. isolate-specific (i.e., "dispensable") orthogroups using euKaryotic Orthologous Groups (KOGs) (Supplementary Fig. 13;

482 Supplementary Table 3f). We also used KinFin to generate a network representation of the OrthoFinder 483 clustering. The resulting network was edited with Gephi v0.9.1 ${ }^{100}$, whereby nodes were positioned by a 484 force directed layout algorithm (as described by ref ${ }^{99}$ ) (Supplementary Fig. 14). reconstruction in Count v10.04 ${ }^{101}$ with the unweighted Wagner parsimony method (gain and loss 
penalties both set to 1) to assess changes in the size of orthologous gene families over evolutionary time. This gene tree unaware method requires as input the organismal phylogeny and a gene family size table showing the numbers of genes per orthogroup per taxa, estimates the gene family sizes, as well as orthogroup gain and loss events at ancestral nodes. An orthogroup gain is defined as a shift from orthogroup absence at the preceding node to presence at the node of interest, and orthogroup loss is the opposite transition. Functional annotation of orthogroups was imported into Count v10.04 GUI to assist in the interpretation of the results. The ancestral gene content was reconstructed for the entire data set, as well as for subsets of orthologous gene families corresponding to CAZymes and PCWDEs (see Supplementary Fig. 15).

Metabolic gene cluster prediction. SMGCs were predicted using antiSMASH version $5.1 .0^{26}$ setting the strictness to 'relaxed' and enabling 'KnownClusterBlast', 'ClusterBlast', 'SubClusterBlast', 'ActiveSiteFinder', 'Cluster Pfam analysis' and 'Pfam-based GO term annotation'. Clinker and clustermap.js were used to visualize and compare $\mathrm{SMGCs}^{102}$. Sequence similarity network analysis of the SMGCs was performed using the Biosynthetic Gene Similarity Clustering and Prospecting Engine (BiGSCAPE) v1.0.1 ${ }^{103}$. BiG-SCAPE was executed under the hybrid mode, enabling the inclusion of singletons and the SMGCs from the Minimum Information about a Biosynthetic Gene cluster (MIBiG) repository version $1.4^{30}$. To compare the distribution of SMGCs, BiG-SCAPE families representing different SMGC types were combined into a single dataset. To remove duplicates, SMGCs assigned to multiple families were arbitrarily assigned to the largest family. The output from BiG-SCAPE was also incorporated into $\mathrm{KinFin}^{99}$ to visualize gene content similarity as network graphs (Fig. 2d) as well as examine SMGC distribution across clades (Fig. 2b). We observed no correlation of SMGC content and the number of scaffolds per genome (Supplementary Fig. 16).

To examine metabolic gene clusters involved in catabolism (i.e., catabolic gene clusters; CGCs)

512 we used cluster_retrieve (https://github.com/egluckthaler/cluster_retrieve) to search for clusters

513 containing phenylpropanoid degradation "anchor" genes ${ }^{27}$. Cluster_retrieve searches for multiple "cluster

514 models" containing one of 13 anchor genes: aromatic ring-opening dioxygenase (ard), benzoate 4-

515 monooxygenase (bph), ferulic acid esterase 7 (cae), catechol dioxygenase (cch), epicatechin laccase (ecl),

516 ferulic acid decarboxylase (fad), pterocarpan hydroxylase (mak), naringenin 3-dioxygenase (nad), phenol

517 2-monooxygenase (pmo), quinate 5-dehydrogenase (qdh), salicylate hydroxylase (sah), stilbene

518 dioxygenase (sdo), and vanillyl alcohol oxidase (vao) ${ }^{27}$. Homologous genes in each locus were defined by

519 a minimum BLASTp (v2.2.25+) bitscore of 50 and 30\% amino acid identity, and target sequence

520 alignment $50-150 \%$ of the query sequence length. Homologs of query genes were considered clustered if

521 separated by no more than six intervening genes. Clusters on the same contig were consolidated if 
522 separated by less than $30 \mathrm{~kb}$ and homologous cluster families across genomes were inferred using a

523 modified version of BiG-SCAPE ${ }^{103}$. We adapted the BiG-SCAPE network model for catabolic clusters by

524 adding catabolic anchor genes to "anchor_domains.txt" and manually tuning the "Others" cluster type

525 model parameters until known related clusters, such as quinate dehydrogenase clusters, merged into

526 families. Tuning resulted in the values 0.35 for the Jaccard dissimilarity of cluster Pfams, 0.63 for Pfam

527 sequence similarity, 0.02 adjacency index, and 2.0 anchor boost.

529 Detection of HGT events. We used the Alien Index (AI) pipeline

530 (https://github.itap.purdue.edu/jwisecav/wise) as previously described (see refs ${ }^{33,104}$ ) to identify HGT

531 candidates across the 121 genomes. Each predicted protein sequence was queried against a custom protein

532 database using Diamond v0.9.22.123 ${ }^{96}$. The custom database consisted of NCBI RefSeq (release 98$)^{105}$

533 supplemented with additional predicted protein sequences from the Marine Microbial Eukaryotic

534 Transcriptome Sequencing Project (MMETSP) ${ }^{106}$ and the 1000 Plants transcriptome sequencing project

$535(\mathrm{OneKP})^{107}$. Diamond results were sorted based on the normalized bitscore ( $\left.n b s\right)$, where $n b s$ was

536 calculated as the bitscore of the single best high scoring segment pair (HSP) in the hit sequence divided

537 by the best bitscore possible for the query sequence (i.e., the bitscore of the query aligned to itself).

To identify HGT candidates, an ancestral lineage is first specified, and the AI score calculated using the formula: $\mathrm{AI}=n b s O-n b s A$, where $n b s O$ is the normalized bit score of the best hit to a species outside of the ancestral lineage and $n b s A$ is the normalized bit score of the best hit to a species within the ancestral lineage. AI scores range from -1 to 1 , being greater than zero if the predicted protein sequence

542 had a better hit to species outside of the ancestral lineage and can be suggestive of either HGT or 543 contamination $^{33}$. To identify HGTs present in multiple species, a recipient sub-lineage within the larger 544 ancestral lineage may also be specified to identify their shared HGT candidates (Supplementary Fig. 3). 545 All hits to the recipient lineage are skipped so as not to be included in the $n b s A$ calculation. To identify 546 candidate HGTs acquired from distant gene donors (e.g. viruses, bacteria, or plants) we first ran the AI 547 pipeline using Ascomycota (NCBI:txid4890) and Xylariomycetidae (NCBI:txid 222545) as the ancestral 548 and recipient lineages, respectively (Supplementary Fig. 3). To identify candidate horizontal transfers of 549 genes predicted by antiSMASH to be in a SMGC from more closely related donors (e.g., other

550 filamentous fungi), we ran the AI pipeline a second time using Xylariales (NCBI:txid 37989) as the 551 ancestral lineage and manually curated subclades (see Supplementary Table 1) as recipient lineages (see 552 Supplementary Fig. 3).

553 All HGT candidates were selected for tree building if they passed the following filters: (i) AI 554 score of greater than 0 , (ii) significant hits to at least 25 sequences in the custom database, and (iii) at least $55550 \%$ of top hits were to sequences outside of the ancestral lineage. Full-length proteins corresponding to 
the top $<200$ hits $\left(\right.$ E-value $\left.<1 \times 10^{-3}\right)$ to each AI candidate were extracted from the custom database using esl-sfetch ${ }^{108}$. Sequences were aligned using MAFFT v7.407 using --auto to select the best alignment strategy ${ }^{97}$. The number of well aligned columns was determined with trimAL v.1.4. rev15 using its gappyout strategy ${ }^{109}$ only alignments with $\geq 50$ retained columns after trimAL were retained for phylogenetic analysis. Phylogenetic trees were constructed using the untrimmed MAFFT alignment as input using IQ-TREE v1.6.10 ${ }^{98}$ using the built in ModelFinder to determine the best-fit substitution model $^{110}$ and performing SH-aLRT and ultrafast bootstrapping analyses with 1,000 replicates each. Phylogenies were visualized using iTOL $v 4^{111}$.

Although the case for HGT is strongest when genes suspected of being horizontally acquired have well-supported phylogenetic associations that contradict accepted species relationships ${ }^{112}$, our initial query-based trees often lacked sufficient taxon sampling to be incongruent with the accepted species phylogeny. This was frequently the case when evaluating candidate HGTs from distant donors. For example, a query-based gene tree may contain only the recipient Xylariales and bacterial sequences but lacked sequences from other filamentous fungi. Sequences from other fungi add important context to indicate where the Xylariales sequences would have grouped if inherited vertically. Therefore, for identifying high confidence transfers from distant donors (i.e., first AI analysis), we combined the AI and

572 OrthoFinder analyses to construct trees containing homologous sequences from additional fungi. For

573 orthogroups with one or more AI candidates, we combined all orthogroup sequences with all extracted top

574 hits to each AI candidate. Sequences were aligned and trees constructed as described above. Each

575 phylogenetic tree was then manually curated to verify HGT with either high or low confidence. To be

576 considered a high confidence candidate, HGT events had to meet the following criteria: (i) the association

577 between donor and recipient clades was supported by ultrafast bootstrap $>=95$ and (ii) recipient clade

578 consisted of sequences from two or more species. If the candidate met one of the two criteria, the HGT

579 was considered low confidence. Phylogenies that that not meet these criteria were excluded.

581 Statistical analyses. To assess whether genes within different functional categories are associated with 582 ecological mode (endophytic and non-endophytic), we performed phylogenetically independent contrasts

583 (PICs ${ }^{113}$ ) with the function 'brunch' of the package 'caper' version 1.0.1 ${ }^{114}$ in R version 3.6.1

584 (Supplementary Table 6). All other statistics were done in R version 3.6.1 or JMP version 15.1 (SAS

585 Institute Inc., Cary, NC).

Data availability. Raw sequence data, assembled sequences, and genome annotations are available 
for raw reads and assemblies are listed in Supplementary Table 1. All other data can be found in FigShare Repository (DOI 10.6084/m9.figshare.c.5314025).

\section{REFERENCES}

1. Peay, K. G., Kennedy, P. G. \& Talbot, J. M. Dimensions of biodiversity in the Earth mycobiome. Nat. Rev. Microbiol. 14, 434-447 (2016).

2. U'Ren, J. M., Lutzoni, F., Miadlikowska, J., Laetsch, A. D. \& Arnold, A. E. Host and geographic structure of endophytic and endolichenic fungi at a continental scale. Am. J. Bot. 99, 898-914 (2012).

3. U'Ren, J. M. et al. Host availability drives distributions of fungal endophytes in the imperiled boreal realm. Nature Ecology and Evolution 3, 1430-1437 (2019).

4. Rodriguez, R. J., White, J. F., Jr, Arnold, A. E. \& Redman, R. S. Fungal endophytes: diversity and functional roles: Tansley review. New Phytol. 182, 314-330 (2009).

5. Arnold, A. E. et al. A phylogenetic estimation of trophic transition networks for ascomycetous fungi:

6. Porras-Alfaro, A. \& Bayman, P. Hidden fungi, emergent properties: endophytes and microbiomes. Annu. Rev. Phytopathol. 49, 291-315 (2011).

7. U'Ren, J. M. et al. Contributions of North American endophytes to the phylogeny, ecology, and taxonomy of Xylariaceae (Sordariomycetes, Ascomycota). Mol. Phylogenet. Evol. 98, 210-232 (2016).

8. Stadler, M., Kuhnert, E., Peršoh, D. \& Fournier, J. The Xylariaceae as model example for a unified nomenclature following the 'One Fungus-One Name' (1F1N) concept. Mycology 3, 5-21 (2013). rDNA sequence analyses with saprobes forming fruit bodies in the field. Mycoscience 49, 359-372 (2008). 
10. Becker, K. \& Stadler, M. Recent progress in biodiversity research on the Xylariales and their secondary metabolism. J. Antibiot. 74, 1-23 (2021).

618 11. Rokas, A., Wisecaver, J. H. \& Lind, A. L. The birth, evolution and death of metabolic gene clusters 619 in fungi. Nat. Rev. Microbiol. 16, 731-744 (2018).

620 12. Wibberg, D. et al. High quality genome sequences of thirteen Hypoxylaceae (Ascomycota)

621 strengthen the phylogenetic family backbone and enable the discovery of new taxa. Fungal Divers.

$622 \quad(2020)$.

623 13. Nielsen, J. C. et al. Global analysis of biosynthetic gene clusters reveals vast potential of secondary 624 metabolite production in Penicillium species. Nat Microbiol 2, 17044 (2017).

625 14. Drott, M. T. et al. Microevolution in the pansecondary metabolome of Aspergillus flavus and its potential macroevolutionary implications for filamentous fungi. Proc. Natl. Acad. Sci. U. S. A. 118, (2021).

15. Scharf, D. H., Heinekamp, T. \& Brakhage, A. A. Human and plant fungal pathogens: the role of secondary metabolites. PLoS Pathog. 10, e1003859 (2014).

16. Sumarah, M. W. \& Miller, J. D. Anti-insect secondary metabolites from fungal endophytes of conifer trees. Nat. Prod. Commun. 4, 1497-1504 (2009).

17. Slot, J. C. Fungal Gene Cluster Diversity and Evolution. Adv. Genet. 100, 141-178 (2017).

18. Pusztahelyi, T., Holb, I. J. \& Pócsi, I. Secondary metabolites in fungus-plant interactions. Front. Plant Sci. 6, 573 (2015).

19. Wisecaver, J. H. \& Rokas, A. Fungal metabolic gene clusters - caravans traveling across genomes and environments. Front. Microbiol. 6, 161 (2015).

638 plant pathogenic fungi. BMC Biol. 14, 41 (2016).

639 21. Ju, Y. M. \& Rogers, J. D. A revision of the genus Hypoxylon. Mycologia Memoir no. 20. St. Paul

$640 \quad(M N):$ APS Press (1996).

641 22. Voglmayr, H., Friebes, G., Gardiennet, A. \& Jaklitsch, W. M. Barrmaelia and Entosordaria in 
Barrmaeliaceae (fam. nov., Xylariales) and critical notes on Anthostomella -like genera based on multigene phylogenies. Mycol. Prog. 17, 155-177 (2018).

644 23. Wendt, L. et al. Resurrection and emendation of the Hypoxylaceae, recognised from a multigene 645 phylogeny of the Xylariales. Mycol. Prog. 17, 115-154 (2018).

646 24. Shen, X.-X. et al. Genome-scale phylogeny and contrasting modes of genome evolution in the 647 fungal phylum Ascomycota. Sci Adv 6, (2020).

648 25. Miyauchi, S. et al. Large-scale genome sequencing of mycorrhizal fungi provides insights into the 649 early evolution of symbiotic traits. Nat. Commun. 11, 5125 (2020).

650 26. Blin, K. et al. antiSMASH 5.0: updates to the secondary metabolite genome mining pipeline. Nucleic Acids Res. 47, W81-W87 (2019).

27. Gluck-Thaler, E., Vijayakumar, V. \& Slot, J. C. Fungal adaptation to plant defences through convergent assembly of metabolic modules. Mol. Ecol. 27, 5120-5136 (2018).

28. Gluck-Thaler, E. et al. The architecture of metabolism maximizes biosynthetic diversity in the largest class of fungi. Mol. Biol. Evol. 37, 2838-2856 (2020).

29. Gluck-Thaler, E. \& Slot, J. C. Specialized plant biochemistry drives gene clustering in fungi. ISME J. 12, 1694-1705 (2018).

30. Medema, M. H. et al. Minimum information about a biosynthetic gene cluster. Nat. Chem. Biol. 11, 625-631 (2015).

31. Ambrose, K. V. et al. Functional characterization of salicylate hydroxylase from the fungal endophyte Epichloë festucae. Sci. Rep. 5, 10939 (2015).

32. Alexander, W. G., Wisecaver, J. H., Rokas, A. \& Hittinger, C. T. Horizontally acquired genes in early-diverging pathogenic fungi enable the use of host nucleosides and nucleotides. Proc. Natl. Acad. Sci. U. S. A. 113, 4116-4121 (2016).

33. Wisecaver, J. H., Alexander, W. G., King, S. B., Hittinger, C. T. \& Rokas, A. Dynamic evolution of nitric oxide detoxifying flavohemoglobins, a family of single-protein metabolic modules in bacteria and eukaryotes. Mol. Biol. Evol. 33, 1979-1987 (2016). 
34. Gonçalves, C. et al. Evidence for loss and reacquisition of alcoholic fermentation in a fructophilic yeast lineage. Elife 7, (2018).

35. Lawrence, D. P., Kroken, S., Pryor, B. M. \& Arnold, A. E. Interkingdom gene transfer of a hybrid NPS/PKS from bacteria to filamentous Ascomycota. PLoS One 6, e28231 (2011).

36. Marcet-Houben, M. \& Gabaldón, T. Acquisition of prokaryotic genes by fungal genomes. Trends Genet. 26, 5-8 (2010).

37. Massengo-Tiassé, R. P. \& Cronan, J. E. Diversity in enoyl-acyl carrier protein reductases. Cell. Mol. Life Sci. 66, 1507-1517 (2009).

38. Blatzer, M. et al. SidL, an Aspergillus fumigatus transacetylase involved in biosynthesis of the siderophores ferricrocin and hydroxyferricrocin. Appl. Environ. Microbiol. 77, 4959-4966 (2011).

39. Medema, M. H. et al. antiSMASH: rapid identification, annotation and analysis of secondary metabolite biosynthesis gene clusters in bacterial and fungal genome sequences. Nucleic Acids Res. 39, W339-46 (2011).

40. Chooi, Y.-H., Cacho, R. \& Tang, Y. Identification of the viridicatumtoxin and griseofulvin gene clusters from Penicillium aethiopicum. Chem. Biol. 17, 483-494 (2010).

41. Wisecaver, J. H., Slot, J. C. \& Rokas, A. The evolution of fungal metabolic pathways. PLoS Genet. 10, e1004816 (2014).

42. Hsieh, H.-M., Ju, Y.-M. \& Rogers, J. D. Molecular phylogeny of Hypoxylon and closely related genera. Mycologia 97, 844-865 (2005).

43. Hsieh, H.-M. et al. Phylogenetic status of Xylaria subgenus Pseudoxylaria among taxa of the subfamily Xylarioideae (Xylariaceae) and phylogeny of the taxa involved in the subfamily. Molecular Phylogenetics and Evolution vol. $54957-969$ (2010).

44. Haridas, S. et al. 101 Dothideomycetes genomes: A test case for predicting lifestyles and emergence of pathogens. Stud. Mycol. 96, 141-153 (2020).

692 45. Knapp, D. G. et al. Comparative genomics provides insights into the lifestyle and reveals functional heterogeneity of dark septate endophytic fungi. Sci. Rep. 8, 6321 (2018). 
694 46. Osono, T. Role of phyllosphere fungi of forest trees in the development of decomposer fungal

695 communities and decomposition processes of leaf litter. Can. J. Microbiol. 52, 701-716 (2006).

696 47. Barberán, A. et al. Why are some microbes more ubiquitous than others? Predicting the habitat

697 breadth of soil bacteria. Ecol. Lett. 17, 794-802 (2014).

698 48. Persoh, D. et al. Molecular and morphological evidence for the delimitation of Xylaria hypoxylon.

699 Mycologia 101, 256-268 (2009).

700 49. Richards, T. A. \& Talbot, N. J. Horizontal gene transfer in osmotrophs: playing with public goods.

$701 \quad$ Nat. Rev. Microbiol. 11, 720-727 (2013).

702 50. Naranjo-Ortiz, M. A. \& Gabaldón, T. Fungal evolution: cellular, genomic and metabolic complexity.

703 Biol. Rev. Camb. Philos. Soc. (2020).

704 51. Joy, J. B. Symbiosis catalyses niche expansion and diversification. Proc. Biol. Sci. 280, 20122820

$705 \quad$ (2013).

52. Robey, M. T., Caesar, L. K., Drott, M. T., Keller, N. P. \& Kelleher, N. L. An interpreted atlas of biosynthetic gene clusters from 1,000 fungal genomes. Proc. Natl. Acad. Sci. U. S. A. 118, (2021).

53. U'Ren, J. M. \& Arnold, A. E. Diversity, taxonomic composition, and functional aspects of fungal communities in living, senesced, and fallen leaves at five sites across North America. PeerJ 4, e2768 (2016).

713 doi:10.17504/protocols.io.ga4bsgw.

714 55. Carbone, I. et al. T-BAS version 2.1: Tree-Based Alignment Selector toolkit for evolutionary 715 placement of DNA sequences and viewing alignments and specimen metadata on curated and 716 custom trees. Microbiology resource announcements 8, e00328-19 (2019).

717 56. U'Ren, J. M. \& Moore, L. P. Large volume fungal genomic DNA extraction protocol for PacBio. $718 \quad$ Protocols.io dx.doi.org/10.17504/protocols.io.qtjdwkn

719 57. U’Ren, J. M. \& Moore, L. Small volume fungal genomic DNA extraction protocol for Illumina 
genome. Protocols.io dx.doi.org/10.17504/protocols.io.3w9gph6

721 58. Chu, G., Vollrath, D. \& Davis, R. W. Separation of large DNA molecules by contour-clamped

722 homogeneous electric fields. Science 234, 1582-1585 (1986).

723 59. Luo, M. \& Wing, R. A. An improved method for plant BAC library construction. Methods Mol. Biol.

$724 \quad$ 236, 3-20 (2003).

60. Bankevich, A. et al. SPAdes: a new genome assembly algorithm and its applications to single-cell sequencing. J. Comput. Biol. 19, 455-477 (2012).

61. Grabherr, M. G. et al. Full-length transcriptome assembly from RNA-Seq data without a reference genome. Nat. Biotechnol. 29, 644-652 (2011).

62. Grigoriev, I. V. et al. MycoCosm portal: gearing up for 1000 fungal genomes. Nucleic Acids Res. 42, D699-704 (2014).

63. Mitchell, A. L. et al. InterPro in 2019: improving coverage, classification and access to protein sequence annotations. Nucleic Acids Res. 47, D351-D360 (2019).

64. El-Gebali, S. et al. The Pfam protein families database in 2019. Nucleic Acids Res. 47, D427-D432 (2019).

65. The Gene Ontology Consortium. The Gene Ontology Resource: 20 years and still GOing strong. Nucleic Acids Res. 47, D330-D338 (2019).

66. Kanehisa, M. et al. From genomics to chemical genomics: new developments in KEGG. Nucleic Acids Res. 34, D354-7 (2006).

67. Tatusov, R. L. et al. The COG database: an updated version includes eukaryotes. $B M C$ Bioinformatics 4, 41 (2003).

68. Lombard, V., Golaconda Ramulu, H., Drula, E., Coutinho, P. M. \& Henrissat, B. The carbohydrateactive enzymes database (CAZy) in 2013. Nucleic Acids Res. 42, D490-5 (2014).

743 69. Rawlings, N. D., Barrett, A. J. \& Finn, R. Twenty years of the MEROPS database of proteolytic enzymes, their substrates and inhibitors. Nucleic Acids Res. 44, D343-50 (2016).

745 70. Saier, M. H., Jr et al. The Transporter Classification Database (TCDB): recent advances. Nucleic 
Acids Res. 44, D372-9 (2016).

747 71. Nielsen, H. Predicting Secretory Proteins with SignalP. Methods Mol. Biol. 1611, 59-73 (2017).

748 72. Kameshwar, A. K. S., Ramos, L. P. \& Qin, W. CAZymes-based ranking of fungi (CBRF): an interactive web database for identifying fungi with extrinsic plant biomass degrading abilities. Bioresources and Bioprocessing 6, 51 (2019).

73. Price, A. L., Jones, N. C. \& Pevzner, P. A. De novo identification of repeat families in large genomes. Bioinformatics 21 Suppl 1, i351-8 (2005).

74. Bao, W., Kojima, K. K. \& Kohany, O. Repbase Update, a database of repetitive elements in eukaryotic genomes. Mob. DNA 6, 11 (2015).

75. Sperschneider, J., Dodds, P. N., Gardiner, D. M., Singh, K. B. \& Taylor, J. M. Improved prediction of fungal effector proteins from secretomes with EffectorP 2.0. Mol. Plant Pathol. 19, 2094-2110 (2018).

76. Cuomo, C. A. et al. The Fusarium graminearum genome reveals a link between localized polymorphism and pathogen specialization. Science 317, 1400-1402 (2007).

77. Xiao, G. et al. Genomic perspectives on the evolution of fungal entomopathogenicity in Beauveria bassiana. Scientific Reports vol. 2 (2012).

78. Terfehr, D. et al. Genome sequence and annotation of Acremonium chrysogenum, producer of the $\beta$ Lactam antibiotic Cephalosporin C. Genome Announc. 2, (2014).

79. Wang, G. et al. Biosynthesis of antibiotic leucinostatins in Bio-control Fungus Purpureocillium lilacinum and their inhibition on phytophthora revealed by genome mining. PLoS Pathog. 12, e1005685 (2016)

80. Jourdier, E. et al. Proximity ligation scaffolding and comparison of two Trichoderma reesei strains genomes. Biotechnol. Biofuels 10, 151 (2017).

81. Druzhinina, I. S. et al. Massive lateral transfer of genes encoding plant cell wall-degrading enzymes to the mycoparasitic fungus Trichoderma from its plant-associated hosts. PLoS Genet. 14, e1007322 (2018). 
772 82. Hacquard, S. et al. Survival trade-offs in plant roots during colonization by closely related beneficial 773 and pathogenic fungi. Nat. Commun. 7, 11362 (2016).

774 83. Zampounis, A. et al. Genome sequence and annotation of Colletotrichum higginsianum, a causal 775 agent of crucifer anthracnose Disease. Genome Announc. 4, (2016).

776 84. Grum-Grzhimaylo, A. A. et al. The obligate alkalophilic soda-lake fungus Sodiomyces alkalinus has 777 shifted to a protein diet. Mol. Ecol. 27, 4808-4819 (2018).

778 85. Berka, R. M. et al. Comparative genomic analysis of the thermophilic biomass-degrading fungi Myceliophthora thermophila and Thielavia terrestris. Nat. Biotechnol. 29, 922-927 (2011).

86. Jiménez, D. J. et al. Draft genome sequence of Coniochaeta ligniaria NRRL 30616, a lignocellulolytic fungus for bioabatement of inhibitors in plant biomass hydrolysates. Genome Announc. 5, (2017).

87. Blanco-Ulate, B., Rolshausen, P. \& Cantu, D. Draft genome sequence of the ascomycete Phaeoacremonium aleophilum strain UCR-PA7, a causal agent of the Esca disease complex in grapevines. Genome Announc. 1, (2013).

88. Morales-Cruz, A. et al. Distinctive expansion of gene families associated with plant cell wall degradation, secondary metabolism, and nutrient uptake in the genomes of grapevine trunk pathogens. BMC Genomics 16, 469 (2015).

89. Dean, R. A. et al. The genome sequence of the rice blast fungus Magnaporthe grisea. Nature 434, 980-986 (2005).

90. Forgetta, V. et al. Sequencing of the Dutch elm disease fungus genome using the Roche/454 GS-

91. Blanco-Ulate, B., Rolshausen, P. E. \& Cantu, D. Draft genome sequence of the grapevine dieback FLX Titanium System in a comparison of multiple genomics core facilities. J. Biomol. Tech. 24, 3949 (2013). fungus Eutypa lata UCR-EL1. Genome Announcements vol. 1 (2013).

92. David, A. S. et al. Draft genome sequence of Microdochium bolleyi, a dark septate fungal endophyte of beach grass. Genome Announc. 4, (2016). 
93. Mondo, S. J. et al. Widespread adenine N6-methylation of active genes in fungi. Nat. Genet. 49, 964-968 (2017).

94. Wu, W. et al. Characterization of four endophytic fungi as potential consolidated bioprocessing hosts for conversion of lignocellulose into advanced biofuels. Appl. Microbiol. Biotechnol. 101, 26032618 (2017).

95. Emms, D. M. \& Kelly, S. OrthoFinder: phylogenetic orthology inference for comparative genomics. Genome Biol. 20, 238 (2019).

96. Buchfink, B., Xie, C. \& Huson, D. H. Fast and sensitive protein alignment using DIAMOND. Nat. Methods 12, 59-60 (2015).

97. Katoh, K. \& Standley, D. M. MAFFT multiple sequence alignment software version 7: improvements in performance and usability. Mol. Biol. Evol. 30, 772-780 (2013).

98. Nguyen, L.-T., Schmidt, H. A., von Haeseler, A. \& Minh, B. Q. IQ-TREE: a fast and effective stochastic algorithm for estimating maximum-likelihood phylogenies. Mol. Biol. Evol. 32, 268-274

99. Laetsch, D. R. \& Blaxter, M. L. KinFin: Software for taxon-aware analysis of clustered protein sequences. G3 7, 3349-3357 (2017).

814 100. Bastian, M., Heymann, S. \& Jacomy, M. Gephi: an open source software for exploring and manipulating networks. in Third international AAAI conference on weblogs and social media

817 101. Csurös, M. Count: evolutionary analysis of phylogenetic profiles with parsimony and likelihood.

818 Bioinformatics 26, 1910-1912 (2010).

819 102. Gilchrist, C. L. M. \& Chooi, Y.-H. clinker \& clustermap.js: Automatic generation of gene cluster $820 \quad$ comparison figures. Cold Spring Harbor Laboratory 2020.11.08.370650 (2020)

821 doi:10.1101/2020.11.08.370650.

822 103. Navarro-Muñoz, J. C. et al. A computational framework to explore large-scale biosynthetic 823 diversity. Nat. Chem. Biol. 16, 60-68 (2020). 
824 104. Verster, K. I. et al. Horizontal transfer of bacterial cytolethal distending toxin B genes to insects. Mol. Biol. Evol. 36, 2105-2110 (2019).

105. O’Leary, N. A. et al. Reference sequence (RefSeq) database at NCBI: current status, taxonomic expansion, and functional annotation. Nucleic Acids Res. 44, D733-45 (2016).

106. Keeling, P. J. et al. The Marine Microbial Eukaryote Transcriptome Sequencing Project (MMETSP): illuminating the functional diversity of eukaryotic life in the oceans through transcriptome sequencing. PLoS Biol. 12, e1001889 (2014).

107. Matasci, N. et al. Data access for the 1,000 Plants (1KP) project. Gigascience 3, 17 (2014).

108. Eddy, S. R. A new generation of homology search tools based on probabilistic inference. Genome

$$
\text { Inform. 23, 205-211 (2009). }
$$

109. Capella-Gutiérrez, S., Silla-Martínez, J. M. \& Gabaldón, T. trimAl: a tool for automated alignment trimming in large-scale phylogenetic analyses. Bioinformatics 25, 1972-1973 (2009). fast model selection for accurate phylogenetic estimates. Nat. Methods 14, 587-589 (2017). Nucleic Acids Res. 47, W256-W259 (2019).

112. Soanes, D. \& Richards, T. A. Horizontal gene transfer in eukaryotic plant pathogens. Annu. Rev. Phytopathol. 52, 583-614 (2014).

113. Felsenstein, J. Phylogenies and the comparative Method. Am. Nat. 125, 1-15 (1985).

114. Orme, D. et al. Package 'caper'. Reference manual, available at 467, (2012).

846 Funding for the project was provided by the DOE JGI Large-scale Community Science Project (Grant 847 number 503506). MEEF was funded by the Office for Research, Innovation and Impact at the University 848 of Arizona and the University of Arizona BIO5 Postdoctoral Fellowship Program. FL and JM

849 acknowledge the financial support from the NSF DEB-1541548 and DEB-1046065. We thank F. Martin, 850 P. Gladieux, J. Spatafora, R. Vilgalys, and K. O`Donnell for permission to use unpublished JGI F1000 
851 genomes; D. Bellomo, Y. Sanchez-Rosario, and S. Valdez for laboratory assistance; and the Genomics

852 Analysis and Sequencing Core (GATC), the Arizona Genomics Institute (AGI), and the High-

853 Performance Computer (HPC) at the University of Arizona for technical support.

854

\section{AUTHOR CONTRIBUTIONS}

856 Designed research: JMU, JHW, AEA, MEEF; Performed field or laboratory research: JMU, LPM, YMJ, 857 AEA, FL, JM; Contributed fungal isolates or analytic tools: YMJ, DCE, RM, JHW, JCS, KYC, JGI

858 authors; Analyzed data: MEEF, JMU, JHW, KEE, KS, ZK, JGI authors; Wrote the paper: MEEF, JMU, 859 JHW, with contributions from all authors.

860

\section{COMPETING INTERESTS}

862 The authors declare no competing interests. 
a

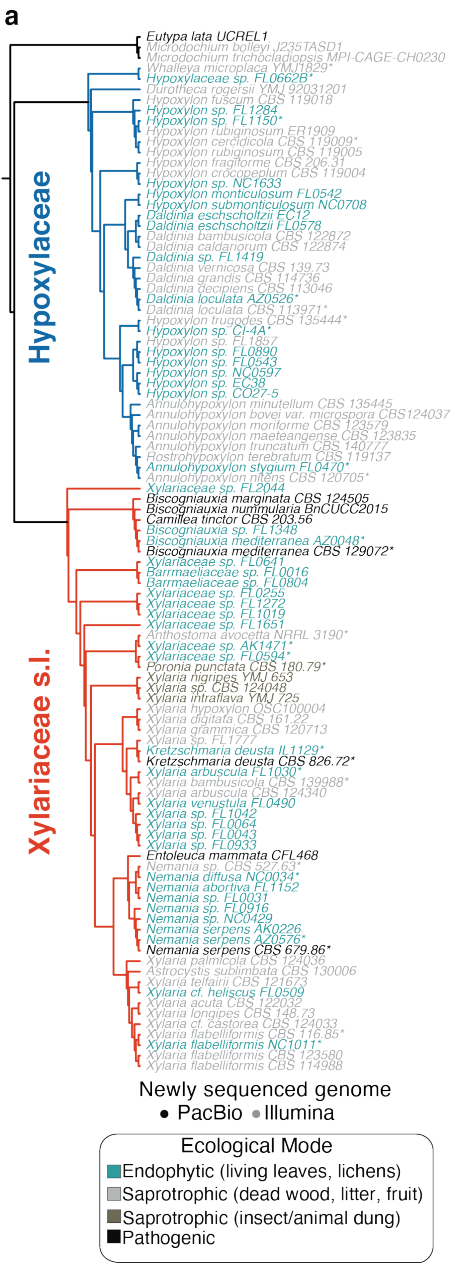

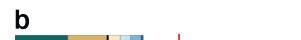

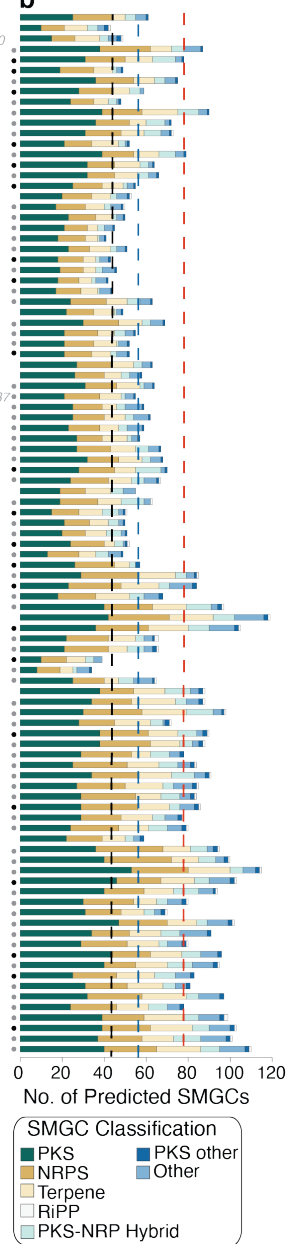

c

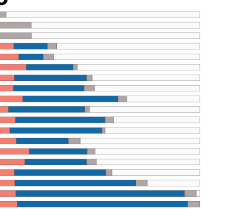

d
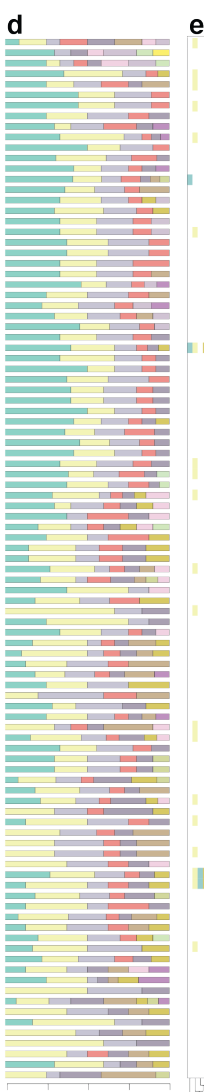

In

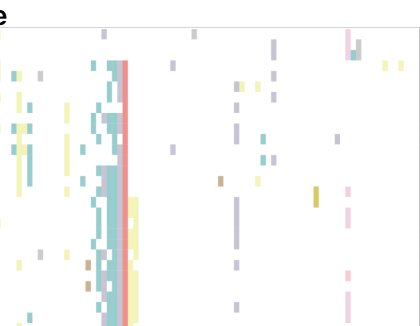

$20 \quad 40 \quad 60 \quad 80 \quad 100$

Relative Abundance (\%) SMGC Distribution
Specific to: Specific to: - Xylariaceae/Hypoxylaceae Xypoxylaceae

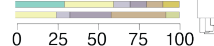

Relative Abundance (\%)

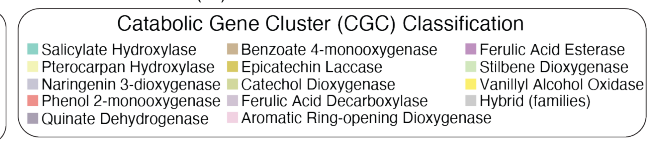

Figure 1. Xylariaceae s.l. and Hypoxylaceae genomes are characterized by hyperdiverse and dynamic metabolic gene clusters. (a) Maximum likelihood phylogenetic analyses of 1,526 universal, single-copy orthogroups support the sister relationship of the Xylariaceae s.1. ${ }^{22}$ (containing Xylariaceae sensu stricto and Graphostromataceae) and the Hypoxylaceae ${ }^{23}$ (Fig. 1a; Supplementary Figs. 1,2), as well as previously denoted relationships among genera ${ }^{7}$. Phylogenetic analyses included genomes of 25 outgroup taxa representing five other families of Xylariales and eight orders of Sordariomycetes (total 121 genomes; Supplementary Fig. 1). Taxon names are colored by ecological mode and branches colored by major clade (red: Xylariaceae s.l.; blue: Hypoxylaceae). Taxa with asterisks $\left({ }^{*}\right)$ represent 15 pairs of endophyte/non-endophyte sister taxa used to assess differences in genomic content due to ecological mode (see Fig. 5). Within this phylogenetic framework, we compared the: (b) abundance of different SMGC families per genome. Dotted lines indicate the averages for Pezizomycotina (black), Xylariaceae s.l. (red), and Hypoxylaceae (blue); (c) relative abundance of family-specific, clade-specific, and isolatespecific SMGCs; (d) relative abundance and (e) presence/absence of catabolic gene clusters (CGCs), colored by anchor gene identity $\left(\mathrm{sensu}^{29}\right)$. Hierarchical clustering of CGCs (see bottom) was performed 881 with the unweighted pair group method with arithmetic mean (UPGMA). 
bioRxiv preprint doi: https://doi.org/10.1101/2021.06.01.446356; this version posted June 2, 2021. The copyright holder for this preprint (which was not certified by peer review) is the author/funder, who has granted bioRxiv a license to display the preprint in perpetuity. It is made available under aCC-BY-NC-ND 4.0 International license.
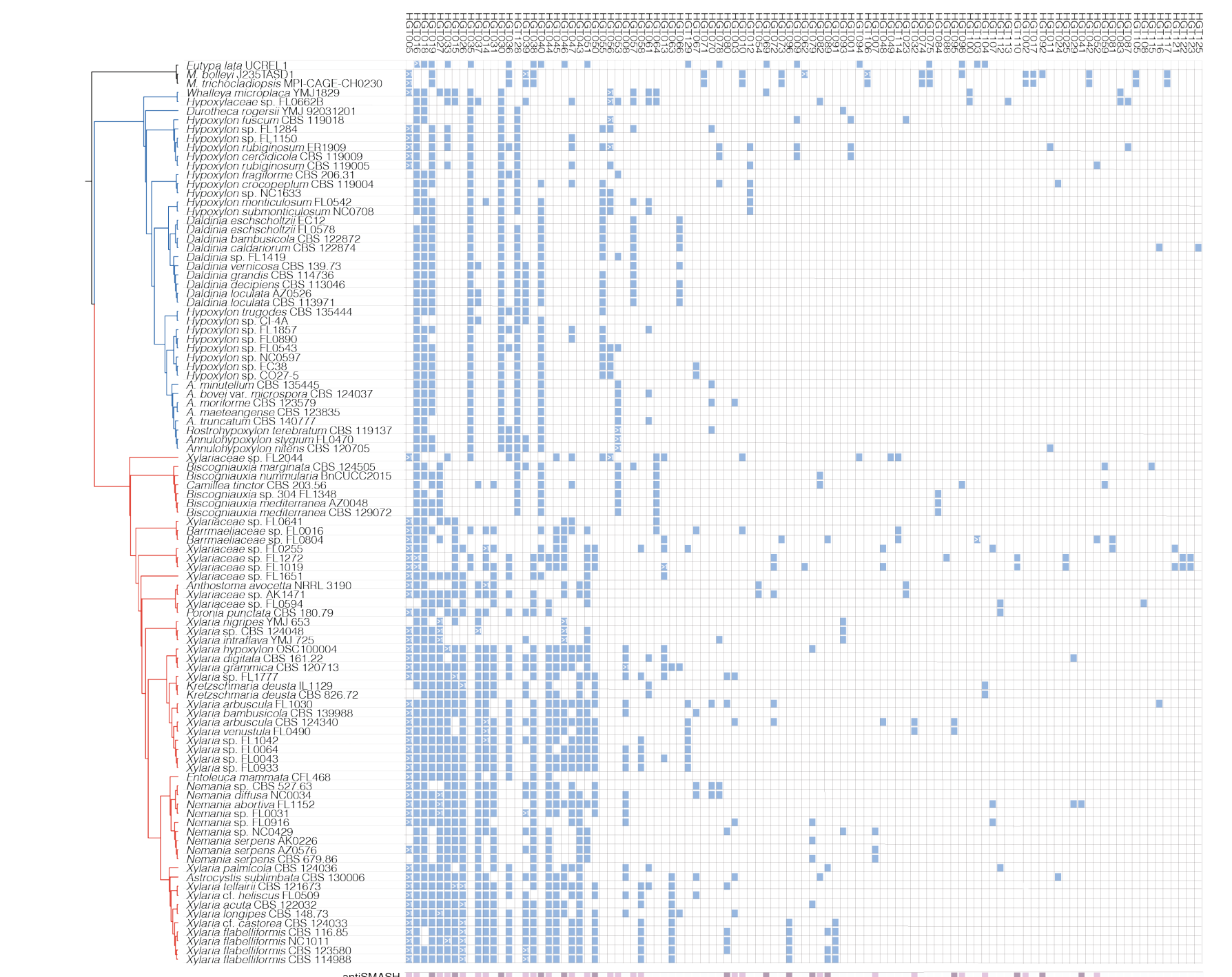

Functional annotation

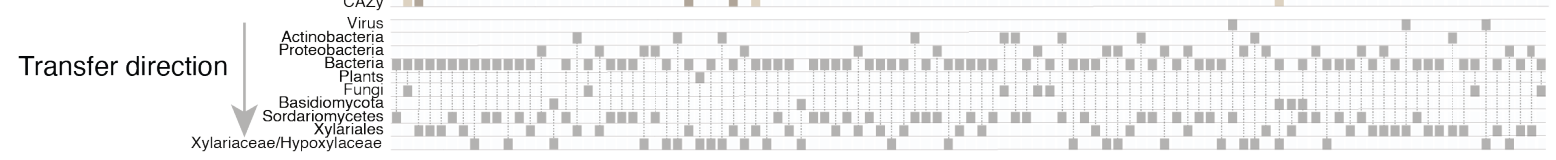
of Xylariaceae and Hypoxylaceae. Phylogeny matches Fig. 1a. Blue boxes represent genes predicted to be high-confidence HGT events (detected with the first round of Alien Index analyses; Supplementary Table 5). HGT events are ordered from left to right based on their abundance. Transfers with more than one gene copy per genome are indicated with $>1$. Functional annotations (bottom) are based on antiSMASH, EffectorP, SignalP, TCDB, MEROPS, and CAZyme. SMGCs predicted as 'biosyntheticcore' and 'biosynthetic-additional' are shown with darker purple, whereas other genes in SMGCs are shown with light purple. For CAZyme predictions, dark brown color indicates plant cell wall-degrading carbohydrate-active enzyme domains (PCWDs). 

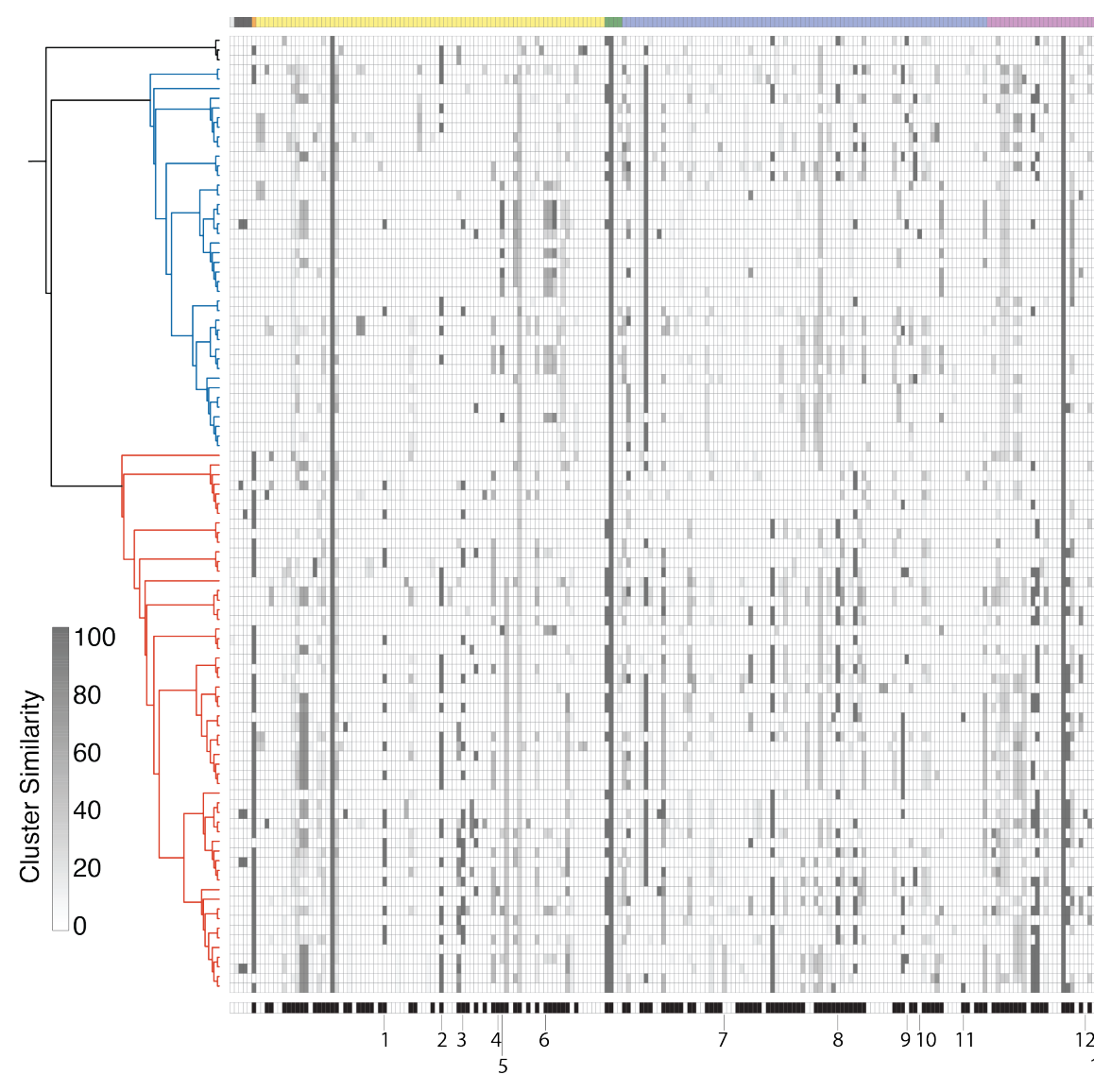

MIBiG Taxonomy

\section{NA}

Bacteria

Agaricomycetes

Sordariomycetes

Leotiomycetes

Eurotiomycetes

Dothideomycetes

Figure 3. Dynamic distribution of 168 Xylariaceae and Hypoxylaceae SMGCs with hits to known metabolites in the MIBiG repository. Rows are sorted by the taxonomic identity (class and species) of the best MIBiG hit (top). Shading indicates the similarity of predicted SMGCs to reference metabolites, defined as the percentage of genes in an SMGC with significant BLAST hits to a known SMGC in the MIBiG database ${ }^{39}$. Black boxes (bottom) indicate SMGCs predicted by Alien Index ${ }^{33,104}$ to contain at least one gene putatively transferred via HGT (Supplementary Table 5). For MIBiG clusters that occurred more than once per genome, only the hit with the highest similarity is shown (Supplementary Table 3b). 
914

915

916

917

918

919

920

921

922

923

924

925

926

927

928

929

930

931

932

933

934 b

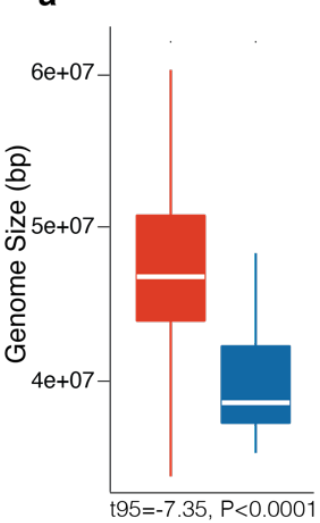

f

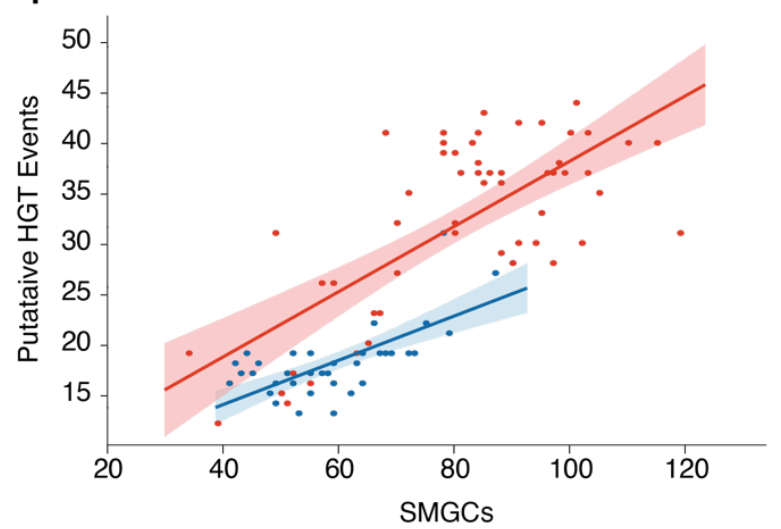

c
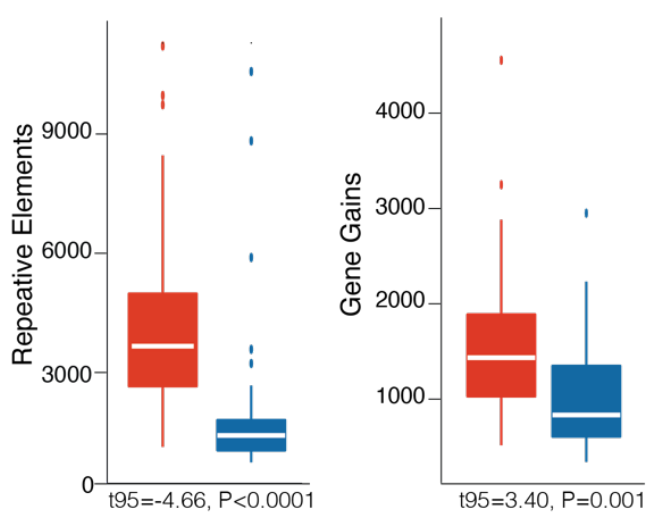

d
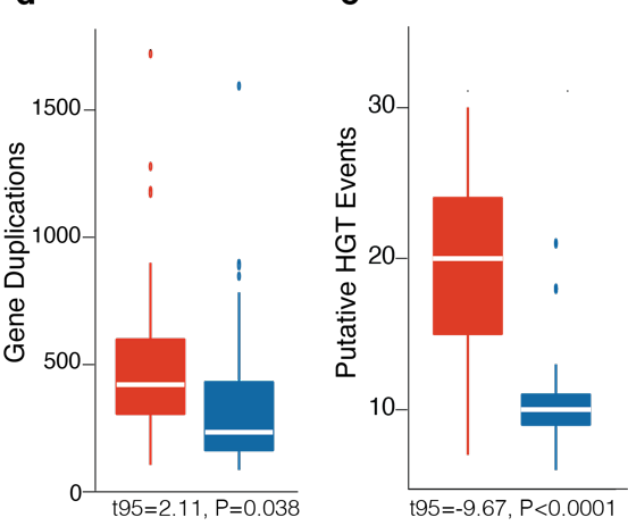

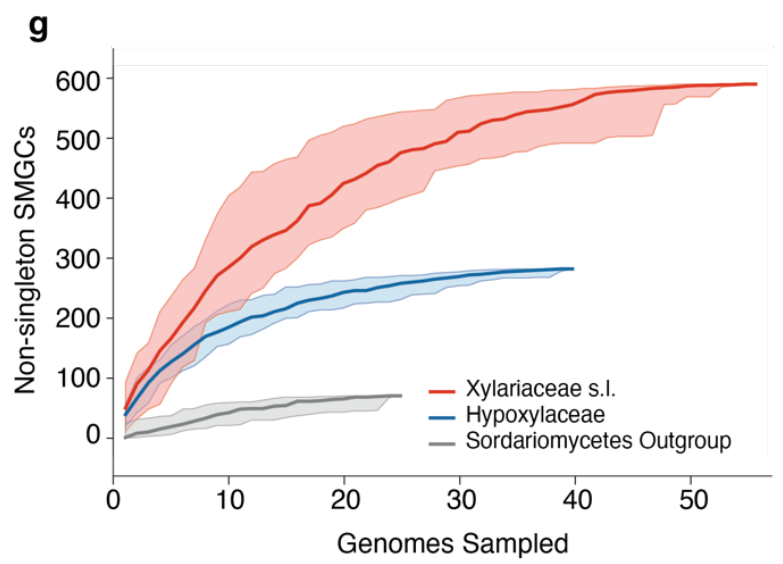

Figure 4. Larger genomes in the Xylariaceae clade reflect increased repetitive regions, gene gains and duplications, and HGTs. Median (a) genome size, (b) repetitive element content, (c) gene gains, (d) gene duplications, and (e) number of putative HGT events (high confidence only) for genomes of Xylariaceae (red) and Hypoxylaceae (blue). Box plot boundaries reflect the interquartile range. Summary statistics (averages, standard deviations, and sample sizes) are reported in Supplementary Table 6. Gene gains/losses were inferred with Wagner Parsimony under a gain penalty $=$ loss penalty $=1$; (f) Relationship between the number of HGT events and SMGCs as a function of clade (Pearson correlation for each clade was the same; $r=0.72, \mathrm{P}<0.0001$ ); (g) Rarefaction curves of non-singleton SMGCs by clade illustrates that when compared at the same number of genomes $(n=25)$, the richness of SMGCs is highest in the Xylariaceae. SMGC richness for Xylariaceae and Hypoxylaceae genomes is ca. 4-7X greater than outgroup genomes $(\mathrm{n}=71$ SMGCs). 


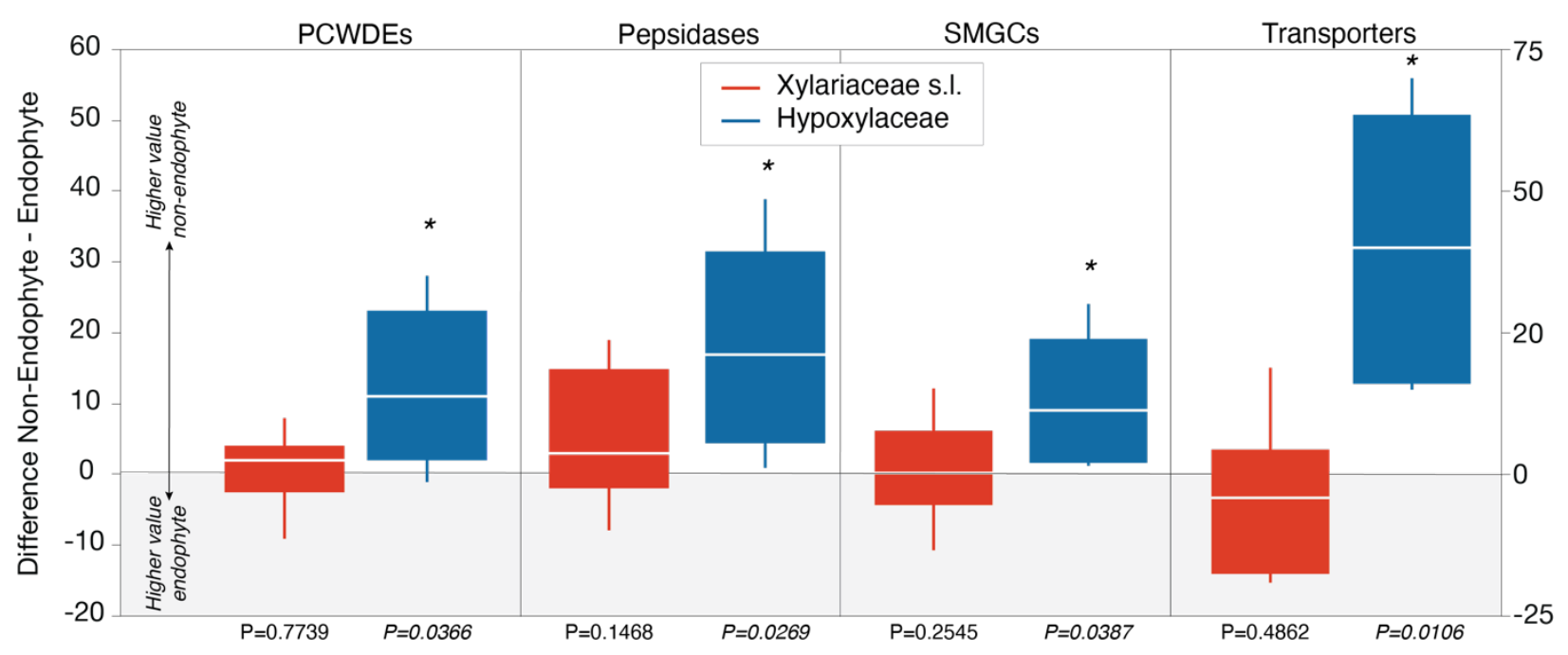

Figure 5. Pairwise comparisons of sister taxa illustrate ecological modes are more distinct in the Hypoxylaceae. Box plots of the median and interquartile difference in gene counts of PCWDEs, peptidases, SMGCs (y-axis on left), and transporters (y-axis on right) between 15 pairs of sister taxa with contrasting ecological modes for Xylariaceae and Hypoxylaceae (sister taxa are indicated with asterisks in Fig. 1a). Values greater than zero indicate higher gene counts in non-endophytic taxa, whereas differences less than zero indicate higher gene counts in endophytes. Statistical differences were assessed with least squares means contrast under the null hypothesis: non-endophyte value - endophyte value $=0$ (see Supplementary Table 6 for summary statistics). P-values $<0.05$ are indicated with an asterisk $(*)$. 


\section{SUPPLEMENTARY MATERIALS}

Functional annotation of orthogroups. All 1,451,488 genes from the 121 genomes (ingroup and outgroup) were clustered into 104,604 orthologous groups (i.e., orthogroups), and ca. $25 \%(26,825)$ were assigned functional annotations (Supplementary Table 7). Gene ontology (GO) terms were designated for 6,458 orthogroups $(6.2 \%)$, while $10,820(10.3 \%)$ and $11,144(10.7 \%)$ of gene families were assigned to InterPro and Pfam domains, respectively. A small fraction of orthogroups were assigned IDs as carbohydrate-active enzymes (CAZyme; 720, 0.7\%), peptidases and peptidase inhibitors (MEROPS DB; 443 orthogroups, $0.4 \%$ ), and transporters (TCDB; 1154, 1.10\%). The total number of gene families with signal peptides was 15,076 (14.4\%), among which 2,869 (2.7\%) were annotated as effectors (Supplementary Table 7). When all ingroup taxa were compared, we observed no significant differences between the number of genes in different functional annotation categories (e.g., CAZymes, transporters, etc.) and ecological mode (i.e., endophytic or non-endophytic; see Supplementary Table 6).

\section{Evolutionary relationships of endophytic, saprotrophic, and pathogenic Xylariaceae s.l. and}

Hypoxylaceae. Maximum likelihood phylogenomic analyses were performed with IQ-Tree using a concatenated matrix of 1,526 universal, single-copy orthogroups (Fig. 1a; Supplementary Fig. 1). Phylogenomic results support the monophyly of the newly proposed families of Graphostromataceae and Hypoxylaceae $^{23}$, as well as previously observed relationships among genera ${ }^{7}$ (Supplementary Fig. 1). Dense gene sampling resulted in improved resolution and statistical support for deeper internal branches compared to a previous five-gene analysis ${ }^{7}$. Inclusion of previously unstudied endophytic taxa markedly increased the known phylogenetic diversity of the family ${ }^{7}$ (Supplementary Fig. 1), highlighting the importance of including unnamed endophytes (which are typically sterile mycelium in culture which precludes morphological characterization and formal naming; but see ${ }^{115}$ ) in phylogenetic studies.

Our analyses revealed seven endophytic isolates in five distinct clades (i.e., clades E2, E4, E5, E6, and E6) nested between the Graphostromataceae and Xylariaceae sensu stricto. To better ascertain their taxonomic relationships, we performed additional phylogenetic analysis that included recently published xylarialean taxa closely related to Xylariaceae and Graphostromataceae (i.e., Barrmaelia, Barrmaeliaceae $^{22}$; Linosporopsis and Clypeosphaeria, Xylariaceae ${ }^{116}$ ). Briefly, we queried sequences of RPB2, alpha-actin, beta-tubulin, and ITS nrDNA for 35 taxa not included in previous multilocus analyses that contained xylarialean endophytes ( ref $^{7}$ ) (e.g., Barrmaelia, Linosporopsis, Clypeosphaeria Entosordaria, Graphostroma; Cryptostroma ${ }^{117}$ ) against the reference multilocus Xylariaceae tree ${ }^{7}$ in Tree-Based Alignment Selector Toolkit (T-BAS) v2.2 ${ }^{118-120}$ with the evolutionary placement algorithm in $\mathrm{RAxML}^{121}$. The settings that we used to place taxa within the reference tree were as follows: UNITE filter off, no clustering, likelihood weights (fast), with the outgroup selected, and data were retained for all 
isolates. This analysis revealed that endophytes in clade E4 are sister to Barrmaelia, endophytes in clade E5 are sister to Linosporopsis, and endophytes in clade E6 are sister to Clypeosphaeria (Supplementary Fig. 1). Thus, our use of Xylariaceae sensu stricto and Xylariaceae sensu lato corresponds to ref ${ }^{22}$ (see Supplementary Fig. 1).

Phylogenomic results are robust to outgroup taxa, gene selection, and model of evolution. To assess the robustness of our phylogenetic results we reconstructed the phylogeny of Xylariaceae s.1. and Hypoxylaceae using four different approaches that differed in either outgroup taxon selection, model of inference, or orthologous gene set. First, we performed a maximum likelihood (ML) analysis of 1,526 single-copy orthogroups (found across all 121 ingroup and outgroup taxa) with the LG model of evolution (Fig. 1, Supplementary Fig. 1). Second, we performed an ML analysis of the same orthologous genes and 121 taxa, but with the JTT+F+I+G4 model of evolution, which was the best evolutionary model selected by ModelFinder in IQ-TREE (Supplementary Fig. 2). Third, we performed an ML analysis with the $\mathrm{JTT}+\mathrm{F}+\mathrm{I}+\mathrm{G} 4$ model of evolution and the same orthologous genes, but after removing non-Xylariales taxa from the outgroup (data not shown). We performed a fourth ML analysis with all taxa (i.e., 121 ingroup and outgroup), but with 1,086 protein sequences identified as universal fungal orthologs with fungal genomes from JGI Mycocosm ${ }^{62}$. JGI orthologs were identified in genomes using the PHYling pipeline (DOI: 10.5281/zenodo.1257002; https://github.com/stajichlab/PHYling unified). All phylogenetic analyses were performed with IQ-TREE multicore v1.6.1178 with 1,000 ultrafast bootstrap replicates (data not shown). All phylogenetic analyses resulted in similar topologies. However, relationships among taxa in the Xylaria HY and E9 clades differed slightly with the LG model (analysis 1) and the $\mathrm{JTT}+\mathrm{F}+\mathrm{I}+\mathrm{G} 4$ (analysis 2) (see Supplementary Fig. 2).

In previous multi-locus analyses, the endophytic isolate Xylariaceae sp. FL2044 was placed as a sister to the Xylariaceae s.l. and Hypoxylaceae ${ }^{7}$. However, our concatenated phylogenomic analyses using different sets of single copy orthologs consistently placed FL2044 as basal within the monophyletic clade containing Xylariaceae s.1. (Fig. 1a; Supplementary Fig. 1). To confirm the placement of FL2044, we computed single-gene trees with IQ-TREE and used ETE Toolkit (http://etetoolkit.org/) to quantify the number of genes that supported the placement of FL2044 as recovered in our concatenated phylogenomic analyses. Overall, of the 882 single-gene trees where the placement of FL2044 was highly supported (i.e., >75\% bootstrap), 297 (33.7\%) agree with the placement of FL2044 in our concatenated analyses (Fig. 1; Supplementary Figs. 1,2). However, the placement of FL2044 in the Xylariaceae s.1. clade also was supported by network analysis of shared orthogroups (Supplementary Fig. 10).

Determination of core, family-specific, clade-specific, and isolate-specific orthogroups and SMGCs. 
To visualize the distribution of orthogroups and SMGCs across taxa, we categorized orthogroups/SMGCs into 10 categories (see Supplementary Tables 3,7). To visualize the relative abundance of these categories across the phylogeny, we combined categories in the following manner for Supplementary Fig. 2e. Core: orthogroups/SMGCs present in all 121 taxa (cat a), as well as orthogroups/SMGCs present in all Xylariaceae s.l. and Hypoxylaceae taxa and in some outgroup taxa (cat c). Family-specific (i.e., Xylariaceae s.l. and Hypoxylaceae specific): orthogroups/SMGCs present in all or some Xylariaceae s.l. and Hypoxylaceae taxa, but absent in outgroup taxa (cat b and cat d). Hypoxylaceae-specific: orthogroups/SMGCs present in all or some Hypoxylaceae taxa, but absent in Xylariaceae s.l. taxa and outgroup taxa (cat e and cat f). Xylariaceae s.1.-specific: orthogroups/SMGCs present in all or some Xylariaceae s.l. taxa, but absent in Hypoxylaceae taxa and outgroup taxa (cat $g$ and cat $h$ ). Isolatespecific: orthogroups/SMGCs found only in a single genome (cat j). Orthogroups/SMGCs that did not fall into any of these categories were defined as "other" (cat i). Examples of the "other" category include orthogroups/SMGCs that are present in some outgroup taxa, as well as some Hypoxylaceae and/or Xylariaceae s.1. taxa. Orthogroups/SMGCs distributions falling in the "other" category may have arisen through HGT, ancestral gene duplication and gene loss, or interspecific hybridization ${ }^{122}$. We found that no orthogroups were both unique to- and universally present in all Xylariaceae s.1. and Hypoxylaceae taxa (Supplementary Table 7d). A single orthogroup (annotated as a putative signaling peptide; OG0009755) was specific to and universally distributed in the Hypoxylaceae clade, but no orthogroups met these criteria for the Xylariaceae s.1. clade.

Overall, ca. $21-37 \%$ of the orthogroups per genome (mean $=27.4 \%)$ represented orthogroups shared by all 121 taxa (i.e., core genes; $\mathrm{n}=2,656$ total) (Supplementary Fig. 2e; Supplementary Table 7). An additional 1,831 orthogroups were present in all Xylariaceae s.l. and Hypoxylaceae and one or more outgroup taxa (Supplementary Table 7d), representing an average of 14-23\% orthologous gene families per genome $($ mean $=18.5 \%$; Supplementary Fig. 2e). Gene families unique to Xylariaceae s.l. and Hypoxylaceae (i.e., absent in the outgroups and present in at least one genome in both Hypoxylaceae and Xylariaceae s.1. clades) represented, on average, ca. 1.6\% of orthogroups per genome (Supplementary Fig. $2 \mathrm{e}$, orange bars). An average of $3.0 \%$ and $3.8 \%$ of orthogroups were unique to Hypoxylaceae or Xylariaceae s.1. taxa, respectively (Supplementary Fig. 2e; Supplementary Table 7d).

Orthogroups unique to a single genome (i.e., dispensable orthogroups) represent ca. 1.4 to $15.6 \%$ of the orthogroups per genome for Xylariaceae s.l. and Hypoxylaceae (Supplementary Fig. 2e). Functional annotation using euKaryotic Orthologous Groups (KOGs) revealed a greater fraction of dispensable orthogroups were predicted to be involved in cellular processes and signaling (i.e., 42.6\%) compared to core orthogroups (27.7\%), including a higher fraction of orthogroups annotated as defense mechanisms and extracellular structures (Supplementary Fig. 13; Supplementary Table 7f). Dispensable 
orthogroups also were more likely than core orthogroups to encode proteins secreted through the general secretory pathway ( $15.0 \%$ vs $2.7 \%$ ), supporting the hypothesis that strain-specific genes may provide ecological adaptations ${ }^{44}$. However, the functions of the majority of dispensable orthogroups remain unknown (i.e., only $20 \%$ had functional annotation vs. $90 \%$ of core orthogroups), similar to results from Dothideomycetes genomes ${ }^{44}$.

Comparison of Hypoxylaceae and Xylariaceae s.l. SMGCs to MIBiG. Although there has been increasing biochemical characterization of metabolites from species of Xylariaceae s.l. and Hypoxylaceae (e.g., terpenes and polyketide compounds ${ }^{10}$ ), fewer studies have linked metabolites to gene clusters. Here, we compared predicted SMGCs to a reference database of known metabolites clusters $\left(\mathrm{MIBiG}^{30}\right)$. Only $25 \%$ of predicted SMGCs ( $\mathrm{n}=1,711$, belonging to 816 cluster families) had BLAST hits to 168 unique $\mathrm{MIBiG}^{30}$ accession numbers (Supplementary Table $3 \mathrm{~b}$ ). The majority of MIBiG hits were classified as PKSI ( 808 hits), terpene synthases (268 hits), and PKS-NRPS hybrids (253 hits). The remaining 382 hits were classified as NRPS, PKS-Other, RiPPS, and Other SMGCs. The average similarity of SMGCs to a MIBiG accession was 54\% (range 13-100\%) (Supplementary Table 3), but 587 xylarialean SMGCs were $100 \%$ similar to $38 \mathrm{MIBiG}$ accessions (Supplementary Table 3).

Similarity to MIBiG is currently defined as the percentage of genes in an SMGC with significant BLAST hits to a known $\mathrm{SMGC}^{39}$, yet similarity can be difficult to assess given the dynamic nature of SMGCs (i.e., frequent gene duplications, gene losses, and $\mathrm{HGT}^{41,123}$ ) and the potential for in silico methods to misidentify cluster boundaries. For example, the griseofulvin cluster of Penicillium aethiopicum is predicted to contain 21 genes, but only core genes Gsf A, I, and G have been experimentally validated ${ }^{40}$. Xylaria taxa, despite lacking 13 genes (GsfR2, GsfK, GsfR1, GsfJ, GsfH and all eight genes of unknown function; Supplementary Fig. 4a), produce detectable levels of griseofulvin in culture (Supplementary Fig. 4b, see also ${ }^{124}$ ). However, lower similarity may also reflect true differences in cluster composition and the production of similar, but distinct metabolites. Variation may also represent null alleles unable to synthesize the metabolite (e.g., aflatoxin in A. flavus $^{125}$ ). Currently, databases such as MIBiG primarily contain metabolites from bioactive fungi with important roles as human or plant pathogens, and increased effort is needed to link metabolites from xylarialean fungi to specific gene clusters.

Correlation between SMGC content and other functional categories. Consistent with the prevalence of SMGCs among clades of fungi known for their saprotrophic ecological roles (e.g., Aspergillus, Penicillium ${ }^{13,14}$ ), we observed that in genomes of non-endophytic Xylariaceae s.l. and Hypoxylaceae SMGC abundance is positively correlated with the number of genes important for saprotrophy (e.g., 
CAZymes, transporters) and putative pathogenicity (e.g., effectors, peptidases), even after accounting for differences among clades and genome sizes $(\mathrm{P}<0.01$; Supplementary Table 6e). Our results are consistent with strong selection for saprotrophs to maintain large gene repertoires to degrade diverse lignocellulosic compounds ${ }^{44}$, as well as highly diverse SMGCs that likely increase competitive abilities in diverse microbial communities ${ }^{11,49,50}$. However, no such correlation was observed for genomes of endophytes in either clade, despite endophytes containing the same fraction of SMGC accessory genes annotated as CAZymes, peptidases, and effectors (but see Hypoxylaceae clade paired comparisons; Supplementary Table 6c).

Intergenic distances, repetitive elements, effectors, and SMGCs. The software BEDTools version 2.29.2 ${ }^{126}$ was used to calculate the distance between adjacent genes (intergenic distance) and the distance between each gene and the closest repetitive element on the 5' and the 3 ' end following ref ${ }^{127}$. Results were visualized using the package 'ggplot2' version 3.3.2 in R and previously published code ${ }^{127}$ (https:/github.com/lambros-f/blumeria_2017). The mean intergenic distance for all Xylariaceae s.1. and Hypoxylaceae genomes was $1,776 \pm 415$ bp. For all genomes, the distribution of intergenic distances followed a normal distribution, except for the genome of Sodiomyces alkalinus, which also displayed an increase in the frequency of genes with an intergenic distance towards 10,000 bp. Repetitive elements occurred more frequently in gene-sparse regions and at the end of contigs (Supplementary Table 2). Since de novo genome assemblers can collapse when reaching a repetitive region larger than the read length itself $^{128}$, we surmise that our genome assemblies may be fragmented because of complex regions rich in repetitive elements.

To identify whether SMGCs and effectors were in regions of the genome with high repeats and sparse gene content, we performed the same calculation of intergenic distances and visualized the locations as a function of gene density and TE location. We observed no significant differences in the density of repetitive elements for effector genes vs. non-effector genes for genomes of Hypoxylaceae or Xylariaceae s.1. taxa (Supplementary Fig. 5). In the majority of Xylariaceae s.1. and Hypoxylaceae genomes, numerous SMGCs, and genes annotated as effectors are located at the edge of contigs in gene sparse/high repeat regions including the griseofulvin cluster in Xylaria. sp. However, there was no relationship between SMGC number (residuals after accounting for genome size) and the number of scaffolds obtained from genome assembly (Supplementary Fig. 16) suggesting that fragmentation of genome assemblies did not artificially increase the predicted number of SMGCs ${ }^{103}$. Repetitive-rich regions, often near telomeres and centromeres, can represent hotspots of gene gain/loss events as transposable elements facilitate gene dispersal both within and among genomes ${ }^{11,17,129}$. The presence of 
SMGCs in these regions may drive the hyperdiversity of SMGCs within the Xylariales, as well as the discontinuous phylogenetic distribution of SMGCs across the studied genomes (see Figs. 1,3).

Confirmation of griseofulvin HGT. We examined regions flanking in Xylaria sp. with and without the griseofulvin cluster to further confirm HGT. Briefly, $30 \mathrm{kbp}$ sequences located up- and downstream of the griseofulvin cluster of Xylaria flabelliformis CBS 123580 were queried with BLASTn against closely related genomes without the griseofulvin cluster to identify homologous regions (X. longipes CBS 148.73 scaffold 57 and $X$. acuta CBS 122032 scaffold 139). Scaffolds containing these homologous regions, along with the scaffolds containing the griseofulvin cluster in X. flabelliformis NC1011 (scaffold 71), $X$. flabelliformis CBS 124033 (scaffold 75), X. flabelliformis CBS 123580 (scaffold 16), X. flabelliformis CBS 114988 (scaffold_56),X. flabelliformis CBS 116.85 (scaffold 29), were then aligned using Mauve $^{130}$. In X. flabelliformis isolates, the scaffold alignment contains the up- and downstream homology blocks with the intervening griseofulvin cluster. Up- and downstream homology blocks were also found in $X$. longipes CBS 148.73; however, the griseofulvin cluster was not present, thus supporting the HGT of griseofulvin cluster in some taxa.

Comparison of leaf litter decomposition among clades. To assess the ability of Xylariaceae s.1. and Hypoxylaceae fungi to degrade lignocellulose, we collected fresh, healthy, green leaf material from two individuals of Quercus virginiana and Pinus halepensis at the University of Arizona campus arboretum. Trees are cultivated in a park-like setting with supplemental water and appear healthy. For both species, leaves were washed in tap water to remove any surface debris. Washed leaves were autoclaved for $20 \mathrm{~min}$ to inactivate endogenous microbes and then dried overnight at $60^{\circ} \mathrm{C}$. Autoclaved leaves $(0.5 \mathrm{~g})$ were placed into individual, sterile $100 \mathrm{~mm}$ Petri plates (three replicate plates per leaf substrate type for each fungal isolate). For each fungal isolate, a $6 \mathrm{~mm}$ plug of mycelium (actively growing on 2\% MEA) was briefly homogenized with a sterile minipestle in $1 \mathrm{~mL}$ of sterile water until mycelia had visually separated from the agar chunks. From this $1 \mathrm{~mL}$ mixture, $75 \mu \mathrm{L}$ was diluted with $3 \mathrm{~mL}$ of sterile water and mixed via pipetting to create the fungal inoculum. One $\mathrm{mL}$ of the diluted ground mycelium was placed directly on the sterile leaf surface in each Petri dish. Negative control samples were inoculated in parallel with sterile water. In total, we inoculated three replicate plates per fungal isolate per plant species (total of 120 plates). Petri plates were sealed with Parafilm and weighed on an analytical balance (mass original $_{\text {) }}$. Plates were stored in the dark at $26^{\circ} \mathrm{C}$ for the duration of the experiment (12 weeks). Each plate was weighed weekly, and the percent of leaf tissue covered with mycelium was visually scored $(0=$ no visible growth; $1=1-25 \%$ leaf coverage; $2=26-50 \%$ leaf coverage; $3=51-75 \%$ leaf coverage; $4=76-100 \%$ leaf coverage). Negative controls did not display fungal growth. We calculated mass loss for each replicate 
and control as $\operatorname{mass}_{\text {final }}=$ mass $_{\text {week } 12}-$ mass $_{\text {original }}$. To account for water loss due to evaporation, we then subtracted the average value of the negative control plates $\left(\operatorname{mass}_{\text {norm }}=\operatorname{mass}_{\text {final }}-\right.$ mass $\left._{\text {control }}\right)$. We compared the normalized mass loss among clades with ANOVA (Supplementary Fig. 11).

Metabolite extraction and identification. To induce the production of SMs and potentially verify SMGCs, we performed co-culture experiments with three isolates: X. flabelliformis NC1011, Xylaria arbuscula FL1030, and Daldinia sp. FL1419. Isolates were grown on Aspergillus defined media ${ }^{131}$. After one week, we removed $6 \mathrm{~mm}$ diameter plugs of actively growing mycelium from each isolate for three pairwise combinations of co-culture plates (i.e., NC1011 vs. FL1419; FL1419 vs. NC1030; NC1030 vs. NC1011). Briefly, agar plugs of two isolates were placed $4.5 \mathrm{~cm}$ apart across the horizontal diameter of a $100 \mathrm{~mm}$ Petri dish (see Supplementary Fig. 4b). We incolulated four replicate co-culture plates for each combination (total 12 interaction plates) and four plates containing each isolate alone (total 12 positive control plates). Plates were incubated at room temperature for 8-10 days or until the mycelium from the two isolates was $\sim 1 \mathrm{~cm}$ apart. Using a sterile transfer tube, we harvested five $6 \mathrm{~mm}$ plugs of agar either (i) next to a single culture (i.e, positive control plates); (ii) in the space between isolates (i.e., interaction plates) to ensure the capture of exogenous SMs; or (iii) in the middle of media control plate. After harvesting agar plugs were placed into sterile, $2.0 \mathrm{~mL}$ microcentrifuge tubes, flash frozen in liquid Nitrogen, and stored at $-80^{\circ} \mathrm{C}$. Frozen samples were shipped to JGI for extraction and stored and $-80^{\circ} \mathrm{C}$ until processed.

To extract metabolites for LC-MS/MS, samples were lyophilized dry (FreeZone 2.5 Plus, Labconco), then bead-beaten to a fine powder with a $3.2 \mathrm{~mm}$ stainless steel bead for 5 seconds $(2 \mathrm{x})$ in a bead-beater (Mini-Beadbeater-96, BioSpec Products). For extraction, $500 \mu \mathrm{L}$ of $\mathrm{MeOH}$ was added to each sample, briefly vortexed, sonicated in a water bath for 5 minutes, and centrifuged for 5 min at 5000 rpm to pellet agar and cellular debris. The supernatant was transferred to a $2 \mathrm{~mL}$ Eppendorf, dried in a SpeedVac (SPD111V, Thermo Scientific), and stored at $-80^{\circ} \mathrm{C}$. Extraction controls were prepared similarly but using empty tubes exposed to the same extraction procedures. In preparation for LC-MS/MS analysis, dried extracts were resuspended by adding $300 \mu \mathrm{L}$ methanol containing $10 \mu \mathrm{g} / \mathrm{mL}$ of 2-Amino3-bromo-5methylbenzoic acid (\#R435902, Sigma) as internal standard, vortexed briefly, sonicated in a water bath for $10 \mathrm{~min}$, and centrifuged ( $5 \mathrm{~min}$ at $5000 \mathrm{rpm}$ ). After centrifugation, $150 \mu \mathrm{L}$ of the resuspended extract was filtered via centrifugation $(2.5 \mathrm{~min}$ at $2500 \mathrm{rpm})$ through a $0.22 \mu \mathrm{m}$ filter (UFC40GV0S, Millipore) and transferred to a glass autosampler vial.

Samples were analyzed on a system consisting of an Agilent 1290 UHPLC coupled to a Thermo QExactive Orbitrap HF (Thermo Scientific, San Jose, CA) mass spectrometer. Reverse phase chromatography was performed by injecting $2 \mu \mathrm{L}$ extract into a C18 column (Agilent ZORBAX Eclipse 
Plus $\mathrm{C} 18,2.1 \times 50 \mathrm{~mm}, 1.8 \mu \mathrm{m}$ ) warmed to $60^{\circ} \mathrm{C}$ with a flow rate of $0.4 \mathrm{~mL} / \mathrm{min}$ equilibrated with $100 \%$ buffer A (100\% LC-MS water with $0.1 \%$ formic acid) for 1 minute, followed by a linear gradient to $100 \%$ buffer B (100\% acetonitrile w/ 0.1\% formic acid) for 7 minutes, and then held at $100 \% \mathrm{~B}$ for 1.5 minutes. MS and MS/MS data were collected in both positive and negative ion mode, with full MS spectra acquired ranging from $90-1350 \mathrm{~m} / \mathrm{z}$ at 60,000 resolution, and fragmentation data acquired using an average of stepped collision energies of 10,20 and $40 \mathrm{eV}$ at 17,500 resolution. Orbitrap instrument parameters included a sheath gas flow rate of 50 (au), an auxiliary gas flow rate of 20 (au), sweep gas flow rate of $2(\mathrm{au}), 3 \mathrm{kV}$ spray voltage, and $400^{\circ} \mathrm{C}$ capillary temperature. Sample injection order was randomized and an injection blank of methanol only run between each sample. Metabolites were identified based on comparing exact mass (ppm difference between detected $\mathrm{m} / \mathrm{z}$ to a compound's theoretical $\mathrm{m} / \mathrm{z}$ ) and comparing experimental MS/MS fragmentation spectra to that of standards. These data confirmed the production of griseofulvin by NC1011 when grown in co-culture with FL1419 (Supplementary Fig. 4b).

\section{SUPPLEMENTARY REFERENCES}

115. Harrington, A. H. et al. Coniochaeta endophytica sp. nov., a foliar endophyte associated with healthy photosynthetic tissue of Platycladus orientalis (Cupressaceae). Plant and Fungal Systematics 64, 65-79 (2019).

116. Voglmayr, H. \& Beenken, L. Linosporopsis, a new leaf-inhabiting scolecosporous genus in Xylariaceae. Mycol. Prog. 19, 205-222 (2020).

117. Li, Q. et al. Phylogeny of Graphostromatacea with three species isolated in China. Research Square (2021) doi:10.21203/rs.3.rs-398791/v1.

118. Miller, M. A. et al. A RESTful API for Access to Phylogenetic Tools via the CIPRES Science Gateway. Evol. Bioinform. Online 11, 43-48 (2015).

119. Carbone, I. et al. T-BAS Version 2.1: Tree-based alignment selector toolkit for evolutionary placement of DNA sequences and viewing alignments and specimen metadata on curated and custom trees. Microbiol Resour Announc 8, (2019).

120. Carbone, I. et al. T-BAS: Tree-Based Alignment Selector toolkit for phylogenetic-based placement, alignment downloads and metadata visualization: an example with the Pezizomycotina tree of life. Bioinformatics 33, 1160-1168 (2017). 
121. Berger, S. A., Krompass, D. \& Stamatakis, A. Performance, accuracy, and Web server for evolutionary placement of short sequence reads under maximum likelihood. Syst. Biol. 60, 291-302 (2011).

122. Keeling, P. J. \& Palmer, J. D. Horizontal gene transfer in eukaryotic evolution. Nat. Rev. Genet. 9, 605-618 (2008).

123. Lind, A. L. et al. Drivers of genetic diversity in secondary metabolic gene clusters within a fungal species. PLoS Biol. 15, e2003583 (2017).

124. Mead, M. E. et al. Draft genome sequence of the griseofulvin-producing fungus Xylaria flabelliformis Strain G536. Microbiol Resour Announc 8, (2019).

125. Chang, P.-K., Horn, B. W. \& Dorner, J. W. Sequence breakpoints in the aflatoxin biosynthesis gene cluster and flanking regions in nonaflatoxigenic Aspergillus flavus isolates. Fungal Genet. Biol. 42, 914-923 (2005).

126. Quinlan, A. R. BEDTools: the Swiss-army tool for genome feature analysis. Curr. Protoc. Bioinformatics 47, 11-12 (2014).

127. Frantzeskakis, L. et al. Signatures of host specialization and a recent transposable element burst in the dynamic one-speed genome of the fungal barley powdery mildew pathogen. BMC Genomics $\mathbf{1 9}$, 381 (2018).

128. Thomma, B. P. H. J. et al. Mind the gap; seven reasons to close fragmented genome assemblies. Fungal Genet. Biol. 90, 24-30 (2016).

129. Wisecaver, J. H. \& Rokas, A. Fungal metabolic gene clusters — caravans traveling across genomes and environments. Front. Microbiol. (2015).

130. Darling, A. C. E., Mau, B., Blattner, F. R. \& Perna, N. T. Mauve: multiple alignment of conserved genomic sequence with rearrangements. Genome Res. 14, 1394-1403 (2004).

131. Tucker, S. L. \& Orbach, M. J. Agrobacterium-mediated transformation to create an insertion library in Magnaporthe grisea. Methods Mol. Biol. 354, 57-68 (2007).

132. Virtanen, P. et al. SciPy 1.0: fundamental algorithms for scientific computing in Python. Nat. 
Methods 17, 261-272 (2020).

133. Subramanian, B., Gao, S., Lercher, M. J., Hu, S. \& Chen, W.-H. Evolview v3: a webserver for visualization, annotation, and management of phylogenetic trees. Nucleic Acids Res. 47, W270W275 (2019).

\section{Supplementary Tables}

Supplementary Table 1. (a) Information for the 121 genomes included in this study; (b) Genome and assembly information for 96 Xylariaceae s.l. and Hypoxylaceae genomes included in this study.

Supplementary Table 2. RepeatMasker, RepeatScout, and RepBase Update classification of repetitive elements for 96 genomes of Xylariaceae s.1. and Hypoxylaceae.

Supplementary Table 3. (a) Secondary metabolite gene cluster (SMGC) annotations for the 121 genomes included in this study (according to antiSMASH) and grouped into families with BiGSCAPE; (b) Distribution and percent similarity of Xylariaceae s.l. and Hypoxylacae SMGCs to 168 MIBiG accessions; (c) Count and percentage of all SMGCs and SMGC families per category (A-J); (d-j) Count and percentage of SMGCs per type (e.g., NRPS, Terpene, Other PKS, PKS-NRP Hybrids, Other, RiPP) per category (A-J).

Supplementary Table 4. (a) Count of catabolic gene clusters (CGCs) by anchor gene; (b) Presence/Absence of CGC families per genome; (c) Composition of the CGC families; (d) Genomic position and annotation of CGCs.

Supplementary Table 5. (a) Taxonomic and phylogenetic information for 4,262 putative HGT candidate genes identified by Alien Index (AI); (b) Manual curation of phylogenetic trees reveals 168 HGT candidates (each row is a unique transfer event; orthogroups may appear more than once); (c) Distribution of HGT counts per genome (HGT001-HGT-129 are high confidence transfers and HGT130-HGT290 are ambiguous transfers); (d) Functional annotation of 1,148 SMGC genes identified by the second Alien Index as candidate HGTs.

Supplementary Table 6. (a) Number of genes annotated as MEROPS, CAZymes, PCWDCs, SMGCs, CGCs, and putative HGTs for genomes of 96 Xylariaceae s.l. and Hypoxylaceae; (b) Statistical comparison between Xylariaceae s.1. and Hypoxylaceae genomes; (c) Statistical comparison between endophytic and non-endophytic genomes with phylogenetic independent contrasts (PICS); (d) Statistical analysis of genomic features for paired endophyte/non-endophyte sister taxa using least-squares means contrasts; (e) Pearson correlation of genomic features as a function of ecological mode and clade.

Supplementary Table 7. (a) Orthogroup summary statistics; (b) Orthogroup annotations; (c) Count and percentage of orthogroups and proteins per orthogroup category (A-J). (d) Orthogroups that comprise each category (A-J). 
Additional Files (Available on FigShare Repository; DOI 10.6084/m9.figshare.c.5314025)

Additional file 1. InterProScan annotations for 96 Xylariaceae s.l. and Hypoxylaceae genomes.

Additional file 2. AntiSMASH output for the 96 Hypoxylaceae and Xylariaceae s.l genomes.

Additional file 3. Tables summarizing the ancestral gene reconstruction by Count v10.04. The ancestral gene content was reconstructed for the entire data set, as well as for subsets of orthologous gene families corresponding to different functional groups including (i) CAZymes; (ii) plant cell wall degrading CAZymes (PCWDCs); (iii) PCWDCs involved in the degradation of cellulose, hemicellulose, lignin, pectin, starch and inulin; (iv) peptidases; (v) peptidase inhibitors; (vi) transporters; (vii) transporters involved in the exchange of carbohydrates; (viii) transporters involved in the exchange of amino acids; (ix) transporters involved in the exchange of lipids; (x) transporters involved in the exchange of nitrogen; and (xi) effectors.

Additional file 4. Graphs of intergenic distances for each genome of Xylariaceae s.1. and Hypoxylaceae, overlaid with the location of secondary metabolite gene clusters, repeat elements, and effector genes.

Additional file 5. Graphs depicting the frequency of repetitive elements surrounding genes for each genome of Xylariaceae s.l. and Hypoxylaceae.

Additional file 6. Phylogenomic trees inferred by maximum-likelihood under the $\mathrm{JTT}+\mathrm{F}+\mathrm{I}+\mathrm{G} 4$ model for (a) the whole dataset of 121 taxa and 1,526 protein sequences; (b) a subset of Xylariales taxa only and 1,526 protein sequences; and (c) the entire dataset of 121 taxa and 1,086 protein sequences.

Additional file 7. Table showing the sister clades to Xylariaceae sp. FL2044 recovered by the phylogenetic analysis of each of the 1,526 single-copy orthologous genes.

Additional file 8. Alignment of regions flanking the griseofulvin cluster in Xylaria sp. (a) Mauve ${ }^{130}$ alignment of the scaffolds containing the griseofulvin cluster in X. flabelliformis NC1011, $X$. flabelliformis CBS 124033, X. flabelliformis CBS 123580, X. flabelliformis CBS 114988, X. flabelliformis CBS 116.85, and scaffolds of the closely related Xylaria longipes CBS 148.73 and Xylaria acuta CBS 122032 showing similarity to the griseofulvin flanking regions of $X$. flabelliformis CBS 123580. (b) Same alignment after hiding the scaffolds of Xylaria acuta CBS 122032, X. flabelliformis CBS 124033, X. flabelliformis NC1011, X. flabelliformis CBS 114988. Locally collinear blocks are shown in the same colors. The plot inside the blocks indicates the level of sequence similarity. The ruler above each scaffold represents the nucleotide positions. The white boxes below represent coding sequences. The griseofulvin cluster is highlighted in light blue for $X$. flabelliformis CBS 123580; the purple block contains the griseofulvin protocluster. 
bioRxiv preprint doi: https://doi.org/10.1101/2021.06.01.446356; this version posted June 2, 2021. The copyright holder for this preprint (which was not certified by peer review) is the author/funder, who has granted bioRxiv a license to display the preprint in perpetuity. It is made available under aCC-BY-NC-ND 4.0 International license.

\section{Supplementary Figures}


bioRxiv preprint doi: https://doi.org/10.1101/2021.06.01.446356; this version posted June 2, 2021. The copyright holder for this preprint (which was not certified by peer review) is the author/funder, who has granted bioRxiv a license to display the preprint in perpetuity. It is made available under aCC-BY-NC-ND 4.0 International license.

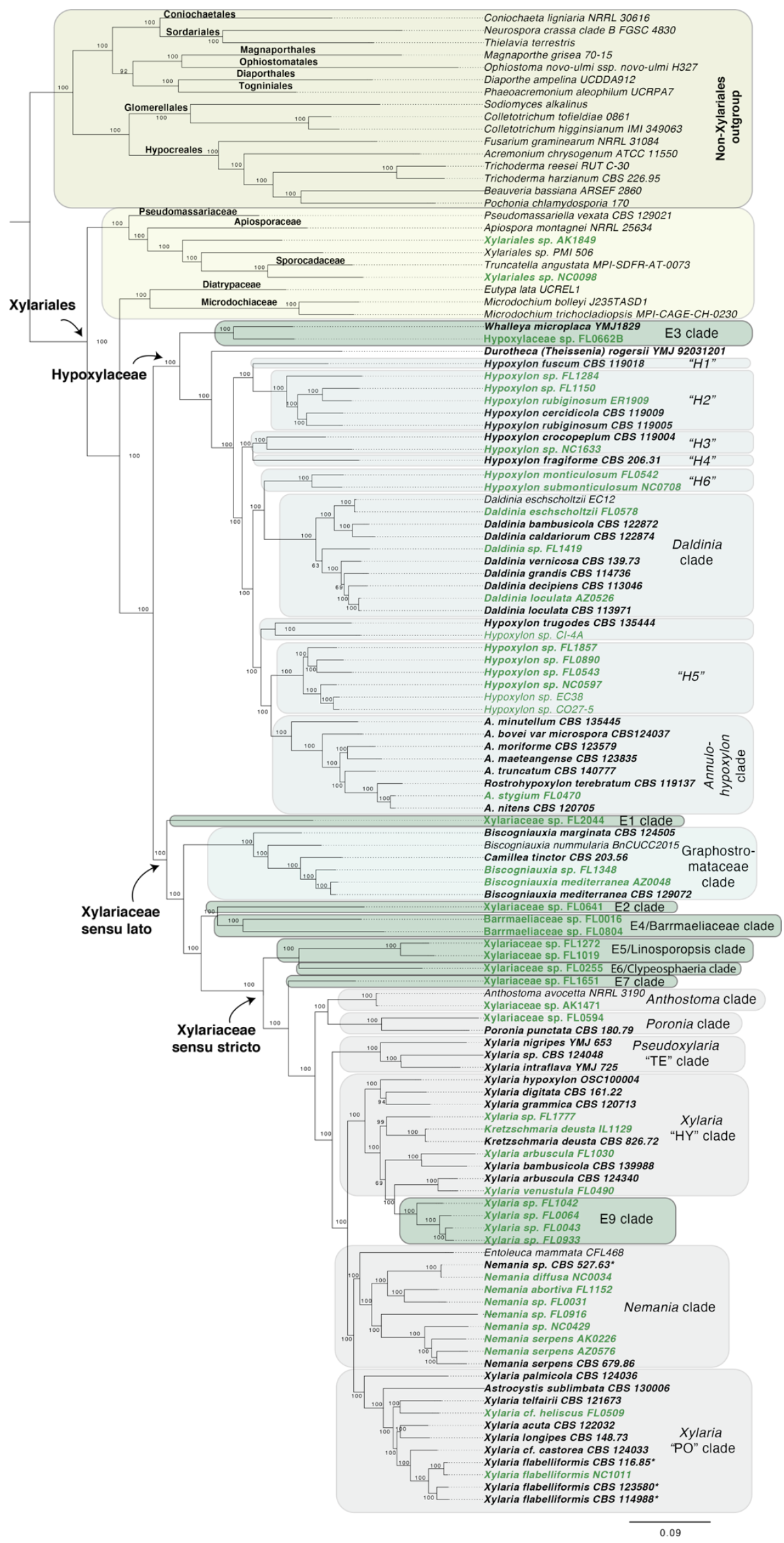


Supplementary Figure 1. Phylogenomic tree inferred by maximum likelihood based on a combination of 1,526 universal single-copy orthologous protein sequences. Twenty-five Sordariomycetes species outside Xylariales were used as the outgroup (Supplementary Table 1a). Isolates sequenced in this study are highlighted in bold. Endophytes (i.e., fungi isolated from living, photosynthetic tissues of plants and lichens ${ }^{7}$ ) are indicated in green. Clade information is based on previously published studies (see refs $7,22,23,42,43)$. Numbers at nodes indicate ultrafast bootstrap support values from IQ-TREE ${ }^{98}$. The scale bar corresponds to the number of substitutions per site. 
a

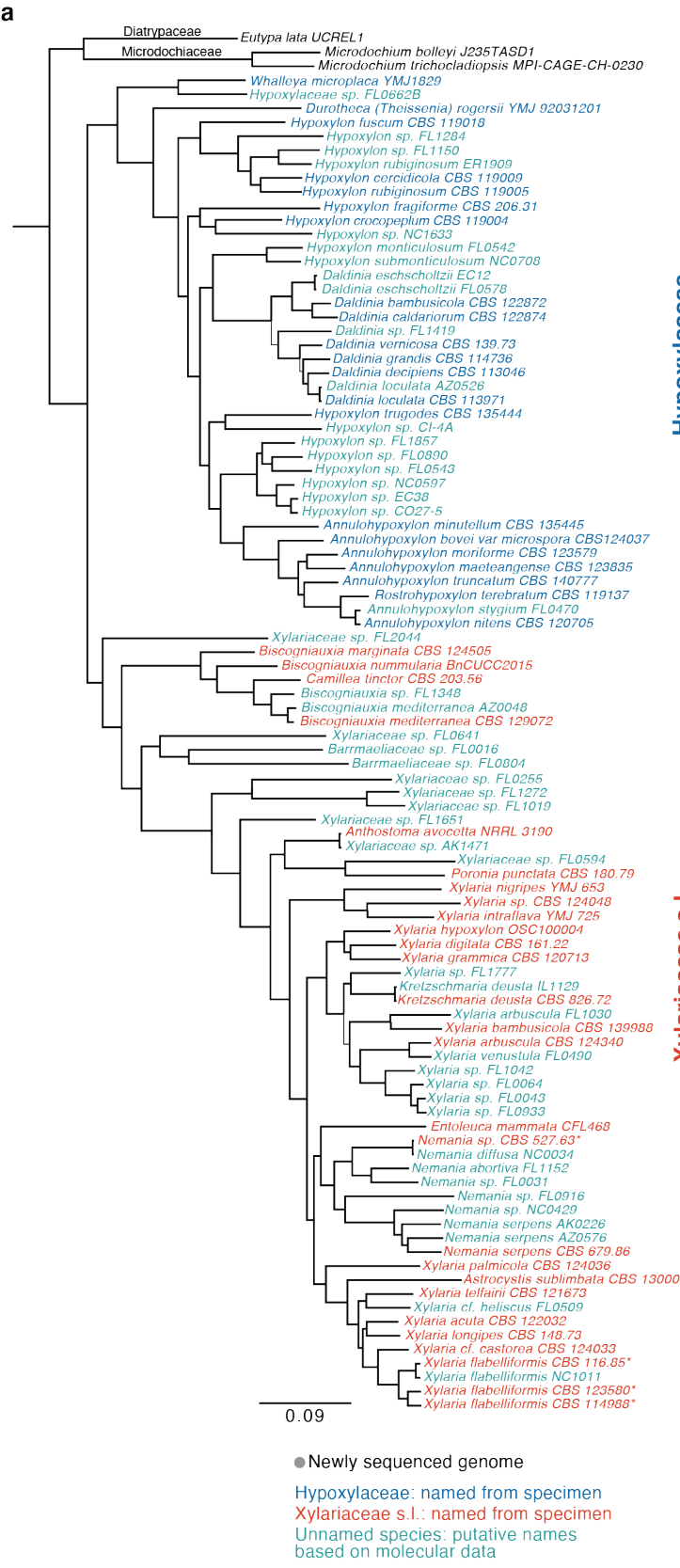

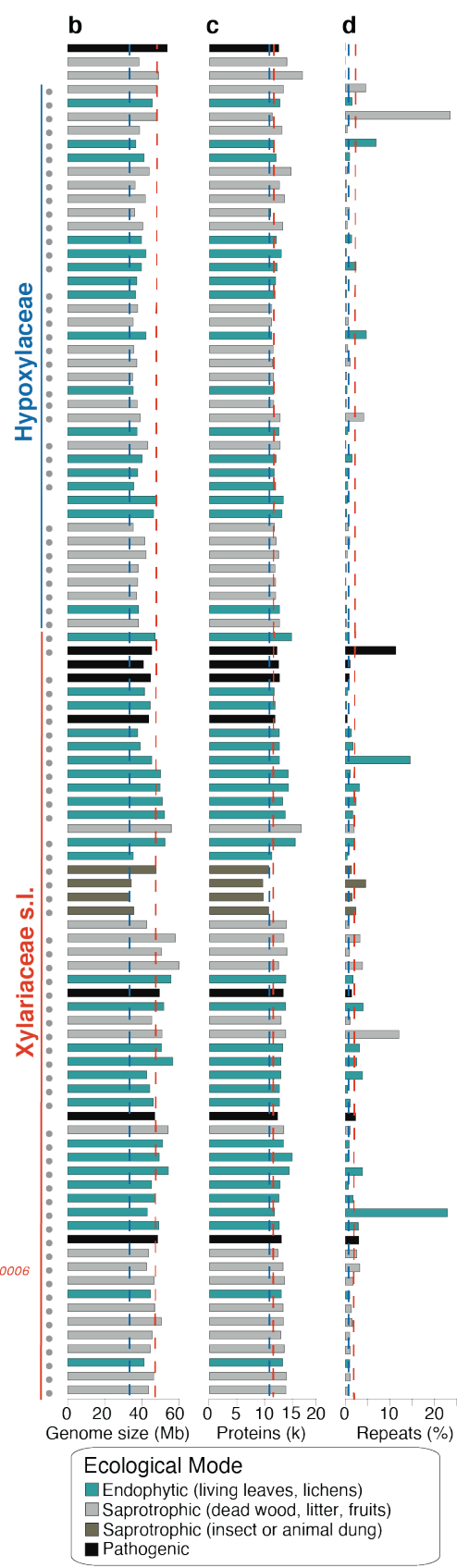

e
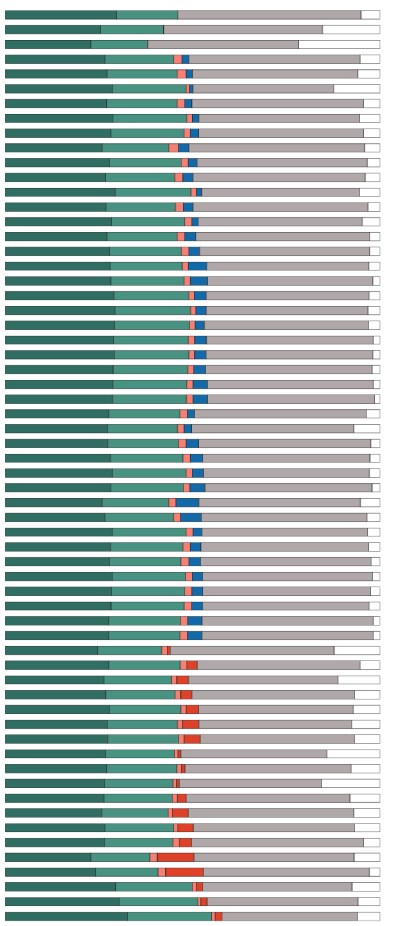

E.rin
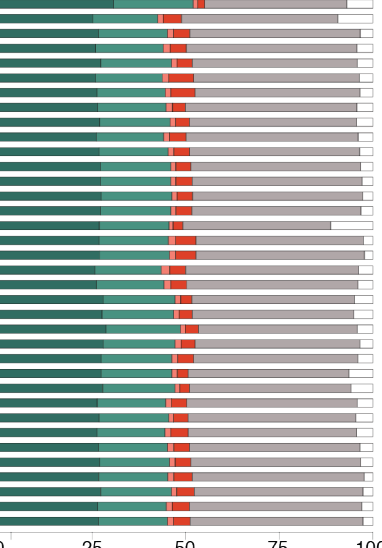

$25 \quad 50 \quad 75$

100

Orthogroup Distribution

All genomes (i.e., core)

Xylariaceae/Hypoxylaceae + some outgroup $\square$ Other

Hypoxylaceae $\square$ Xylariaceae/Hypoxylaceae

Xylariaceae s.I. $\square$ Isolate

Supplementary Figure 2. Phylogenomic reconstruction of Xylariaceae s.l. and Hypoxylaceae and genome statistics. (a) The maximum likelihood phylogram is based on 1,526 single-copy orthologous genes present in all genomes. Bootstrap values are shown in Supplementary Fig. 1. The scale bar indicates the number of substitutions per site. Names of reference taxa are colored according to their clade affiliation (dark blue: Hypoxylaceae; red: Xylariaceae s.l.). Undescribed endophyte species, putatively named based on phylogenetic analyses ${ }^{7}$, are shown in teal blue; (b) genome size; (c) predicted protein coding genes; and (d) percent transposable element (TE) content (bar colors correspond to ecological mode; see legend). Averages per major clade are shown with dotted lines in panels a-d; (e) relative abundance of core, family-specific, clade-specific, and isolate-specific orthogroups (see legend; Supplementary Table 3d). 
a

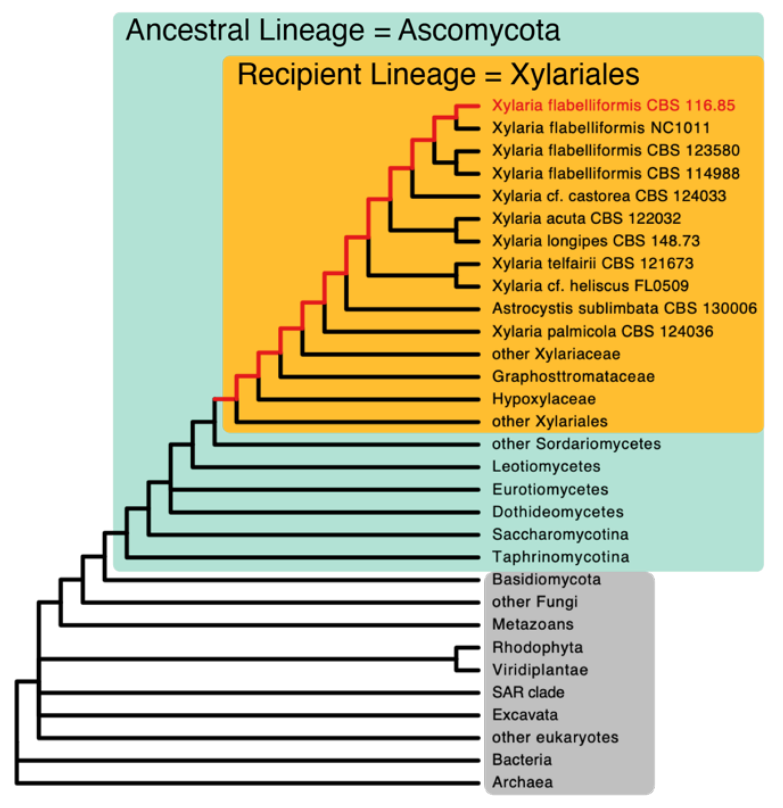

b

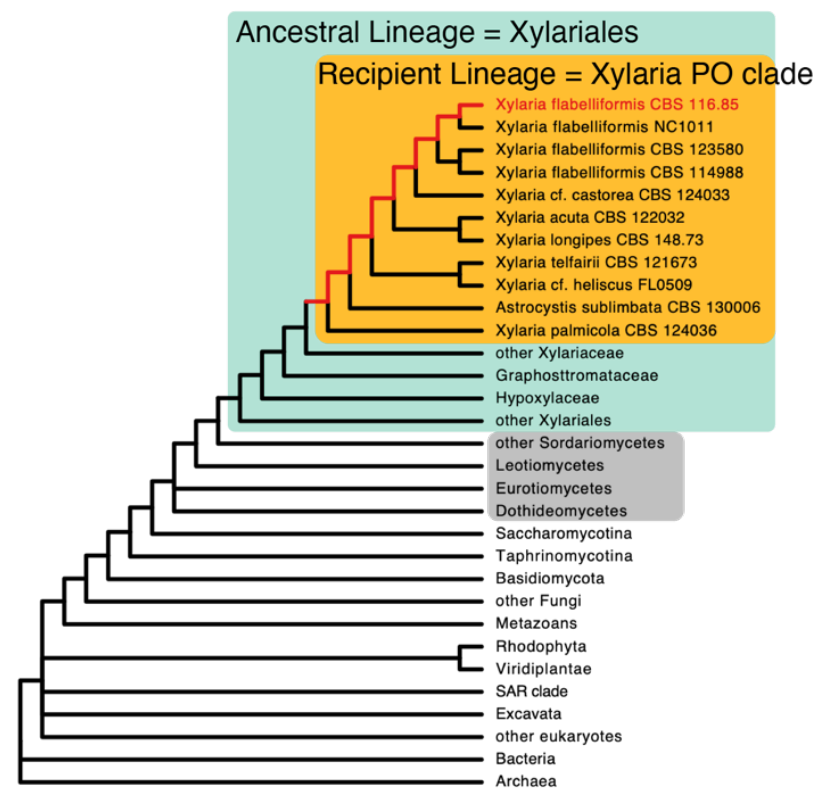

Supplementary Figure 3. Overview of Alien Index (AI) calculations to identify HGT. In this example, Xylaria flabelliformis CBS 116.85 is the query genome. (a) AI screen to identify HGT candidates from more distant gene donors (grey box); candidates must have a better hit to sequences outside the ancestral lineage (Ascomycota; green box). By skipping all sequences to other Xylariales (orange box), HGT candidates could have been acquired at any point back to their last common ancestor (red branches) (b) AI screen to identify more recently acquired HGT candidates from other filamentous fungi (grey box). For this screen, candidates must have a better hit to sequences outside the Xylariales (green box). All sequences to other Xylaria "PO" clade were skipped (orange box) to identify shared HGT candidates acquired at any point back to the last common ancestor of the clade (red branches). 
a

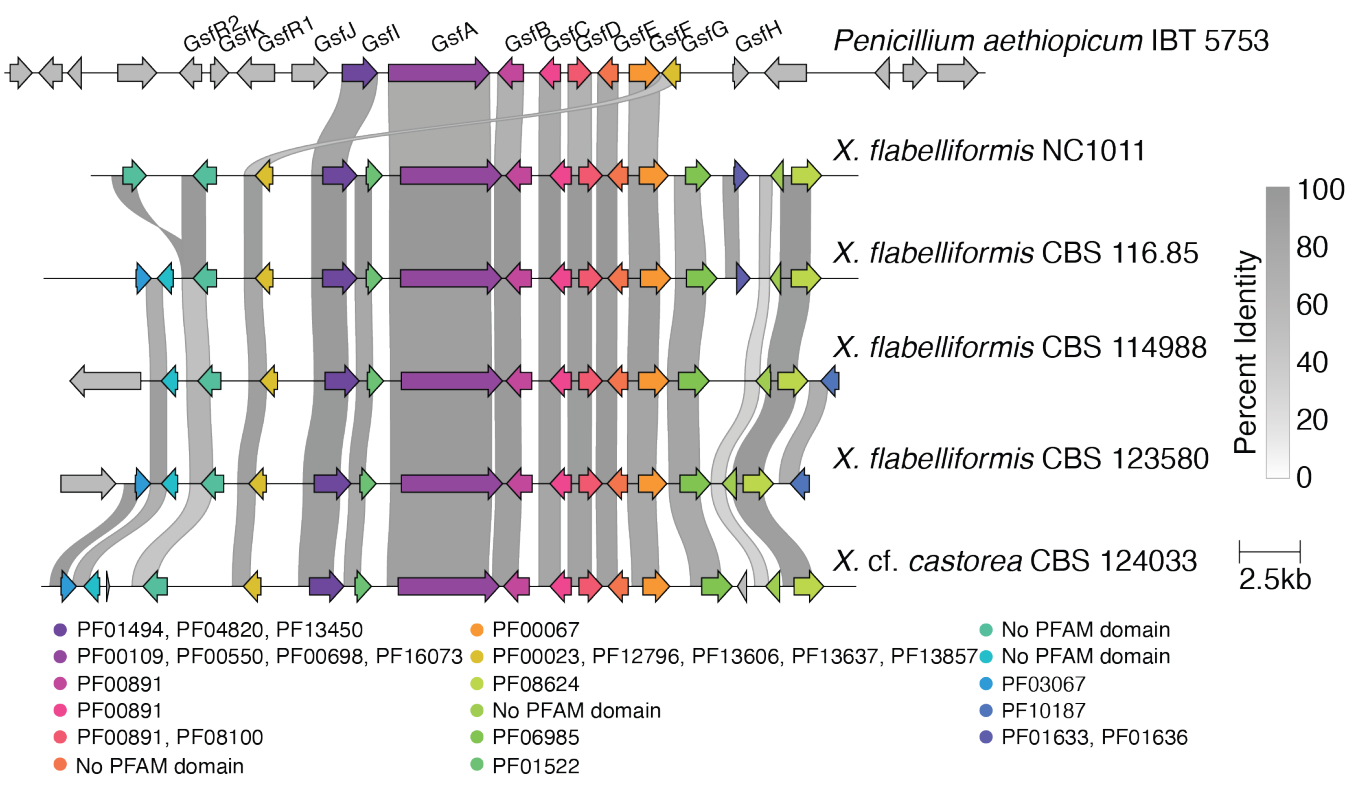

b

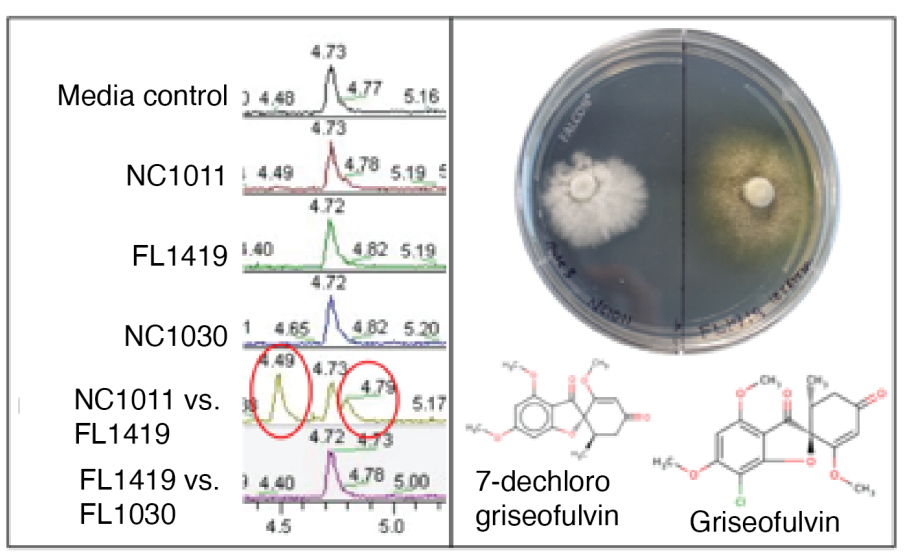

Supplementary Figure 4. Similarity of the griseofulvin SMGC in Penicillium and Xylaria supports HGT. (a) Comparison of the griseofulvin cluster from Penicillium aethiopicum IBT 5753 (top) to five newly sequenced Xylaria genomes. Homologous genes are colored by PFAM domain. Connecting ribbons indicate percent amino acid identity to genes in the Penicillium cluster; (b) Metabolomic analysis of pairwise comparisons of X. flabelliformis NC1011, Xylaria arbuscula FL1030, and Daldinia sp. FL1419 illustrates production of griseofulvin by NC1011 during the interaction with FL1419, but not when grown alone or with isolate FL1030. 


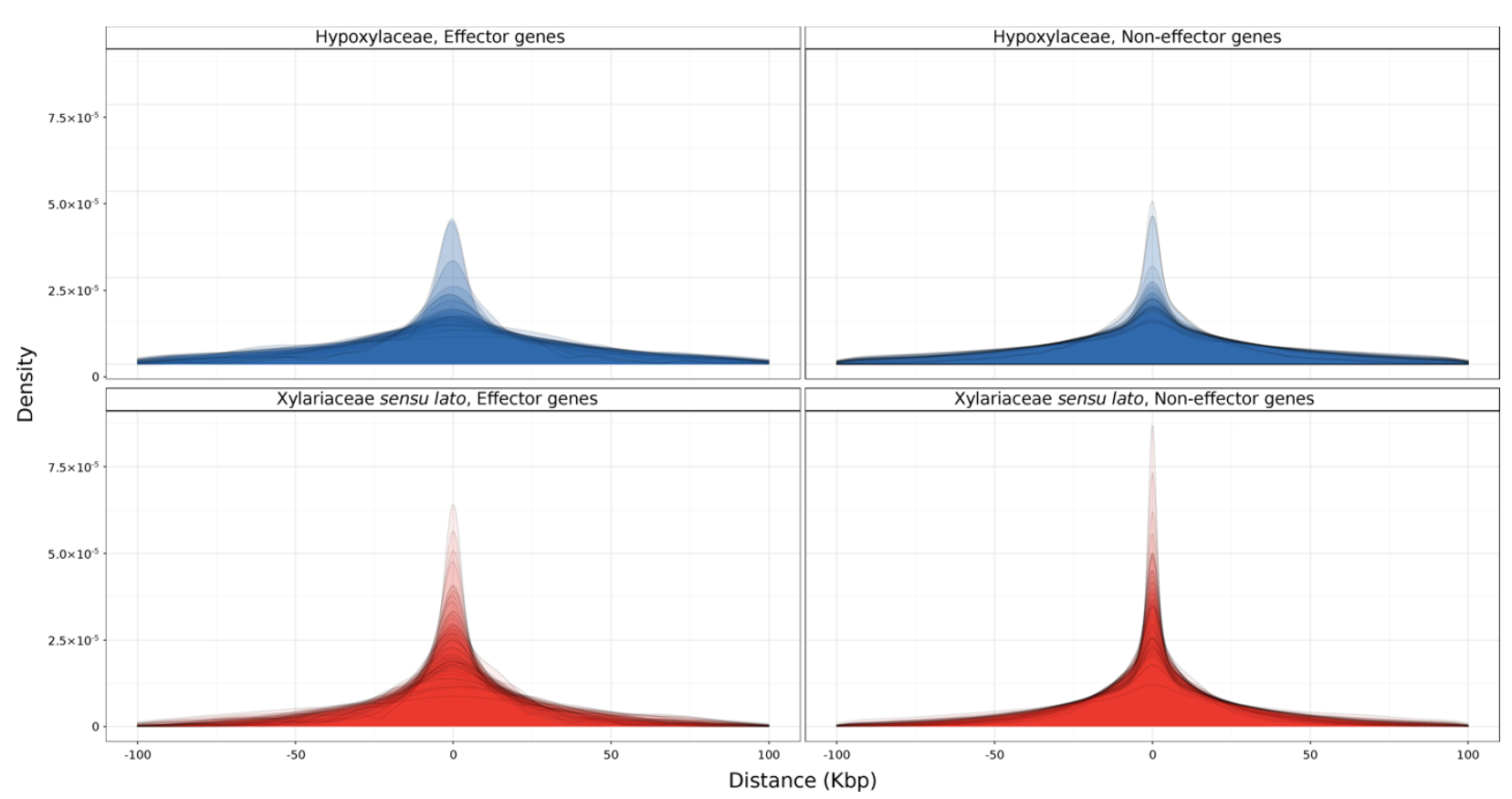

Supplementary Figure 5. The density of repetitive elements surrounding genes was higher for Xylariaceae s.I. than for Hypoxylaceae genomes. Overlapped density plot of taxa per clade, illustrating the distance of repetitive elements from genes annotated as Effector (left) vs. Non-Effector genes (right). Negative distances indicate repetitive elements are located upstream of genes, while positive distances indicate repetitive elements downstream of the genes. Repetitive elements were identified by RepeatScout and RepeatMasker. Effector genes were predicted by EffectorP 2.0. The distances were computed using BEDTools v2.29.2. 

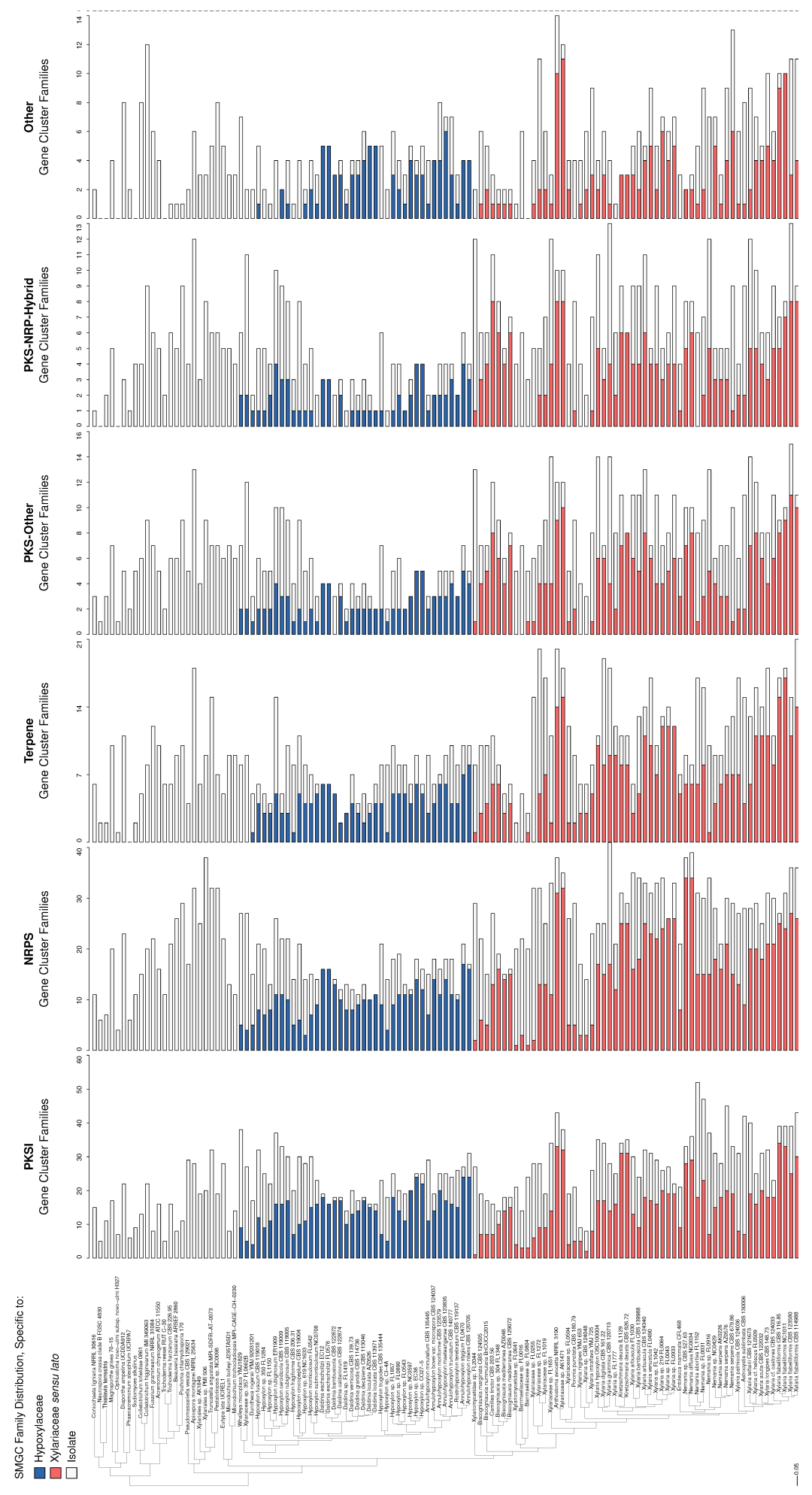

Supplementary Figure 6. The majority of SMGCs are specific to Hypoxylaceae or Xylariaceae s.l. clades or individual isolates regardless of SMGC type. Phylogenomic tree of Xylariaceae s.l. and Hypoxylaceae and outgroup taxa with bar plots illustrating the number of SMGC families per genome, as well as the percentage of clade-specific and isolate-specific SMGC families for (a) PKSI; (b) NRPS; (c) Terpene; (d) PKS-Other; (e) PKS-NRP Hybrid; and (f) Other. 
a

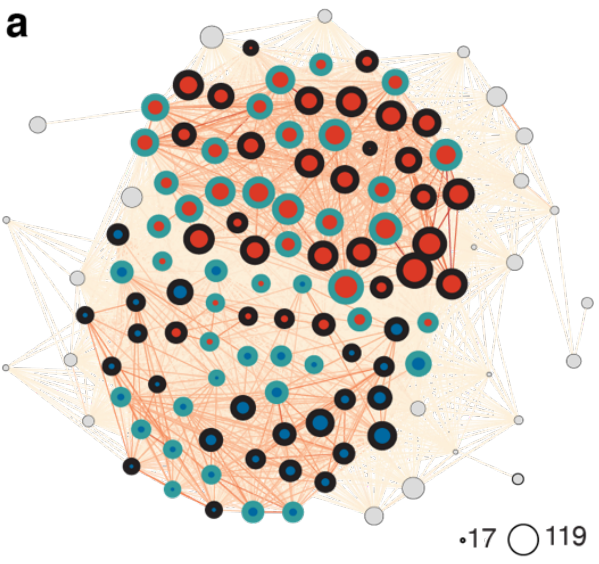

d

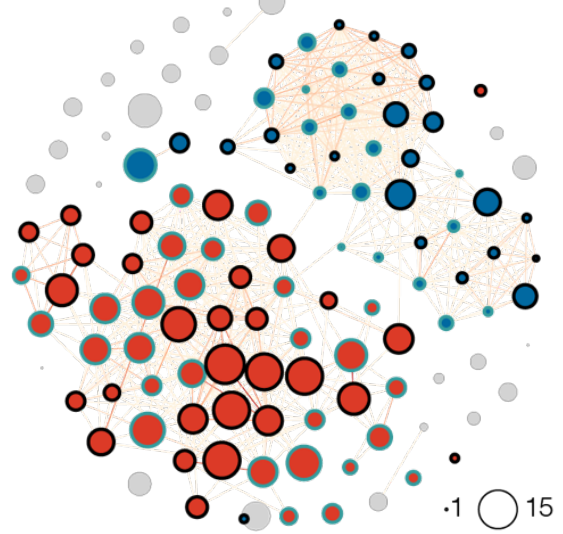

g

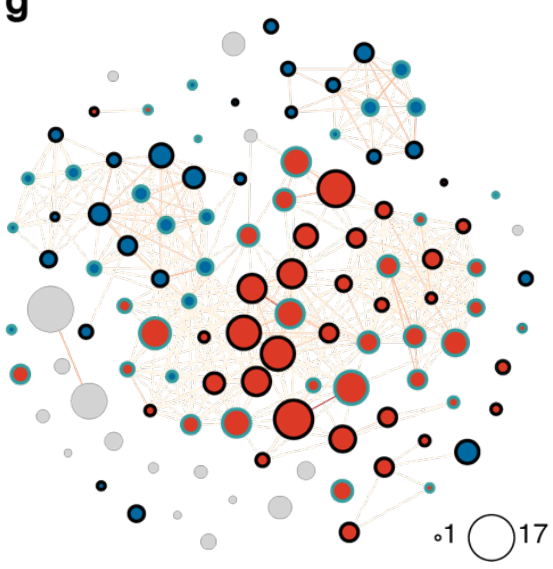

b

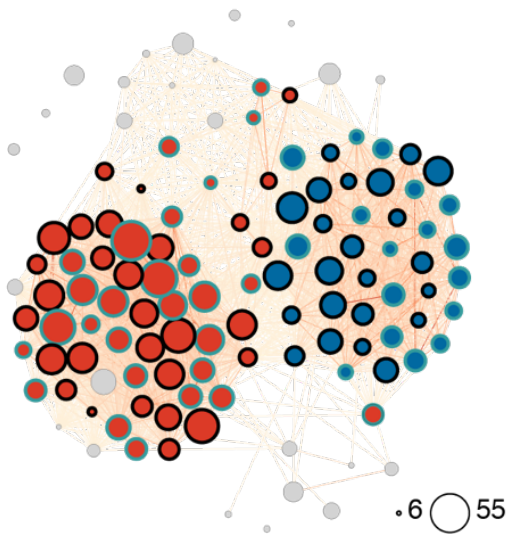

e

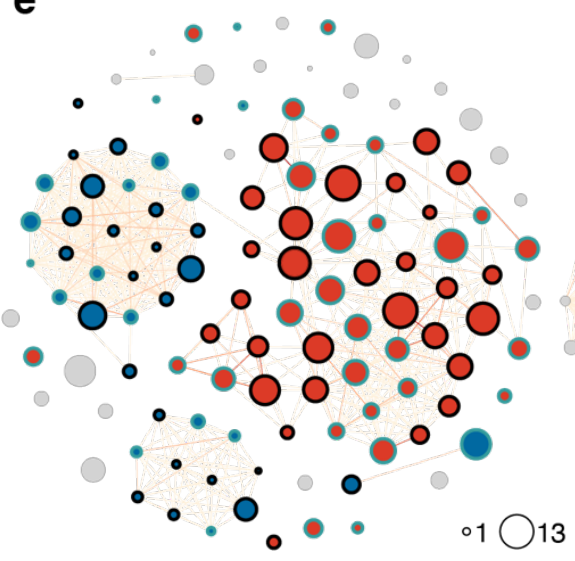

h

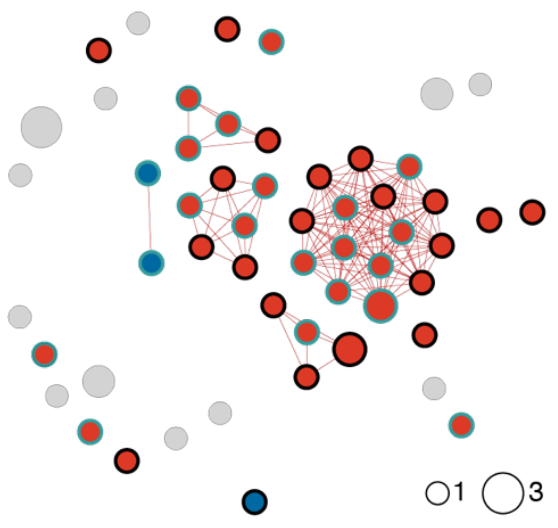

C

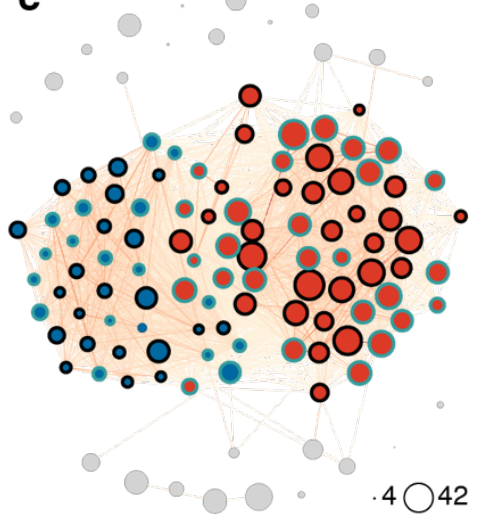

f

Nodes: Clade
Hypoxylaceae Xylariaceae s.I.
Outgroup

Node Borders: Ecological mode Endophyte

O Non-endophyte

Edges: Shared Cluster Families $(-) \square(+)$

Supplementary Figure 7. Network analysis illustrates the importance of clade rather than ecological mode for SMGC content. Network representation of SMGCs clustering from BiG-SCAPE. Each node represents the SMGC content per genome for (a) all SMGCs and SMGC sub-types: (b) PKSI; (c) NRPS; (d) PKS other; (e) PKS-NRPS Hybrids; (f) terpenes; (g) other; and (h) RiPPs. Networks are scaled by the count of gene clusters and positioned by a force-directed layout algorithm. Edges between two nodes are weighted by the number of shared clusters. Node color corresponds to clade. Nodes representing endophytic isolates are shown with blue borders. 


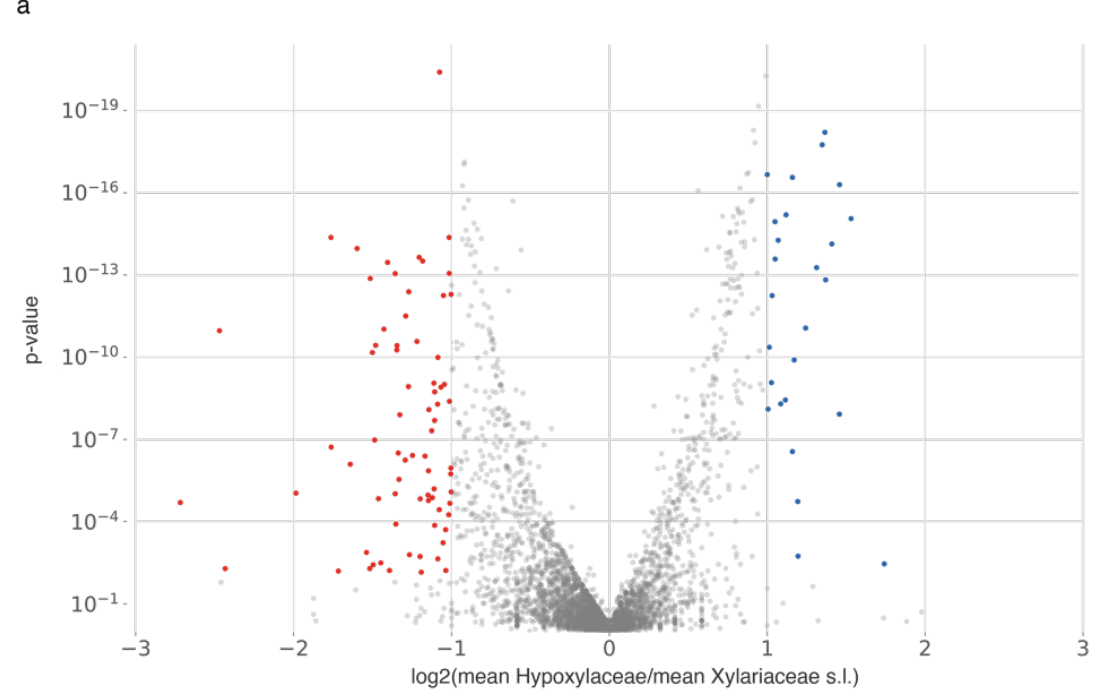

b

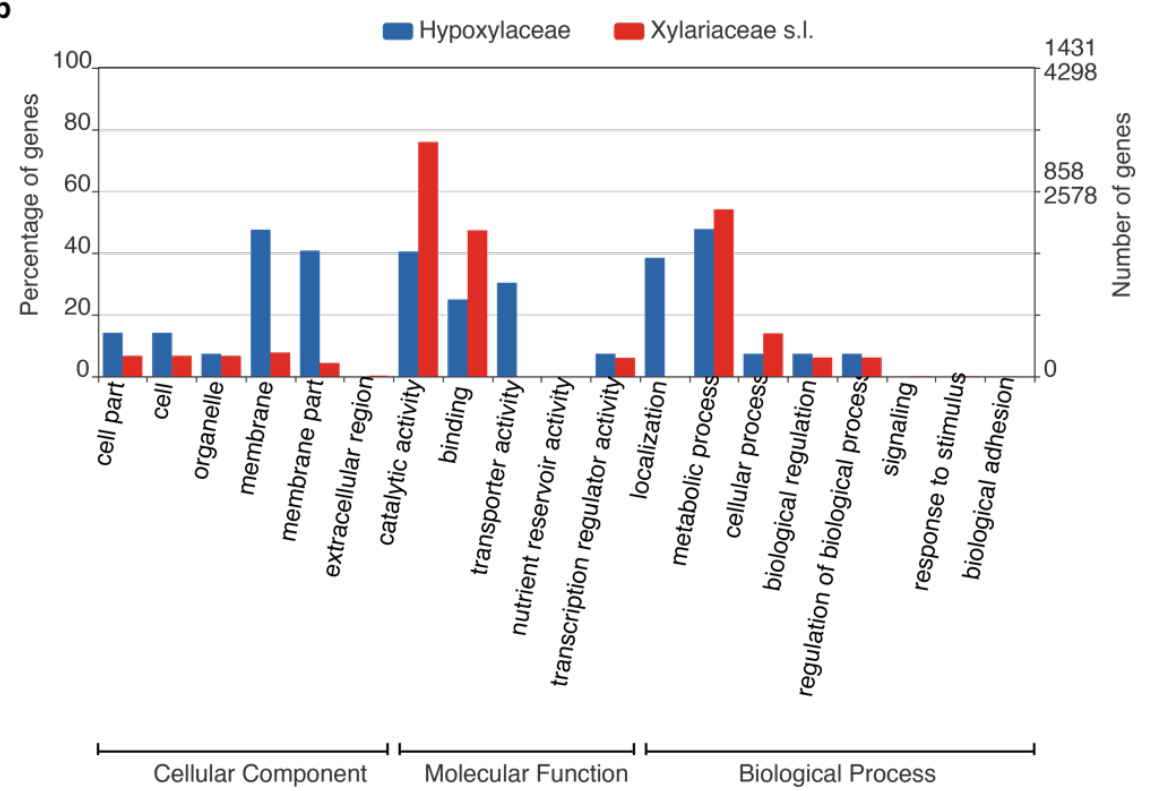

Supplementary Figure 8. Twenty-six orthogroups were significantly enriched in the Hypoxylaceae clade, while 74 orthogroups were significantly expanded in the Xylariaceae s.l. clade. (a) Volcano plot of the protein count representation tests for orthogroups shared between the Hypoxylaceae and Xylariaceae s.1. clades. Orthogroups significantly enriched in Xylariaceae s.l. taxa are colored in red, while orthogroups significantly enriched in Hypoxylaceae taxa are colored in blue. Two-sided MannWhitney U-tests, p-value $\leq 0.01$ and $|\log 2 \mathrm{FC}| \geq 1$. (b) Comparison of enriched GO terms (level 2) of orthogroups significantly enriched in Hypoxylaceae taxa (blue) vs. Xylariaceae s.l. taxa (red). GO terms were analyzed and visualized using Web Gene Ontology Annotation Plot 2.0 (WEGO). See also Supplementary Table $3 \mathrm{f}$ for KOG annotation of enriched orthologs. The two-sided Mann-Whitney U-test was performed using $\mathrm{SciPy}^{132}$ through KinFin v1.099. 

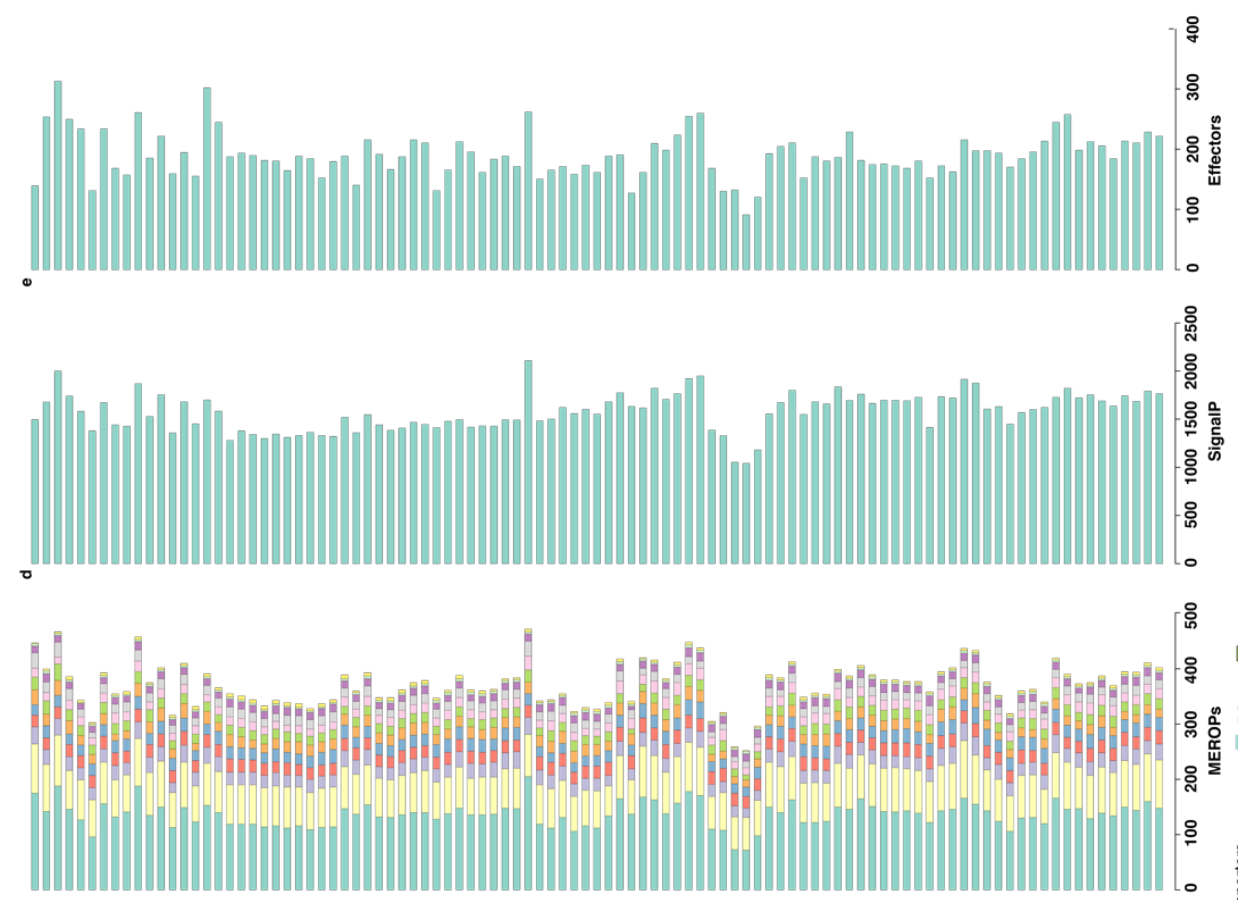

○

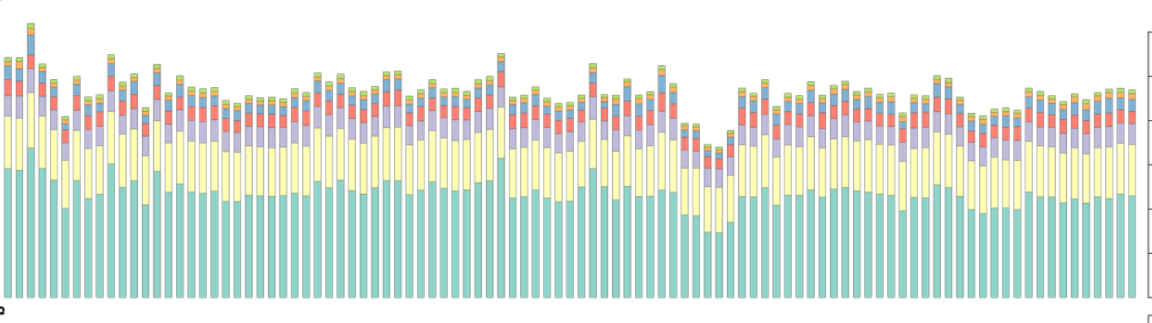

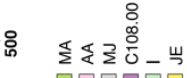

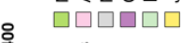

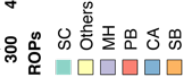

월

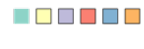
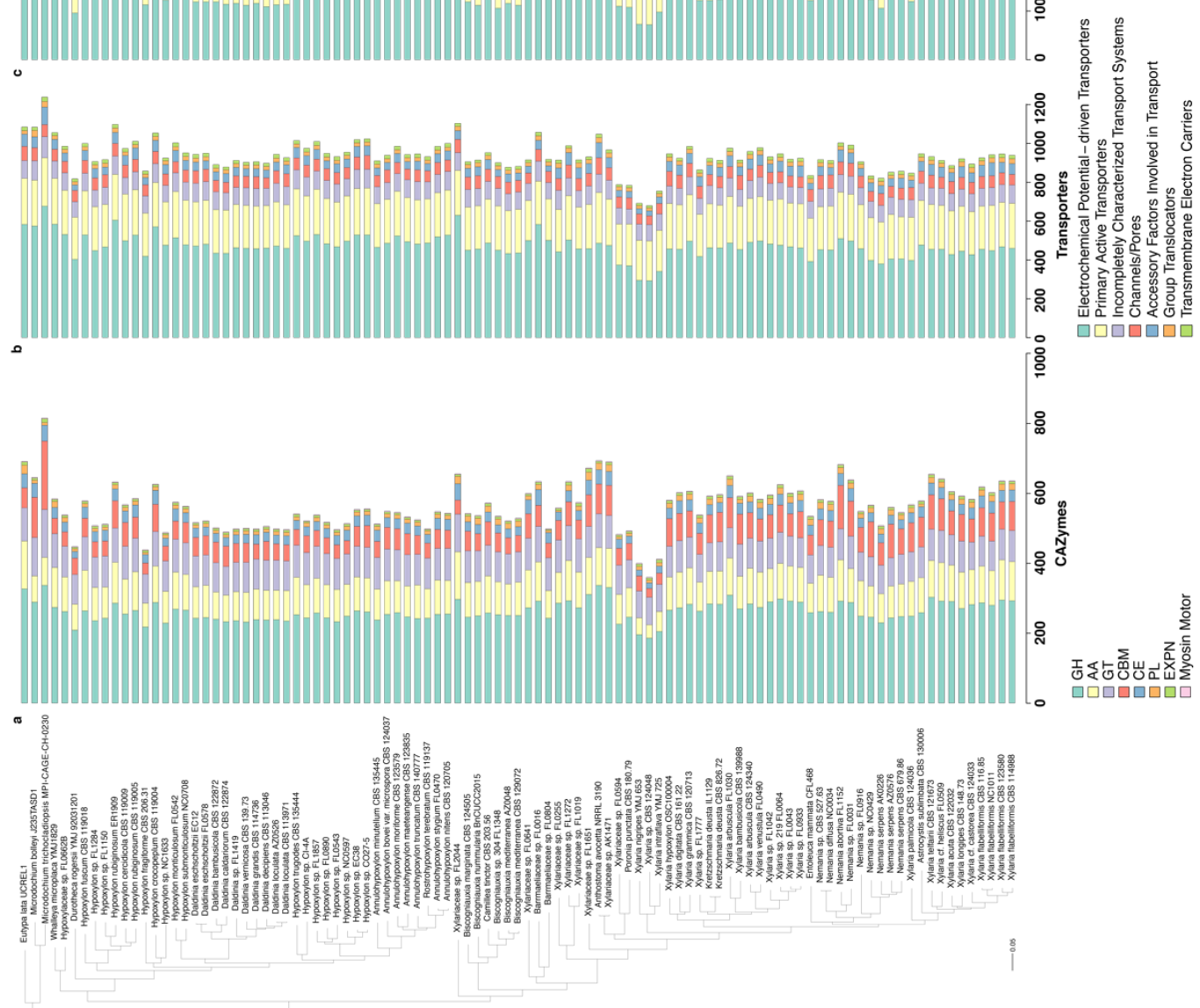

ڤั

प्रिप्र

Supplementary Figure 9. Relative abundance of functional gene categories across Xylariaceae s.l. and Hypoxylaceae. Phylogenomic tree and bar plot showing the abundance and identity of (a) carbohydrate-active enzymes (CAZyme); (b) peptidases and their inhibitors (MEROPs); (c) transporters (TCDB); (d) secreted proteins (SignalP); and (e) effectors (EffectorP). Colors refer to different classifications within each database (see legends). 


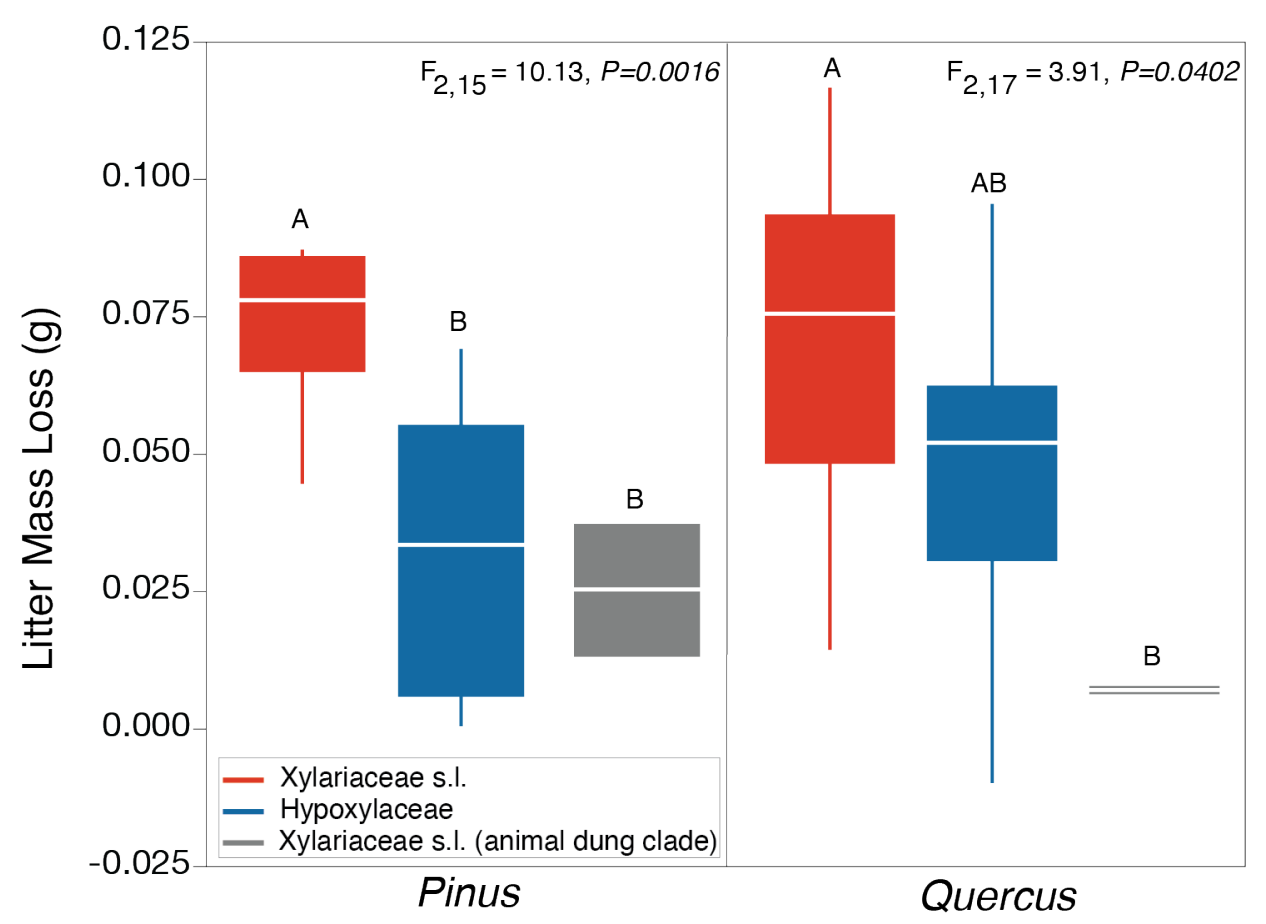

Supplementary Figure 10. Xylariaceae s.l. taxa demonstrate increased decomposition abilities (estimated via mass loss) on leaf litter compared to fungi with reduced genomes (i.e., Hypoxylaceae and animal dung Xylariaceae s.l. in the Poronia clade). Interquartile box plots showing median and interquartile range. We observed significant differences among means of each clade on both Pinus and Quercus leaves (ANOVA). Letters indicate significant differences after post-hoc Tukey's HSD. See Supplementary Table 1 for a list of isolates included in the mass loss experiment. 


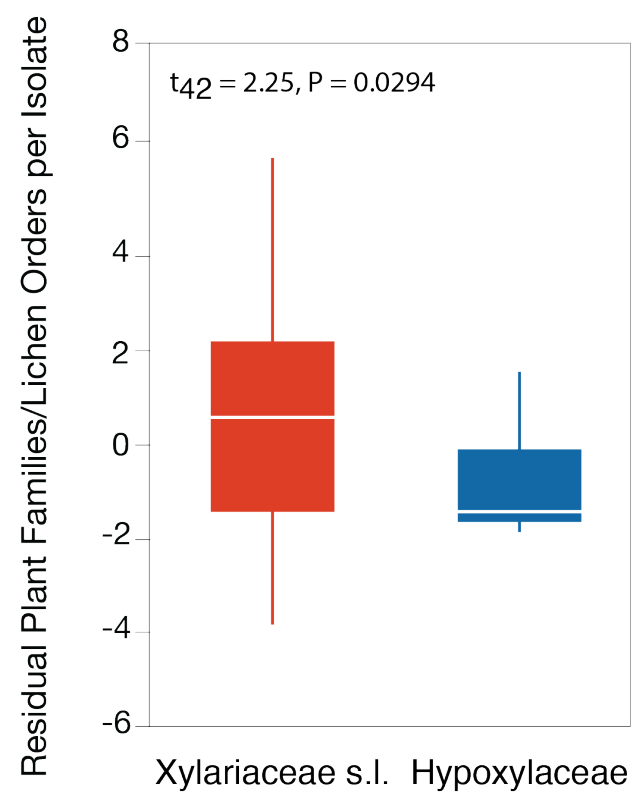

b

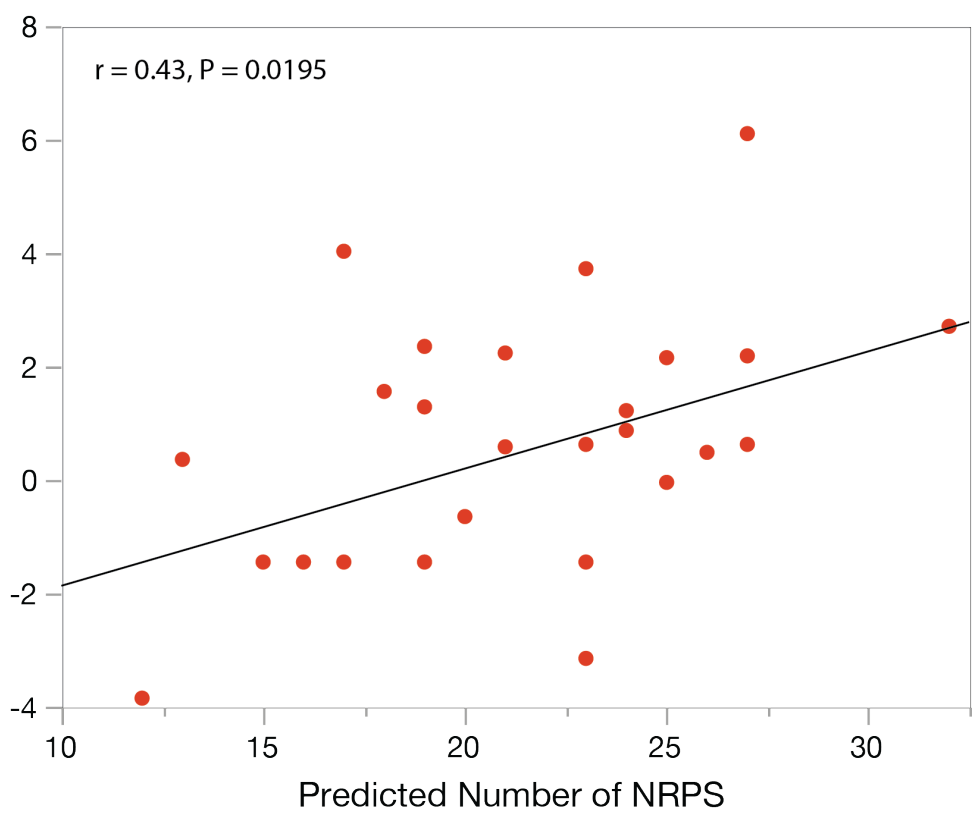

Supplementary Figure 11. Endophytes in the Xylariaceae s.l. clade have greater host breadth, which is correlated with an increase in NRPS SMGCs. (a) A quantile box plot showing the interquartile range and median of endophyte host breadth (measured as total number of plant families and lichen orders with which a fungal OTU was cultured; see ${ }^{7}$ ) as a function of major clade (color). T-test comparison of means illustrates greater host associations for Xylariaceae s.1. taxa ( $\mathrm{t}$-test, $\mathrm{t}_{42}=2.25, \mathrm{P}=$ 0.0294). A similar pattern was observed when only the number of plant families are compared (Wilcoxon: $\chi^{2}=4.14, \mathrm{P}=0.0413$ ), but not lichen orders (Wilcoxon: $\chi^{2}=1.77, \mathrm{P}=0.1834$ ). (b) Relationship of Xylariaceae s.l. endophyte host breadth and the number of SMGCs classified as NRPS. A similar pattern was observed when only the number of lichen orders was used to estimate host breadth (Pearson correlation: $\mathrm{r}=0.4944, \mathrm{P}=0.0064)$, but not for the number of plant families. Host breadth is also correlated with the number of HGT events $(r=0.43, \mathrm{P}=0.0193)$, but HGT events and SMGC content are not independent (Fig. 6). 


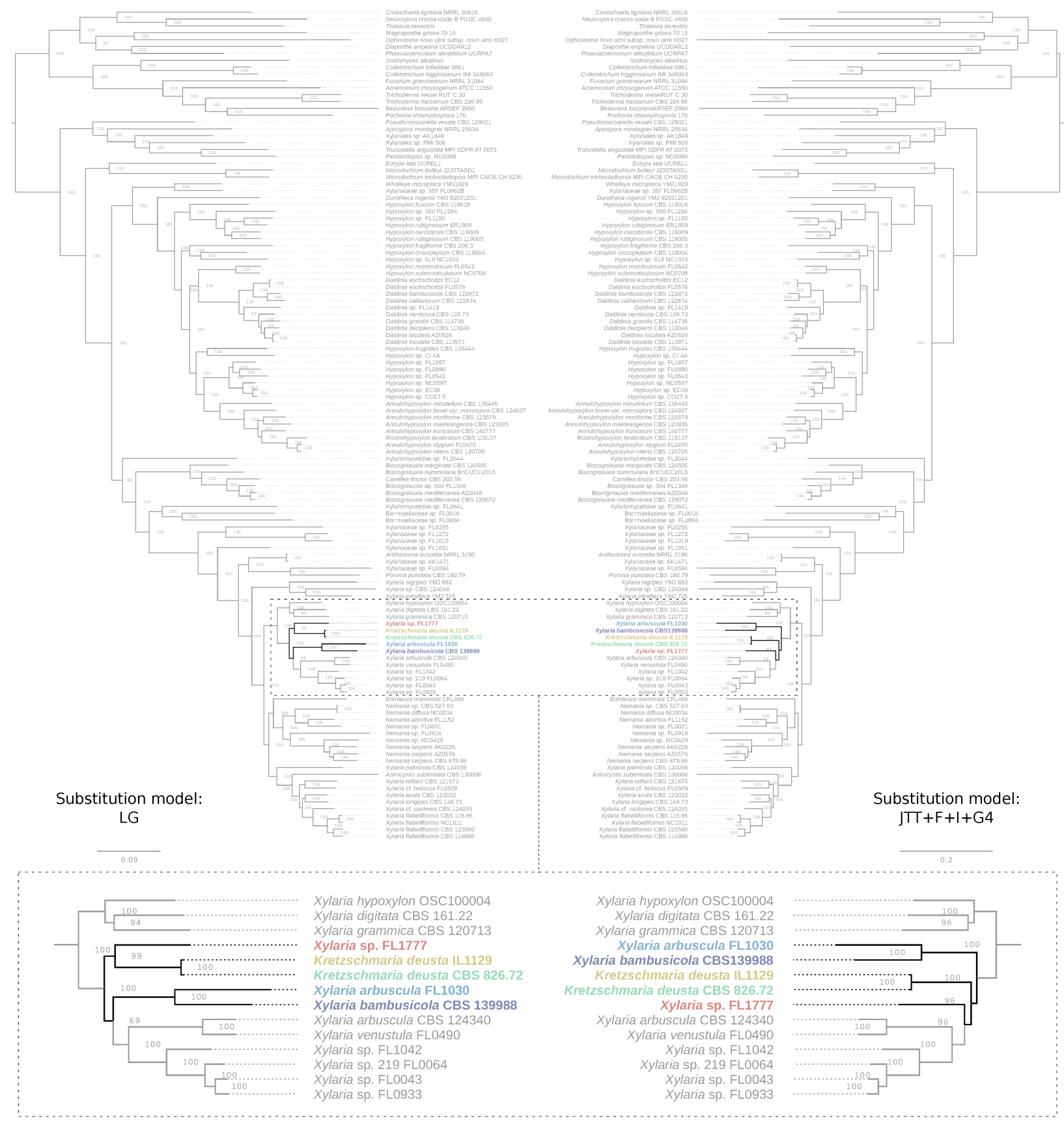

Supplementary Figure 12. Phylogenetic tree topology was robust to outgroup taxon selection, gene set, or model of evolution. (a) Phylogenetic tree from the concatenated analysis of 1,526 single-copy orthogroups performed in IQ-TREE with the LG model of evolution (i.e., analysis 1; see also Supplementary Fig. 1); (b) Phylogenetic tree resulting from analysis of the same orthogroups, but with the JTT + F + I + G4 model of evolution (i.e., analysis 2). Topological conflicts were rare; however, the placement of five Xylariaceae s.l. taxa differed slightly with different models of evolution. 


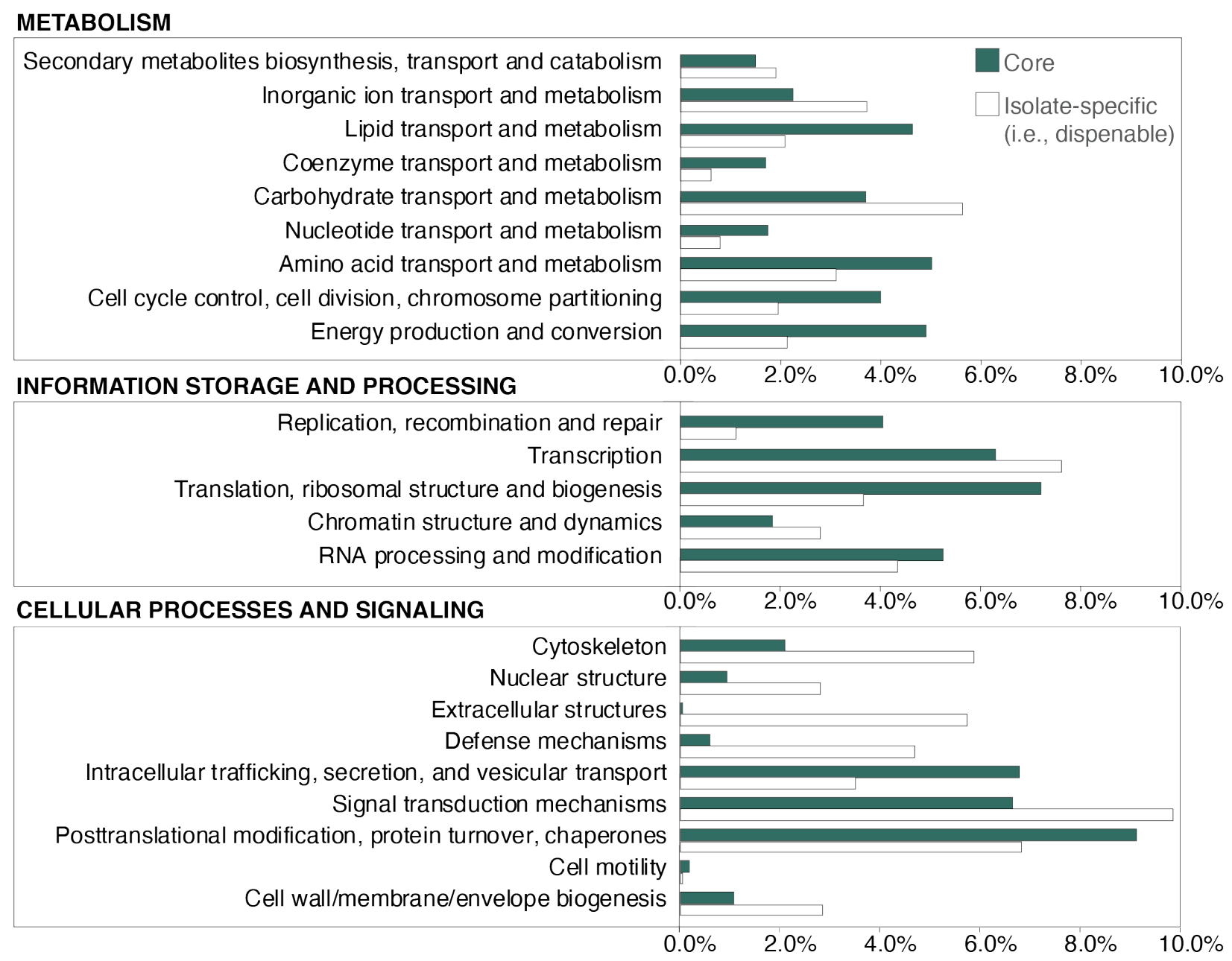

Supplementary Figure 13. Comparison of functional annotations for core and dispensable orthogroups. Bar graphs showing the relative abundance of different functional categories represented by "core" vs. "dispensable" orthogroups. Orthogroups were annotated with euKaryotic Orthologous Groups (KOGs; see Supplementary Table 7f). 


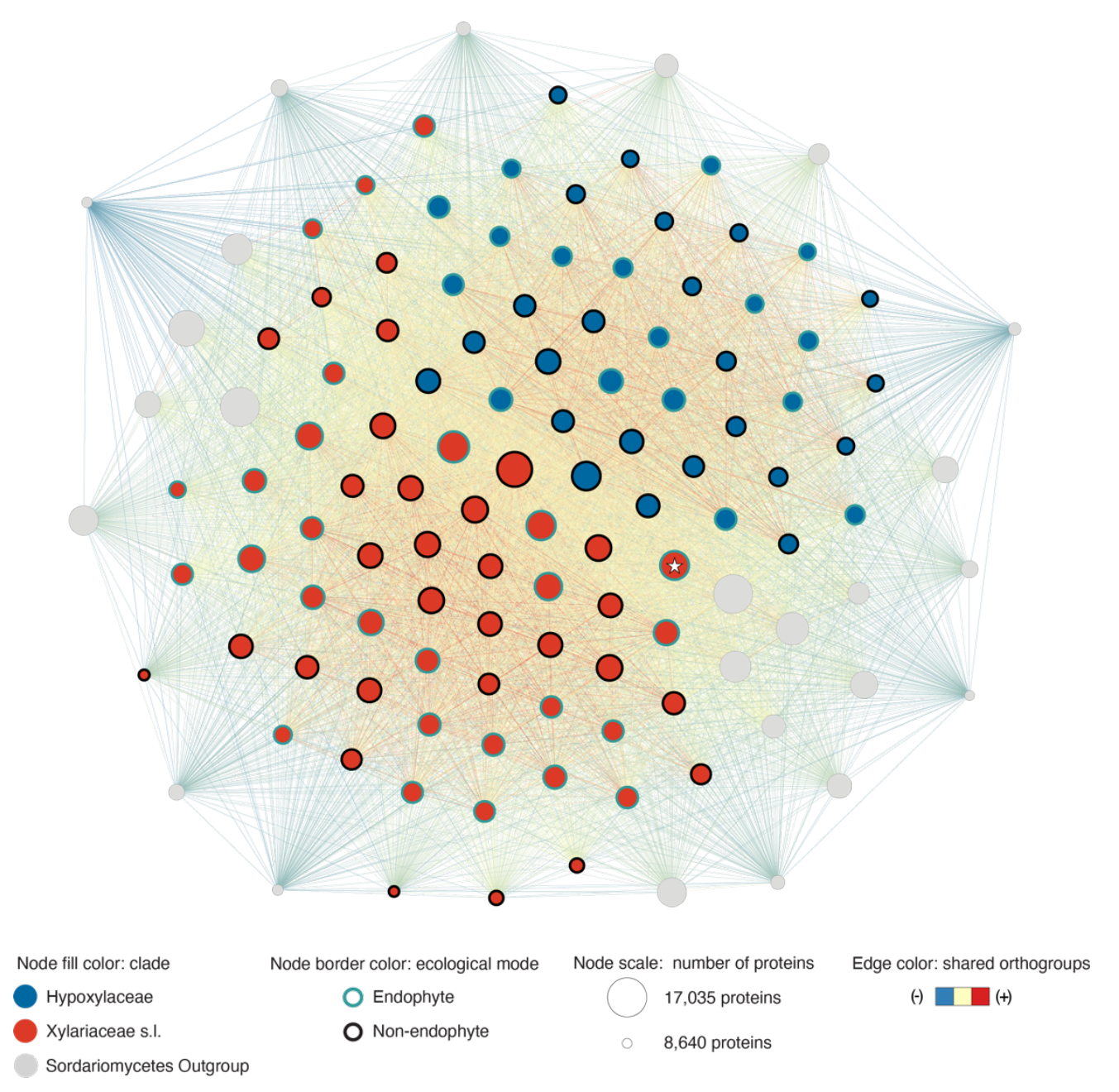

Supplementary Figure 14. Network analysis of individual proteomes illustrates the importance of major clade affiliation. Proteomes are represented by nodes, scaled by the count of proteins, colored by clade (fill) and ecological mode (border), and positioned by a force-directed layout algorithm. Edges between two nodes are weighted by the number of shared orthogroups. The node with a star represents Xylariaceae sp. FL2044. 


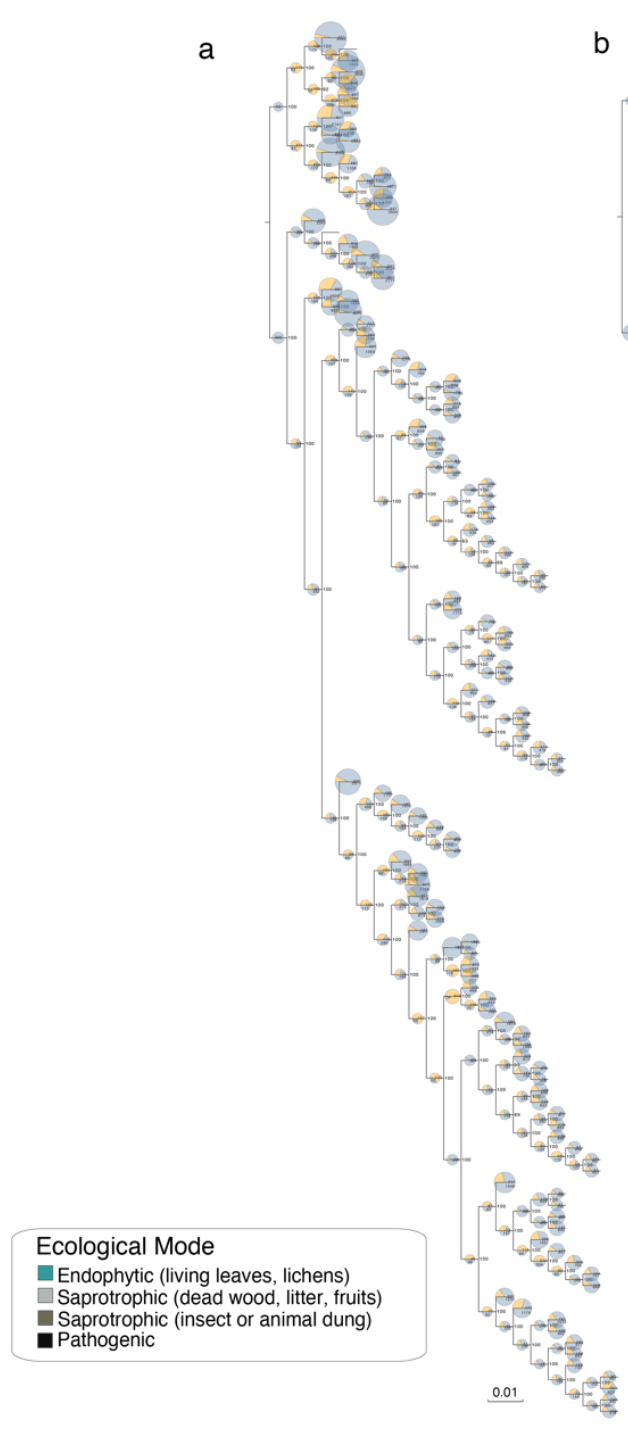

b

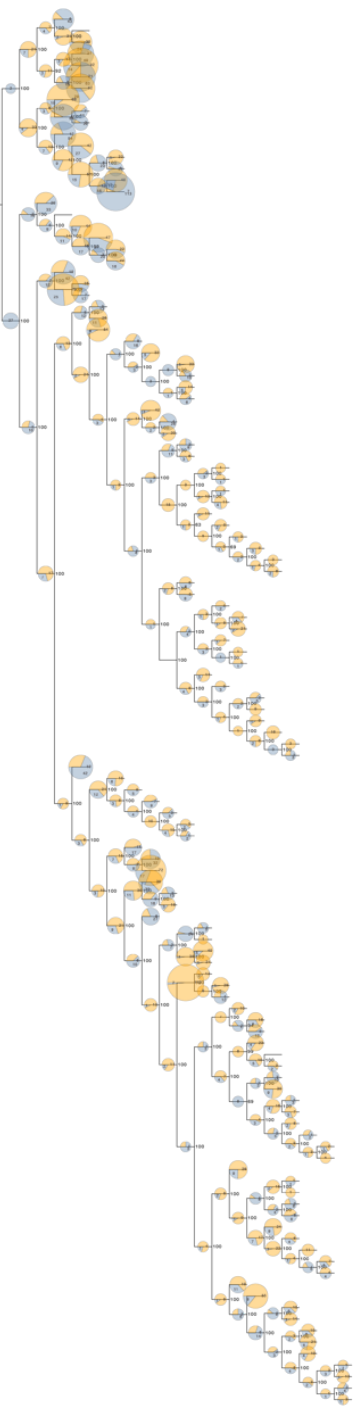

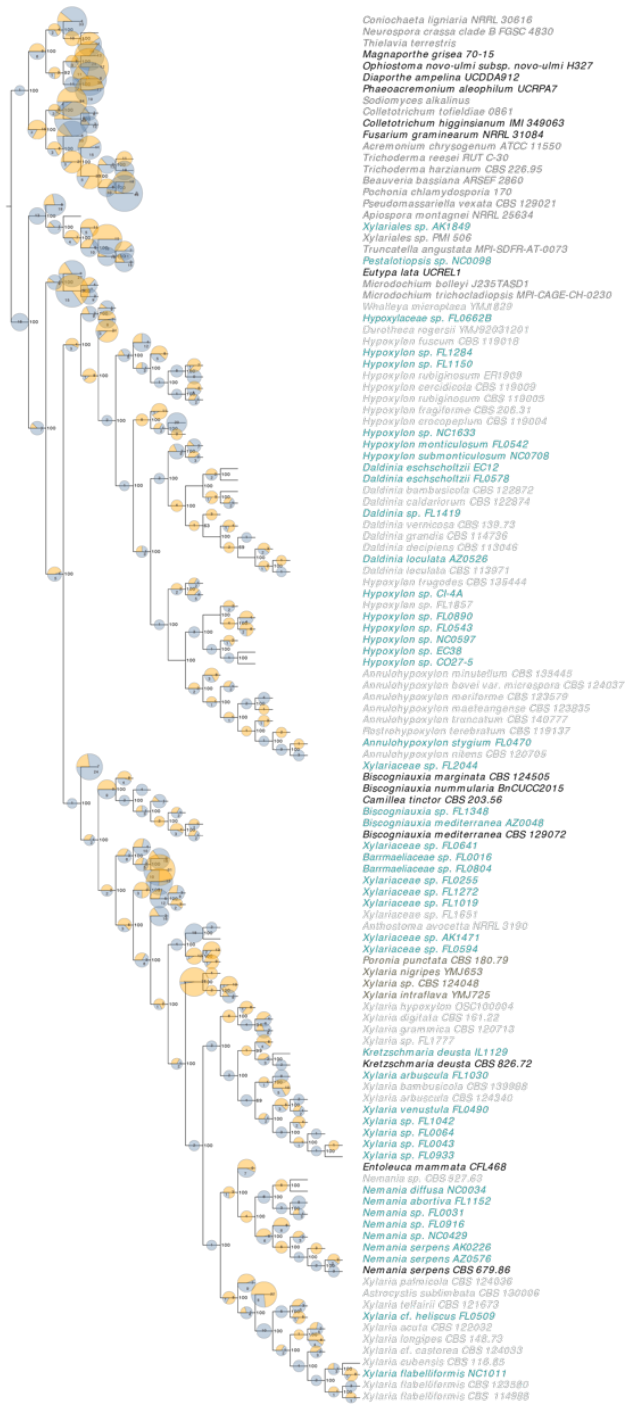

Supplementary Figure 15. Ancestral state reconstruction of orthogroups. The number of orthogroup gain (blue) and loss (orange) events for each node (inferred using the asymmetric Wagner parsimony method: gap penalty =1) are shown on the ML phylogenomic tree. The size of each pie chart is proportional to the total number of events inferred along the branch. Reconstructions were performed for (a) all orthogroups; (b) orthogroups annotated as CAZymes; and (c) orthogroups annotated as PCWDEs. Taxon names are colored by ecological mode (see legend). Predicted gains and losses were visualized on the phylogeny using EvolView ${ }^{133}$. 


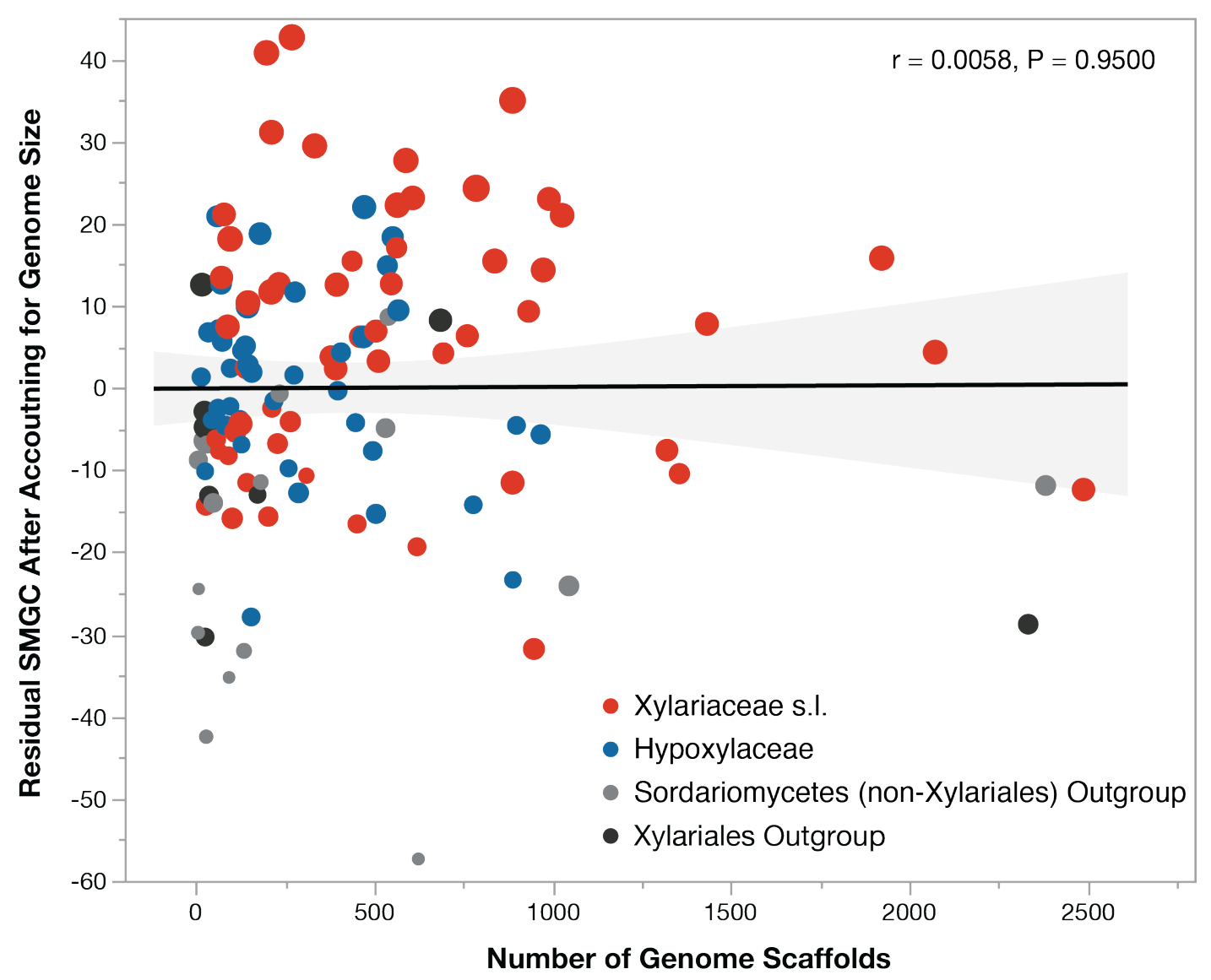

Supplementary Figure 16. The number of predicted SMGCs is not related to genome assembly. Relationship of predicted SMGC content (residuals after accounting for genome size) and the number of scaffolds for 121 genomes. Points are colored by clade and their size is proportional to the raw number of SMGC per genome (range 16-119). 